\title{
A MULTI-STANDARD SET-TOP BOX CHANNEL DECODER
}

by

Nirmal Sohi

A thesis submitted in conformity with the requirements

for the degree of Master of Applied Science

Graduate Department of Electrical and Computer Engineering

University of Toronto

c) Copyright by Nirmal Sohi 2000 
Acquialtions and Brbliographic Senvices

Moquinitione of sonvices bibliographiques

$\operatorname{ses}$ Weninoton Sincet Ovinm ON K1A ONA

3es, nu Womingen

Onimen ON Kila ONA

cand

cencera

The author has granted a nonexclusive licence allowing the National Library of Canada to reproduce, loan, distribute or sell copies of this thesis in microform, paper or electronic formats.

The author retains ownership of the copyright in this thesis. Neither the thesis nor substantial extracts from it may be printed or otherwise reproduced without the author's permission.
L'auteur a accordé une licence non exclusive permettant à la Bibliotheqque nationale du Canada de reproduire, prêter, distribuer ou vendre des copies de cette thèse sous la forme de microfiche/film, de reproduction sur papier ou sur format électronique.

L'auteur conserve la propriété du droit d'auteur qui protège cette thèse. $\mathrm{Ni}$ la thèse ni des extraits substantiels de celle-ci ne doivent être imprimés ou autrement reproduits sans son autorisation. 


\title{
A Multi-Standard Set-Top Box Channel Decoder
}

\author{
Nirmal Sohi \\ Master of Applied Science, 2000 \\ Department of Electrical and Computer Engineering \\ University of Toronto
}

\begin{abstract}
Digital transmission and recording technologies are revolutionizing the home-entertainment consumer electronics industry. Services such as Direct Broadcasting by Satellite (DBS) and Digital Versatile Disc (DVD) are rapidly gaining acceptance with consumers. It would be economical and convenient to use a single "set-top box" receiver for multiple services. This thesis describes the channel decoder design of a unified set-top box receiver. The standards for Digital Audio Broadcasting (DAB), Digital Video Broadcasting (DVB) and DVD are described first, to determine which receiver functions are amenable to being combined into reconfigurable VLSI soft-macro blocks. A survey of these standards indicates that a Viterbi decoder and/or a Reed-Solomon decoder for channel decoding are common functions that would benefit from co-integration. This thesis presents the synthesis-based design of a multistandard Viterbi decoder which has been fabricated in a $0.35 \mu \mathrm{m}$ CMOS process. A ReedSolomon decoder design which can operate with multiple standards is also presented.
\end{abstract}




\section{Acknowledgments}

This thesis would not bave been possible without the help of many people and organizations.

I would like to thank my advisor Professor Glenn Gulak for his support, encouragement and guidance.

I greatly appreciate the friendship and help of my colleagues in EECG. I am very fortunate to have had the opportunity to work with such intelligent and talented people. Thanks to Andy, Louis, Warren and especially Sean, for help with the CAD tools. Thanks to all of my other friends in LP392 and the EECG including Ali, Bach, Brent, Christian, Elias, Jason, Javad, Jeff, Ken, Lesley, Paul, Qiang, Sandy, Scott, Tooraj, Vaughn, Vince, and Yaska. Special thanks to Professor Frank Kschischang and Professor Emmanuel Boutillon for their useful discussions on the thesis work.

Financial assistance from NSERC, CSA, and chip fabrication support from CMC are greatly appreciated.

Most of all, I am deeply grateful to my family for their love, support and encouragement. 


\section{Table of Contents}

Chapter 1.

Introduction

1.1 Motivation

1.2 Objectives

1.3 Outline of Thesis

Chapter 2.

The Standards

2.1 DVB

2.1.1 Digital Video Broadcasting - Satellite (DVB-S)

2.1.2 Digital Video Broadcasting - Terrestrial (DVB-T)

2.1.3 Digital Video Broadcasting - Cable (DVB-C)

2.2 Digital Audio Broadcasting (DAB)

2.3 Digital Versatile Disc (DVD)

2.4 The Common Elements

2.5 Summary

\section{Chapter 3.}

Viterbi Decoder Design

3.1 The Viterbi Algorithm

3.2 Viterbi Decoder Structure $\quad 35$

3.2.1 Branch Metric Generator $\quad 35$

3.2.2 Add-Compare-Select Unit $\quad 42$

3.2.3 Survivor Memory Unit and Output Decision Device 44

3.2.4 Accomodating Different Standards $\quad 46$

3.3 Depuncturing Unit 48

3.4 Summary $\quad 52$

Chapter 4.

Reed-Solomon Decoder Design 53

4.1 Reed-Solomon Decoding $\quad 54$

4.2 Syndrome Calculation $\quad 57$

4.3 Modified Euclid's Algorithm $\quad 58$

4.4 Remaining Error Transform 61

4.5 Inverse Error Transform $\quad 62$

4.6 Accomodating Different Standards $\quad 63$

4.7 Summary $\quad 66$

Chapter 5.

Viterbi and Reed-Solomon Decoder Implementations 67

$\begin{array}{ll}5.1 \text { Viterbi Decoder } & 67\end{array}$

$\begin{array}{ll}\text { 5.1.1 Chip Architecture } & 67\end{array}$

5.1.2 Implementation Results $\quad 71$ 
5.1.3 Test

5.1.4 Critical Path $\quad 74$

5.2 Reed-Solomon Decoder 75

5.2.1 Architecture $\quad 75$

5.2.2 Implementation Results 76

$\begin{array}{ll}\text { 5.2.3 Test } & 78\end{array}$

5.3 De-interleaver $\quad 79$

$\begin{array}{ll}5.4 \text { Summary } & 80\end{array}$

Chapter 6.

Conclusions

81

6.1 Summary and Conclusions

$\begin{array}{ll}6.2 \text { Contributions } & 82\end{array}$

6.3 Future Work $\quad 82$

References

84

Appendix A

DVB-T Specification

89

Appendix B

Galois Dield Primer

93

Appendix C

Time Domain Reed-Solomon Decoding

98

Appendix D

Hardware for Erasure RS Decoding

101

Appendix $\mathbf{E}$

Optimixing the ACS Arithmetic Units

104 


\section{List of Tables}

Table 2.1: DVB Punctured code definition [1] 11

Table 2.2: Error performance requirements [1] 12

Table 2.3: Summary of system parameters $\quad 26$

Table 4.1: RS parameters for DVB, DVD 64

$\begin{array}{ll}\text { Table 5.1: Chip characteristics } & 72\end{array}$

Table 5.2: I/O Pin Descriptions

Table 5.3: Estimated area of RS decoder components using parallel GF arithmetic units 78

Table A.1: $\mathrm{GF}\left(2^{4}\right)$ elements generated from $\alpha^{4}+\alpha+1=0$ 


\section{List of Figures}

Fig. 2.1: MPEG-2 transport multiplex creation [1] 6

Fig. 2.2: Structure of packets produced by MPEG-2 transport MUX 7

Fig. 2.3: Transmission side of DVB-S

$\begin{array}{ll}\text { Fig. 2.4: PRBS circuit } & 8\end{array}$

Fig. 2.5: Randomized transport packets $\quad 8$

Fig. 2.6: Reed-Solomon RS $(204,188)$ error protection packet 9

Fig. 2.7: Byte interleaver and de-interleaver defined in DVB standards [1] 9

Fig. 2.8: Interleaved frame structure 9

$\begin{array}{ll}\text { Fig. 2.9: Convolutional encoder } & 10\end{array}$

Fig. 2.10: QPSK constellation [1] 11

Fig. 2.11: DVB-S receiver block diagram 12

Fig. 2.12: Transmission side of DVB-T [2] 15

Fig. 2.13: Inner coding/interleaving 16

$\begin{array}{ll}\text { Fig. 2.14: DVBIRD receiver for DVB-T } & 17\end{array}$

$\begin{array}{ll}\text { Fig. 2.15: DVB-C receiver } & 18\end{array}$

Fig. 2.16: DAB Transmission Frame [29] 19

Fig. 2.17: DAB receiver system $\quad 20$

Fig. 2.18: Generation of DVD readout signal 22

Fig. 2.19: Gaussian impulse response with $\mathrm{t}_{0}=150 \mathrm{~ns}, \mathrm{~T}=37.04 \mathrm{~ns}$

Fig. 2.20: NRZI waveform and corresponding DVD readout waveform 23

Fig. 2.21: Data extraction using a slicer 24

Fig. 2.22: Viterbi-detector based readout system 25

Fig. 2.23: Channel decoding common elements ( $\pi^{-1}$ represents a de-interleaver) 27

Fig. 3.1: Shift register model of a Markov process observed in memoryless noise 30

Fig. 3.2: Trellis representation of Markov process 31

Fig. 3.3: Convolutional encoder for DVB 34

Fig. 3.4: DVD ISI shift-register process 34

Fig. 3.5: Viterbi Decoder structure $\quad 35$

Fig. 3.6: Convolutional encoder and corresponding trellis 36 
Fig. 3.7: ISI shift-register process and corresponding trellis

Fig. 3.8: BER performance for different branch metrics 39

Fig. 3.9: BER performance for different quantization levels $\quad 40$

Fig. 3.10: Quantized Constellation 41

Fig. 3.11: Flexible branch metric generator 44

Fig. 3.12: ACS cell for state $r$

Fig. 3.13: ACS cell for state $r$ using a 2's complement subtractor to generate the decision bit 43

Fig. 3.14: One stage of the SMU - $\delta$ of these would be cascaded to form the SMU 44

Fig. 3.15: Rate 7/8 DVB-S BER performance for various merge depths 46

Fig. 3.16: EFMPlus constrained states and transitions $\quad 47$

Fig. 3.17: DVB-S code rate selection and depuncturing 49

Fig. 3.18: Depuncturing Unit: high-level hardware organization 51

Fig. 4.1: Error correcting frequency-domain Reed-Solomon decoder using the modified Euclid algorithm 55

Fig. 4.2: Syndrome calculation $\quad 57$

Fig. 4.3: Modified Euclid's algorithm block for the computation of one recursion 61

Fig. 4.4: Calculation of remaining error transform 62

Fig. 4.5: Inverse Transform calculation $\quad 62$

Fig. 4.6: DVD RS-PC frame structure [49] 64

Fig. 4.7: Errors and erasure RS decoder 65

$\begin{array}{ll}\text { Fig. 5.1: Overall chip architecture } & 67\end{array}$

Fig. 5.2: DVB and DAB branch metric calculation for cell number iqjr 68

Fig. 5.3: Reading in Branch Metric Comparison (BMC) values 69

Fig. 5.4: ACS sub-unit $\quad 70$

Fig. 5.5: ODD cell 1, row $1 \quad 70$

Fig. 5.6: ODD high level structure $\quad 71$

Fig. 5.7: Viterbi decoder chip layout $\quad 72$

Fig. 5.8: RS-PC decoder structure $\quad 76$

Fig. 5.9: Basic de-interleaver structure $\quad 79$

Fig. A.1: 16-QAM inner interleaver [2] 90 
Fig. A.2: Uniform 16-QAM constellation [2]

Fig. A.3: OFDM frame structure [2]

Fig. C.1: Time domain decoder structure [46] 99

Fig. C.2: Polynomial evaluation for s [46] 100

Fig. D.1: $\alpha^{k}$ generation $\quad 102$

Fig. D.2: Polynomial expansion 2 [45] 103

Fig. D.3: Polynomial expansion 1 [45] 103

Fig. E.1: 12-bit ACS adder with ripple-carry chain as part of 16 ns critical path 105

Fig. E.2: 12-bit ACS adder with critical path reduced to $\sim$ ns 106 


\section{Acronyms}

ACS

Add-Compare-Select

AMD

Analog to Digital

ADC

Analog to Digital Converter

AWGN

Additive White Gaussian Noise

BB

Baseband

BER

Bit Error Rate

BMC

Branch Metric Comparison

BMG

Branch Metric Generator

CSI

Channel State Information

DAB

Digital Audio Broadcasting

DBS

Direct Broadcasting by Satellite

DQPSK Differential Quadrature Phase Shift Keying

DSS DirecTV Digital Satellite System

DTH Direct-To-Home

DU Depuncturing Unit

DVB Digital Video Broadcasting

DVB-C Digital Video Broadcasting-Cable

DVB-S Digital Video Broadcasting-Satellite

DVB-T Digital Video Broadcasting-Terrestrial

DVBIRD Digital Video Broadcasting Integrated Receiver Decoder

DVD Digital Versatile Disc

EEP Equal Error Protection

EFM Eight-to-Fourteen Modulation

EFMPlus Eight-to-Fourteen Modulation Plus

FDD Frequency-Domain Decoder

FIB Fast Information Block

FIC Fast Information Channel

FFT Fast Fourier Transform 


\begin{tabular}{ll} 
GF & Galois Field \\
IC & Integrated Circuit \\
IF & Intermediate Frequency \\
VO & InputOutput \\
ISI & Intersymbol Interference \\
MB & Mega-byte \\
Mbps & Mega-bits per second \\
MCI & Multiplex Configuration Information \\
MEA & Modified Euclid's Algorithm \\
MLSD & Maximum-Likelihood Sequence Detection \\
MPEG & Moving Pictures Expert Group \\
MSB & Most Significant Bit \\
MSC & Main Service Channel \\
MUX & Multiplexer \\
NRZI & Non-Return to Zero Inverted \\
ODD & Output Decision Device \\
OFDM & Orthogonal Frequency Division Multiplexing \\
PCS & Personal Communication Services \\
PRBS & Pseudo-Random Binary Sequence \\
PS & Pseudo-Syndrome \\
QAM & Quadrature Amplitude Modulation \\
QEF & Quasi-Error Free \\
QPSK & Quadrature Phase Shift Keying \\
RF & Radio Frequency \\
RLL & Run-Length Limited \\
RS & Reed-Solomon \\
RS-PC & Reed-Solomon Product Code \\
SC & Synchronization Channel \\
SDTV & Standard Definition Television \\
SFN & Single Frequency Network \\
SNR & Signal-to-Noise Ratio \\
\hline
\end{tabular}


SOVA Soft Output Viterbi Algorithm

STB Set-Top Box

TDD Time Domain Decoder

TPS Transmission Parameter Signalling

UEP Unequal Error Protection

UHF Ultra-High Frequency

VHF Very-High Frequency

VA Viterbi Algorithm

VD Viterbi Decoder

VHDL VHSIC Hardware Description Language

VHSIC Very High Speed Integrated Circuit 


\section{Symbols}

Viterbi Decoder:

$\delta \quad$ Survivor length (also called "merge depth")

$\lambda \quad$ Branch metric

$v \quad$ Number of memory elements in a shift-register process

$\pi \quad$ Interleaver

$\pi^{-1} \quad$ De-interleaver

$\mathrm{E}_{\mathrm{b}} / \mathrm{N}_{0} \quad$ Energy per bit divided by one-sided noise power spectral density

$h_{j} \quad$ Impulse response samples

$s_{k} \quad$ A state at time $k$

s State sequence

$\hat{s}\left(s_{k}\right) \quad$ Survivor sequence of state $s_{k}$

$u_{k} \quad$ Information bit

$y_{k} \quad$ Input to a memoryless channel

$z_{k} \quad$ Output of a memoryless channel

$D_{k} \quad$ Decision bit of state $k$

DP Depuncturing state

$L\left(s_{k}\right) \quad$ Survivor length of state $s_{k}$

I An input to the QPSK modulator and input to de-puncturing unit

Q An input to the QPSK modulator and input to de-puncturing unit

$X \quad$ First output for the rate $1 / 2$ DVB encoder

Y Second output for the rate 1/2 DVB encoder 
Reed-Solomon Decoder:

$\begin{array}{ll}N & \text { Reed-Solomon codeword length } \\ I & \text { Number of information symbols in a Reed-Solomon codeword } \\ t & \text { Error-correcting capability of a Reed-Solomon code } \\ r_{n} & \text { symbols of received codeword } \\ S_{k} & k^{\prime} \text { th syndrome coefficient } \\ T(x) & \text { Forney syndrome } \\ \Lambda(x) & \text { Erasure locator polynomial } \\ \sigma_{k} & k^{\prime} \text { th error locator coefficient } \\ E_{k} & k^{\prime} \text { 'th error transform coefficient } \\ e_{k} & k^{\prime} \text { th error pattern coefficient }\end{array}$




\section{Chapter 1. Introduction}

\subsection{Motivation}

Recently developed digital transmission and recording systems are gradually taking the place of older analog broadcasting and recording systems. The growing popularity of digital television broadcasting and Digital Versatile Disc (DVD) illustrate this trend. Consumers generally require a separate device to use each of these digital services, each device processing a different high speed bit-stream. An area currently of great interest is "convergence" of some of these devices into a single integrated receiver. If different services use the same receiver, economies of scale can result in more devices being sold and a lower device cost. This would be beneficial to both service providers and consumers.

The term "information furnace" is used here to represent an integrated interface between the user and various services supported by high speed bit streams provided to the home. The development of such a unified home appliance is an ongoing and evolutionary process. There are integration issues on many levels, not all technical in nature. At the system integration level, determining which functions to perform in hardware and which to perform in software represents a trade-off between performance and flexibility. At the highest level, it is not known what combination of features consumers will value. In short, the vast scope of digital-convergence issues results in many different design approaches being applied to different applications. Some examples of this are:

- Sharewave Inc. is promoting the concept of multimedia-capable wireless home networking. This could be used to broadcast signals from, for example, a DVD player or Direct Broadcast Satellite (DBS) receiver to various display devices within the home [10].

- In developing what they call "dynamically reconfigurable logic", MorphICs Technology Inc. is focussing on the high level of performance and reconfigurability required by multi-standard wireless Personal Communication System (PCS) platforms [11]. 
- To be released in 2000, the Sony Playstation 2 game console contains a DVD player [12].

- Broadcom has developed a single-chip digital satellite receiver for Digital Video Broadcasting by Satellite (DVB-S), the DirecTV Digital Satellite System (DSS) and Digicipher-II compliant DBS systems [19].

Home entertainment is a major application of emerging digital services. Most users of new digital video services already own the most expensive system component: the display device (television). The focus here will be on combining the reception of such digital services in a so-called "set-top box", which is typically connected to a television set. The term "set-top box" is used here to represent an information furnace which connects to a television, an audio system and/or a personal computer.

Details of most digital services are specified in standards which precisely define required system characteristics. Examination of receiver functions for certain digital standards reveals that much of their underlying signal processing is similar. The description of some services which are good candidates for convergence in a home-based receiver are now described.

The Digital Video Broadcasting (DVB) Project includes over 220 organisations (e.g., broadcasters, manufacturers, regulatory bodies) in more than 30 countries. Its goal is the design of a family of standards for digital television delivery. Many DVB standards have been successfully implemented worldwide. The most widely used standard is DVB transmission via Satellite (DVB-S). Other DVB standards being adopted are the cable specification (DVB-C), and the terrestrial transmission standard (DVB-T) [5]. Although other Direct Broadcasting by Satellite (DBS) standards are used (e.g., DirecTV), their standards are proprietary and are not considered in this thesis. However, their specifications are similar to DVB-S, and these standards have already been combined in one receiver implementation [19].

Digital Audio Broadcasting (DAB) based on the Eureka 147 standard [4] provides specifications for digital radio transmission. DAB services are already in operation in some European countries 
(e.g., U.K., Germany, France), and DAB has been adopted for use in many countries, including Canada. The United States is planning a different digital audio broadcasting standard, In-Band On-Channel (IBOC) DAB [7] because of bandwidth limitations and licensing issues. DAB provides near CD-quality sound through the use of MPEG-2 audio coding. It also enables data transmission, allowing receivers to display information related to the sound broadcast [6]. DAB has provision for a low bit-rate upstream link for two-way services.

The Digital Versatile Disc (DVD) is becoming widely accepted as a digital video storage medium. Read-only 4.7 Gbyte DVD's are used to distribute computer software, but the most popular use of DVD's has been for movie distribution [8].

Because DVD players and DVB receivers are to be attached to a television, these two devices are amenable to being combined. Presently, DAB receivers are relatively large, making DAB reception suitable for stationary home-stereo systems. This implies the possibility of combining DVB, DVD and DAB into a single receiver for home-entertainment systems. The MPEG-2 standard ([34], [35], [36]) for source encoding of digital signals (e.g., audio, video) underlies most new digital services, including DVB, DVD and DAB. Since the MPEG-2 decoder is one of the receiver's most complex VLSI cores, it would be desirable to use a single MPEG-2 decoder for multiple applications.

\subsection{Objectives}

The focus of this thesis is on the "channel decoding" components ("data detection" components for DVD) required for reception of certain home-entertainment digital services. The term channel decoding refers to that part of the receiver which mitigates errors introduced by the transmission channel. These services are defined by the following standards:

- DAB (Digital Audio Broadcasting)

- DVB-S (Digital Video Broadcasting - Satellite)

- DVB-T (Digital Video Broadcasting - Terrestrial) 
- DVB-C (Digital Video Broadcasting - Cable)

- DVD (Digital Versatile Disc)

Most DVB channel decoding requirements are the same. The system description and receiver requirements for these standards is explained in Chapter 2. It will be shown in Chapter 2 that a Viterbi decoder (VD) and Reed-Solomon (RS) decoder are used in most of the standards. Since the VD design requires internal changes to its structure in order to accommodate different standards, most of the design effort is devoted to the VD. However, since the same core RS decoder can be used for the standards, less detail is given for its design. Since DVB-S and DVD are the two most widely adopted digital services in question, performance simulations of DVB-S and DVD are principally used in constraining the VD design. The VD design and its VLSI implementation results are presented. For the RS decoder, a potential architecture is proposed, and its components are synthesized to obtain performance and cost information.

Since time-to-market is extremely important for new telecommunications IC products in general and consumer products in particular, it is becoming necessary to adopt a new paradigm of design based on soft-cores, or Intellectual Property (IP) reuse. This new methodology involves reuse of existing design blocks wherever possible, raising the level of design entry [9]. This shift in design style is apparent in the products of companies (eg. VLSI Technology [13], 4i2i Corp. [14]) which now provide soft-cores, which can significantly reduce design time for telecommunications products. With the approach of IP reuse in mind, custom layout is avoided in this thesis, with all components specified in VHDL. The components are parameterized to facilitate changing of certain hardware system parameters.

\subsection{Outline of Thesis}

A description of the standards under consideration is given in Chapter 2, which concludes with a justification of the thesis objectives. The VD design is presented in Chapter 3, and the RS decoder design is given in Chapter 4. Chapter 5 presents implementation results: the VD chip specifications are presented, along with specifications of synthesizable RS decoder components. Chapter 6 concludes the thesis and provides some future directions. 


\section{Chapter 2. The Standards}

This chapter briefly describes the transmission and reception systems for the DVB-S, DVB-T, DVB-C, DAB and DVD standards. The purpose here is not to reproduce the standards, but to describe a subset of their information which is sufficient to accomplish the thesis objectives: identifying the channel decoding algorithms common to each of the standards and proposing a reconfigurable hardware architecture that can satisfy the performance requirements while minimizing cost. The standards themselves typically only define the transmitter structure and data formats. In some cases they explicitly define some receiver characteristics, but hardware specification of the receivers is kept to a minimum. Some of the high-level receiver architectures presented in this chapter are based on recommendations in the standards, and some are adapted from actual receiver implementations. Design details of certain components such as the depuncturing unit, Viterbi decoder (VD) and Reed-Solomon (RS) decoder are presented in later chapters.

\subsection{DVB}

As described earlier, the Digital Video Broadcasting (DVB-S, DVB-T and DVB-C) standards have similar channel decoding operations. It has been estimated that the complexity of a DVB-T receiver is $5 \%$ to $25 \%$ more complex than a DVB-C receiver, which in turn is $5 \%$ more complex than a DVB-S receiver [23]. This section briefly describes the receiver signal processing components from the physical interface to the MPEG-2 demultiplexer.

\subsubsection{Digital Video Broadcasting - Satellite (DVB-S)}

DVB-S is the most widely adopted of the DVB family of standards, and is in use worldwide. In terms of Direct-To-Home (DTH) satellite services, DVB-S is the most widely used standard [5].

In DTH satellite services, protection of the satellite signal against noise and interference is the main design objective. This is because DTH satellite services are more affected by power 
limitations than by spectrum efficiency requirements. Satellite power is limited because it is derived from solar energy or expendable resources. The use of Quadrature Phase Shift Keying (QPSK) modulation and a concatenated coding system (convolutional and RS codes) results in power efficiency and a reasonable spectrum efficiency [1].

The RF frequency range for DVB-S is $10.70-12.75 \mathrm{GHz}$ [16]. The signal bandwidth determines the maximum bit rate which can be transmitted, and is a characteristic of the satellite transponder used. The signal bandwidth and maximum bit-rate are not fixed in the DVB-S standard, although it gives examples of bit-rates and their corresponding transponder bandwidths. The largest bitrate listed is 42.2 Msymbols/sec (84.4 Mbps for QPSK) in a $54 \mathrm{MHz}(-3 \mathrm{~dB})$ bandwidth transponder (the DVB-S standard [1] uses a (Bandwidth)/(Symbol Rate) factor of 1.28 for this calculation). A practical transponder bandwidth for DVB-S signals is $33 \mathrm{MHz}$ [16], [18]. This corresponds to a maximum gross bit rate of $51.60 \mathrm{Mbps}$ [16], which is adequate to provide five standard-definition TV (SDTV) programs [18].

Before describing the receiver, the DVB-S transmitter functions are briefly described. The DVB systems use MPEG-2 for source-encoding. The MPEG-2 standard is described in [34], [35] and [36]. Fig. 2.1 shows how the MPEG-2 transport multiplex is created by source coding and multiplexing. Each program consists of different media streams. These programs are multiplexed into a single stream of transport multiplex packets.

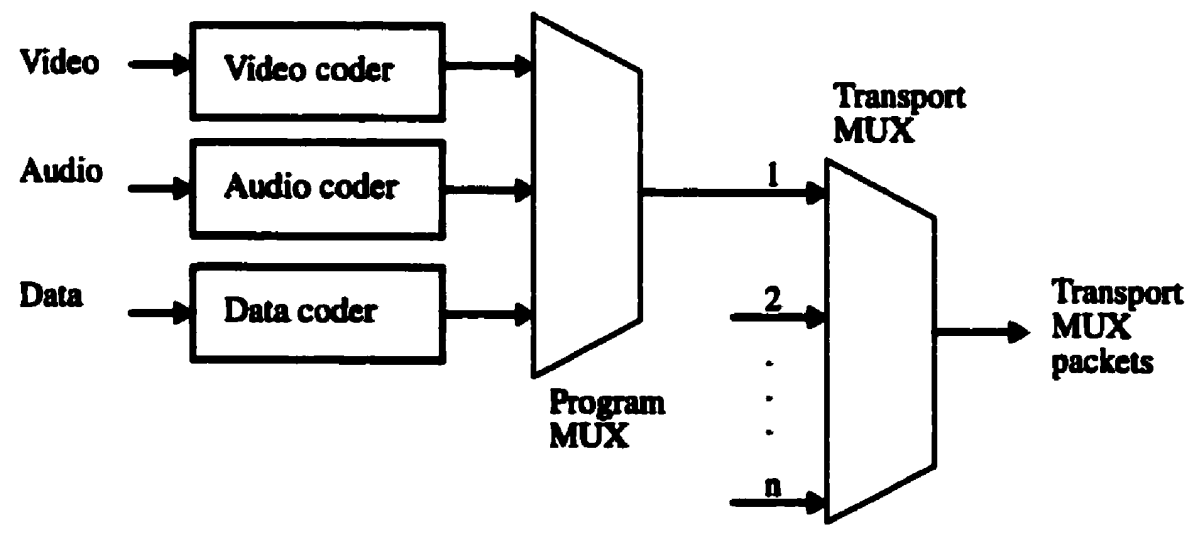

Fig. 2.1: MPEG-2 transport multiplex creation [1] 
The MPEG-2 transport multiplex packet length is 188 bytes, including 1 sync byte (01000111). Processing at the transmitter starts with the MSB of the sync byte (i.e., 0 ). The packet structure of the MPEG-2 transport multiplexer (MUX) output is shown in Fig. 2.2.

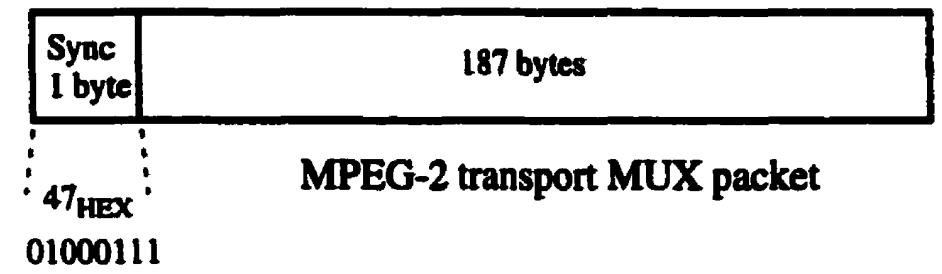

Fig. 2.2: Structure of packets produced by MPEG-2 transport MUX

Fig. 2.3 shows how the MPEG-2 transport MUX output is processed in the transmitter. Note that the transmitter uses a Reed-Solomon encoder and a convolutional encoder. This is referred to as a "concatenated coding system", with the RS code called the "outer code" and the convolutional code referred to as the "inner code".

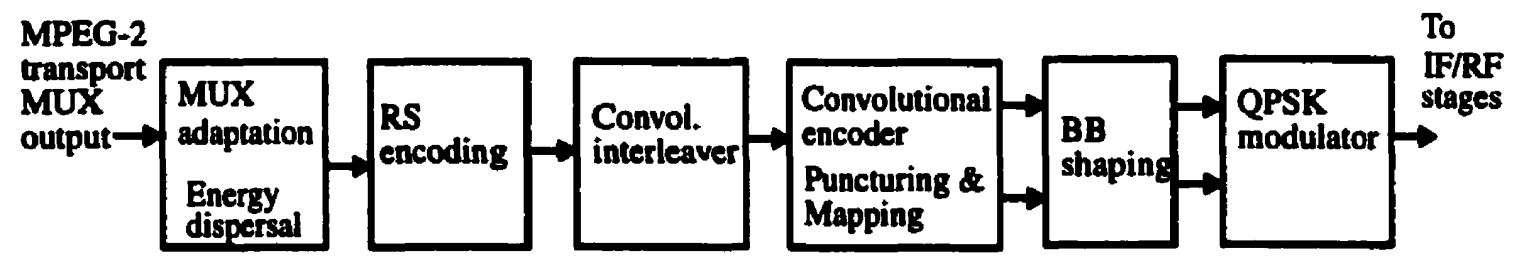

Fig. 2.3: Transmission side of DVB-S

In order to provide initialization signals for the descrambler, every eighth sync byte is bit-wise

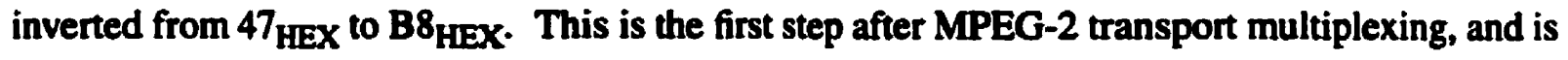
called "transport multiplex adaptation".

The next step is to randomize the data to ensure adequate binary transitions (i.e., energy dispersal). Fig. 2.4 shows the pseudo-random binary sequence (PRBS) generator circuit used to randomize the data once it has undergone transport MUX adaptation. Every eight transport packets, the sequence " 100101010000000 " is loaded into the shift register of Fig. 2.4. The polynomial for this PRBS is $1+X^{14}+X^{15}$. 


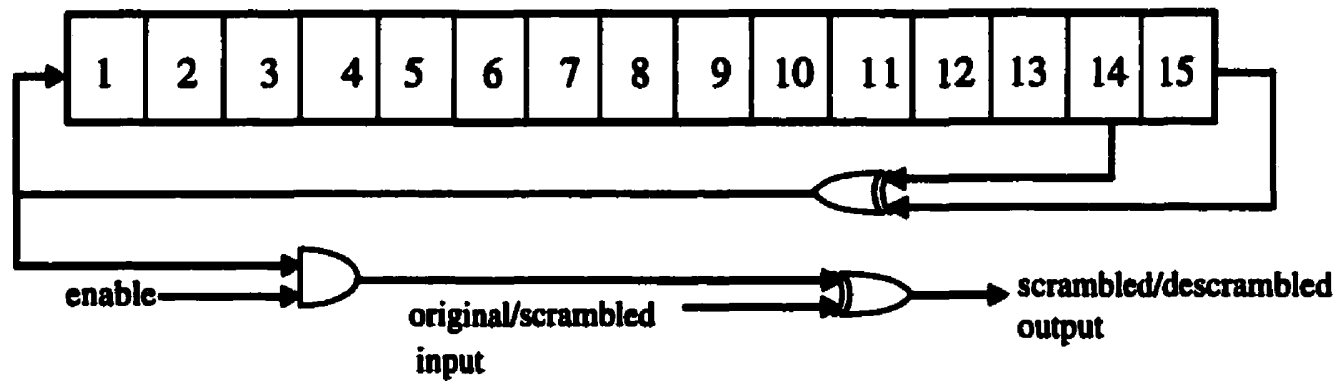

Fig. 2.4: PRBS circuit

The first bit output by the PRBS generator is applied to the first bit (MSB) of the first byte following the inverted MPEG-2 sync byte ( B8 $\left._{\mathrm{HEX}}\right)$. The enable signal in Fig. 2.4 is set to 0 only for the following 7 sync bytes. In this way, the PRBS generation continues, but the output is disabled, leaving the sync bytes unrandomized. As illustrated in Fig. 2.5, the period of the PRBS is 1503 bytes.

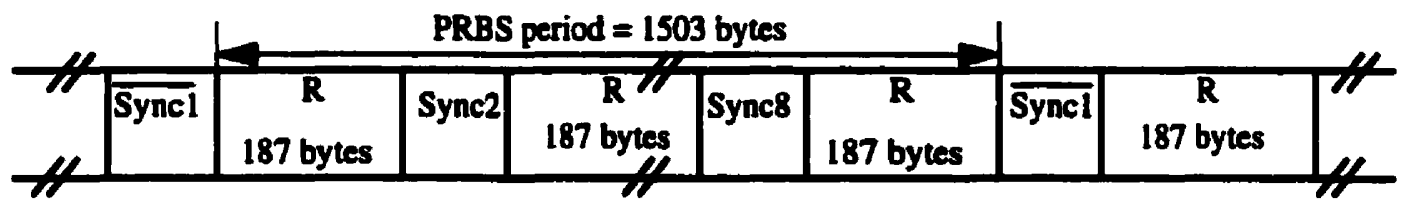

(Sync bytes and randomized sequence R)

Fig. 2.5: Randomized transport packets

Following the randomization (energy dispersal) block in Fig. 2.3, the data is RS-encoded. A (204, 188) RS code (shortened from the original $(255,239)$ RS code) is applied to each 188 -byte packet. Fig. 2.6 shows the resulting 204-byte error-protected packet. The RS code produces 16 redundant bytes per 188 bytes used for error correction. This code can correct 8 erroneous bytes per packet, as will be explained in Chapter 4 . 


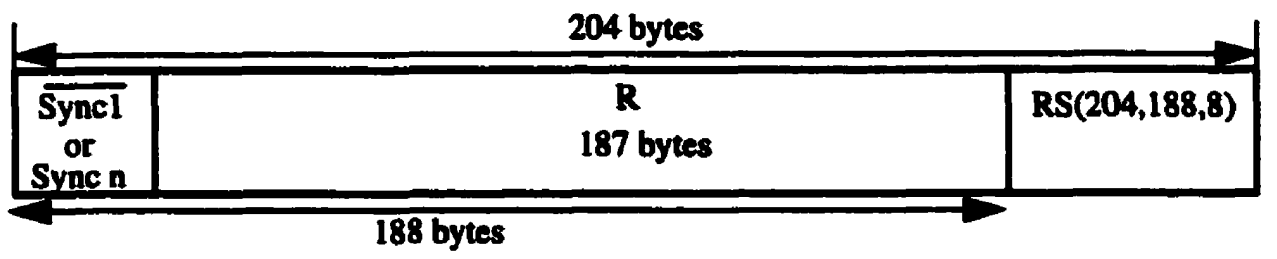

Fig. 2.6: Reed-Solomon RS $(204,188)$ error protection packet

The RS-encoded data is convolutionally byte-interleaved. Fig. 2.7 shows the conceptual scheme of interleaving.

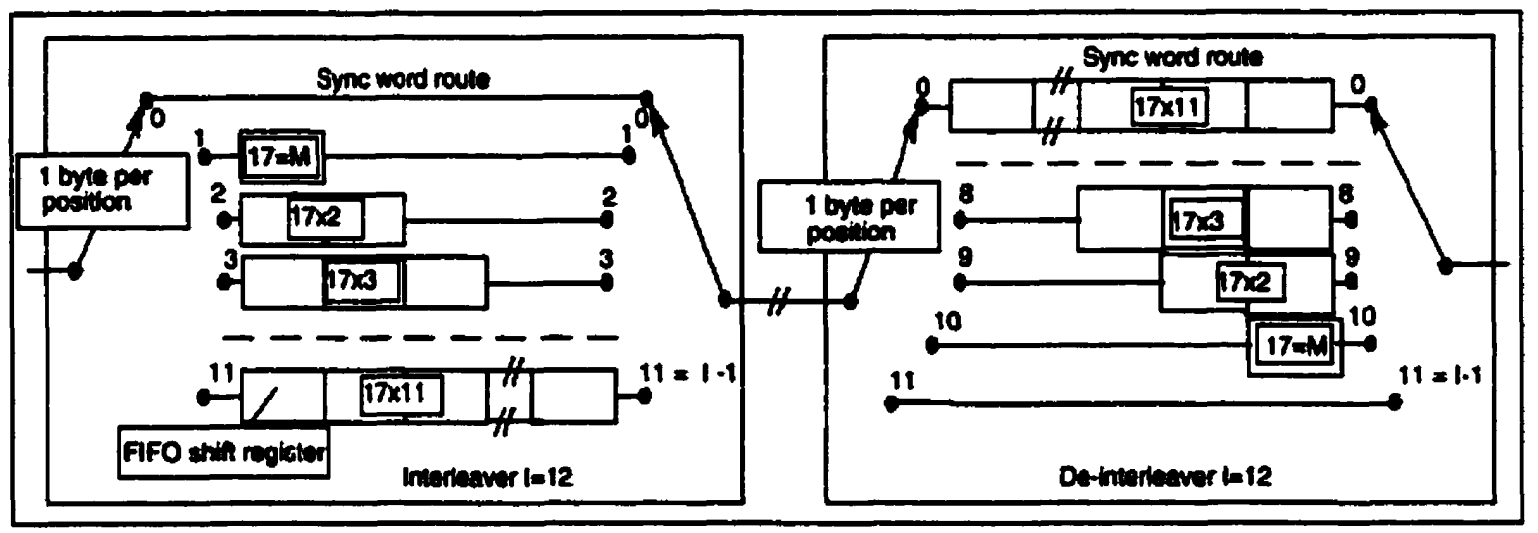

Fig. 2.7: Byte interleaver and de-interleaver defined in DVB standards [1]

The sync-byte is always routed to the branch without any delay elements. Therefore, the syncbytes emerge in their original positions and all other bytes are interleaved. The other branches have multiples of 17 byte-blocks (e.g., path "l" has a 17 byte delay). Each 204-byte RScodeword is split into 17 bytes in each delayed branch, since ( 17 bytes $)^{*}(12$ positions $)=204$ bytes. Fig. 2.8 shows the resulting frame structure, with the non-sync-byte data stream interleaved byte-wise.

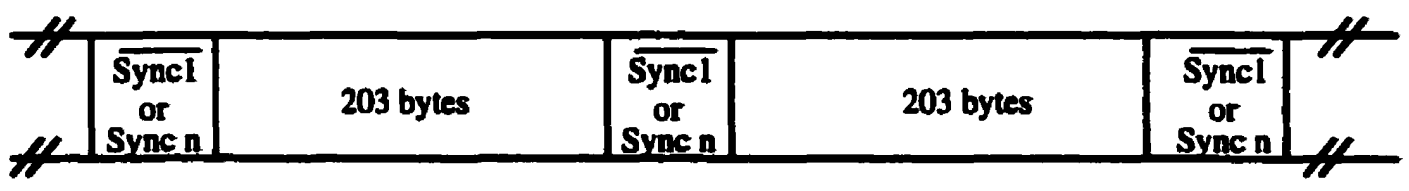

Fig. 2.8: Interleaved frame structure 
The interleaved frames are convolutionally encoded as illustrated in Fig. 2.9. The DVB-S standard allows for a range of punctured code rates based on a 64-state, rate 1/2 convolutional code. The octal representations of the code polynomials are 171 and 133.

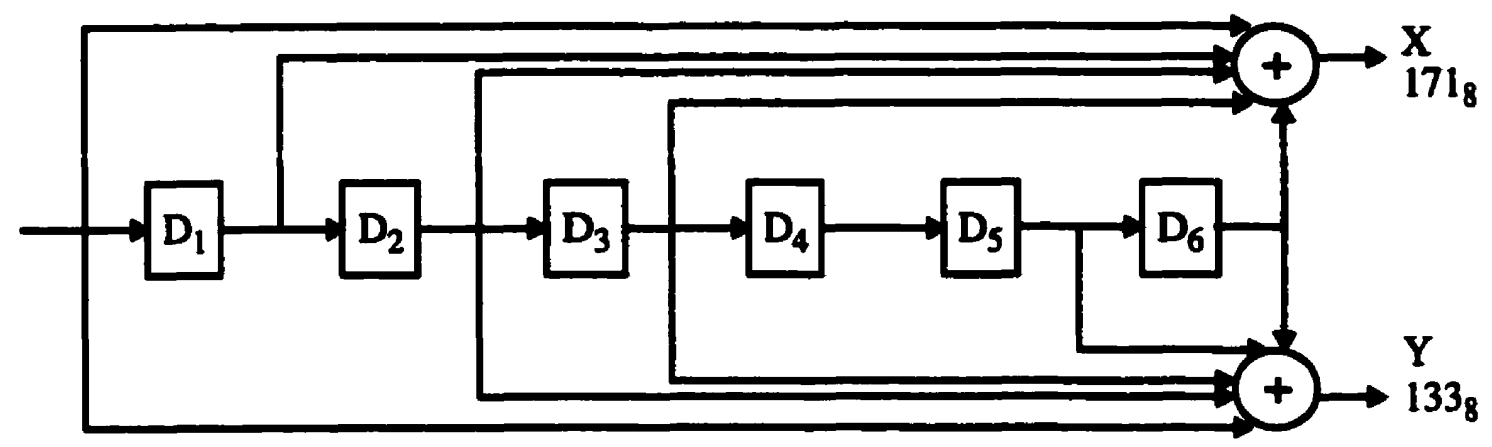

Fig. 2.9: Convolutional encoder

The code rate used can be selected based on the required level of error-protection for a given service or data rate. The convolutional code rate can be any of $1 / 2,2 / 3,3 / 4,5 / 6$ and $7 / 8$. These code rates can be achieved by discarding or "puncturing" certain encoder outputs. The definition of the puncturing pattern to use is given in Table 2.1. The $X$ and $Y$ symbols in the table correspond to the $X$ and $Y$ outputs in Fig. 2.9. A ' $l$ ' in the puncturing pattern indicates that the symbol is transmitted, and a ' 0 ' means the symbol is discarded. The outputs of the puncturing process are the signals $A$ and $B$. The subscripts represent the input symbol number. For example, for the 3/4 code rate, 3 inputs to the convolutional encoder are processed per puncturing period. Without puncturing, this would result in 6 bits output from the encoder. According to Table 2.1, however, the symbols $X_{2}$ (X output for the second symbol) and $Y_{3}$ (Y output for third symbol) are discarded. 
Table 2.1: DVB Punctured code definition [1]

\begin{tabular}{|c|c|c|c|c|c|}
\hline & \multicolumn{5}{|c|}{ Code Rates } \\
\hline & $1 / 2$ & $2 / 3$ & $3 / 4$ & $5 / 6$ & $7 / 8$ \\
\hline \multirow{2}{*}{$\begin{array}{l}\text { puncturing } \\
\text { pattern }\end{array}$} & $X: 1$ & $X: 10$ & $X: 101$ & $X: 10101$ & $X: 1000101$ \\
\hline & $Y: 1$ & Y: 11 & Y: 110 & Y: 11010 & $Y: 11111010$ \\
\hline \multirow{2}{*}{$\begin{array}{l}\text { AB } \\
\text { mapping }\end{array}$} & $A=X_{1}$ & $A=X_{1} Y_{2} Y_{3}$ & $A=X_{1} Y_{2}$ & $A=X_{1} Y_{2} Y_{4}$ & $A=X_{1} Y_{2} Y_{4} Y_{6}$ \\
\hline & $B=Y_{1}$ & $B=Y_{1} X_{3} Y_{4}$ & $B=Y_{1} X_{3}$ & $B=Y_{1} X_{3} X_{5}$ & $B=Y_{1} Y_{3} X_{5} X_{7}$ \\
\hline
\end{tabular}

The $A$ and $B$ signals resulting from convolutional encoding and puncturing are processed by a square-root raised cosine filter (baseband shaping filter with a 0.35 roll-off factor) and a QPSK modulator. The QPSK constellation used in modulation (based on binary A and B values from Table 2.1) is shown in Fig. 2.10.

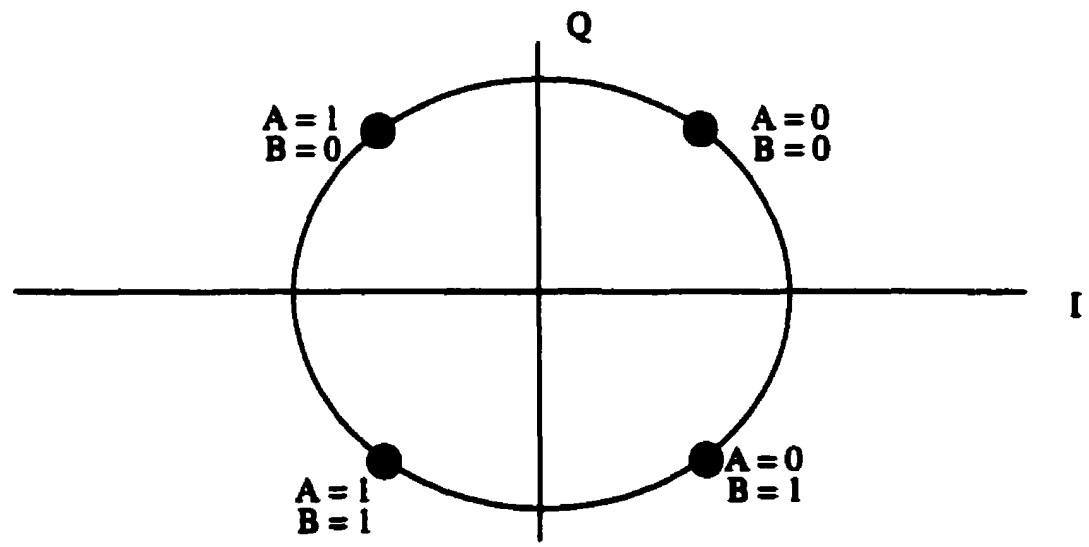

Fig. 2.10: QPSK constellation [1]

A satellite channel is typically modelled as an Additive White Gaussian Noise (AWGN) channel [15]. The error performance requirements for DVB-S are shown in Table 2.2 [1]. The terms "Signal-to-Noise Ratio" (SNR) and $E_{b} / N_{0}$ (where $E_{b}$ represents the energy per bit and $N_{0}$ represents the one-sided noise spectral density) are used interchangeably in this chapter. 
Table 2.2: Error performance requirements [1]

\begin{tabular}{c|c}
\hline Inner code rate & $\begin{array}{c}\text { Required }{ }^{2} \mathrm{E}_{\mathbf{b}} / \mathrm{N}_{\mathrm{o}} \text { for } \mathrm{BER}=2 \times 10^{-4} \text { after } \\
\text { Viterbi decoder }\end{array}$ \\
\hline $1 / 2$ & 4.5 \\
\hline $2 / 3$ & 5.0 \\
\hline $3 / 4$ & 5.5 \\
\hline $5 / 6$ & 6.0 \\
\hline $7 / 8$ & 6.4 \\
\hline
\end{tabular}

a. The figures of $E_{b} / N_{0}$ correspond to the useful bit-rate (before $R S$ coding) and include a modem implementation margin of $0.8 \mathrm{~dB}$ and the noise bandwidth increase from the $R S$ code $(0.36 \mathrm{~dB})$ [1]

These values are used in the Viterbi decoder design of Chapter 3.

A typical DVB-S receiver system, as recommended in the DVB-S standard, is shown in Fig. 2.11. Those components common to other DVB standards are shaded.

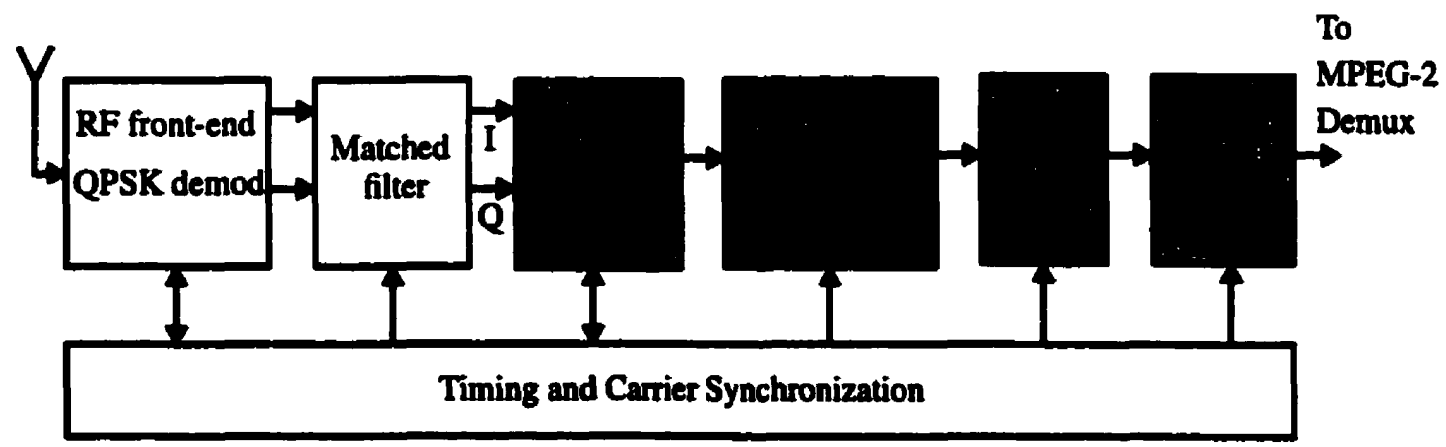

Fig. 2.11: DVB-S receiver block diagram

The RF front end usually consists of a tuner and an IF down converter. The position of the A/D converter is not specified in the DVB-S standard, but if a digital QPSK receiver is used (as in most implementations), the QPSK demodulator would be preceeded by an ADD converter. The matched filter performs the 0.35 raised cosine pulse shaping. The timing and carrier 
synchronization block recovers the demodulator synchronization.

The depuncturing unit must depuncture the input pairs entering the Viterbi decoder according to the perforation pattern given earlier in Table 2.1. The depuncturing unit must try each of the code rates and puncturing configurations until it finds the correct one. This unit can also resolve $\pi / 2$ demodulation phase ambiguity (the condition where the QPSK demodulator is $\pi / 2$ radians out of phase with the modulator). Hardware details of this unit are provided in Chapter 3.

Decoding of the inner, convolutional code is performed by the Viterbi decoder. The DVB-S standard specifies that the BER at the output of the inner decoder must be $2 \times 10^{-4}$ or lower at an $E_{b} / N_{0}$ of $4.5 d B$ (at rate $1 / 2$ ). This BER corresponds to quasi-error free ${ }^{1}(Q E F)$ service after outer code (RS) correction.

A property of the convolutional code used is that, if all input bits to the Viterbi decoder are inverted, the output will be inverted. This can occur in practice if the QPSK demodulator is $\pi$ radians out of phase with respect to the modulator. Fig. 2.10 shows that QPSK symbols are $\pi$ radians out of phase with their logical inverses. This is called $\pi$ ambiguity, and is not detectable by the Viterbi decoder. The sync decoder in Fig. 2.11 monitors the positions of MPEG-2 syncbytes to provide synchronization information for the de-interleaver. It can also resolve $\pi$ ambiguity by ensuring that every eighth sync-byte is inverted. If the opposite condition is detected, the stream can be inverted and passed on to the de-interleaver.

The byte-de-interleaver was presented earlier in Fig. 2.7. Since certain bytes (including syncbytes) were routed to the direct path, these bytes have the largest delay (labelled path " 0 " in Fig. 2.7) in the de-interleaver. The purpose of the de-interleaver is to improve the burst error correction capability of the outer (RS) decoder.

The RS decoder is a byte-based block decoder. The BER at its output is $10^{-10}$ to $10^{-11}(\mathrm{QEF})$ with

1. Quasi-Error Free (QEF) is defined in [1] as being less than one uncorrected error event per hour, correspoading to a BER of $10^{-10}$ to $10^{-11}$ at the input of the MPEG-2 demultiplexer. 
an input BER of $2 \times 10^{-4}$ (provided by the Viterbi decoder).

Next, the randomizing pattern used for energy dispersal is removed and the inverted sync-byte is restored to its normal MPEG-2 sync-byte value. The same circuit, Fig. 2.4, is used for randomizing and de-randomizing.

Between the MPEG-2 transport MUX/demux and the energy dispersal blocks, correct baseband (BB) interfaces must exist to adapt the data structure to the format and protocol required by the MPEG-2 transport components. The DVB standards do not specify the BB interfaces, which would require the MPEG-2 systems standard [34] to design.

The DirecTV Digital Satellite System (DSS), and Primestar DigiCipher-II are other standards for digital video broadcasting via satellite. The standards are proprietary and are not considered in this project. However, based on [19] and [54], these standards have much in common with DVB. The matched filter for DSS and DVB are very similar, with roll-off factors of 0.2 and 0.35 , respectively [17]. The same basic Viterbi decoder could be used, with a modified depuncturing unit (rate $6 / 7$ would need to be added for DSS [17]). A single flexible frame synchronization block and de-interleaver could be used to operate on DVB, DSS or DigiCipher-II type frames. The same basic Reed-Solomon decoder could be used, with a flexble de-shortening unit to insert the correct number of null-symbols in each RS-codeword. More details on the design of a flexible RS-decoder will be provided in Chapter 4.

Some of the components described for DVB-S are identical for other DVB standards. The remaining DVB standards will be explained more succinctly.

\subsubsection{Digital Video Broadcasting - Terrestrial (DVB-T)}

Since DVB-Terrestrial (DVB-T) was introduced after DVB-S, it has not yet been as widely adopted. As of 1999, DVB-T is operating in 17 countries. The DVB-T system is the most complicated DVB system, and the standard is left general enough to apply to many different 
broadcasting scenarios. DVB-T signals are broadcast from fixed transmitting antennas, which are placed on towers to extend the transmitting distance. The RF frequency ranges used are 47 - 68 MHz (VHF I), 174 - $230 \mathrm{MHz}$ (VHF III) and 470 - $862 \mathrm{MHz}$ (UHF IV/V) [16]. The maximum data rate in DVB-T is $40.5 \mathrm{Mbps}$ in a signal bandwidth of $8 \mathrm{MHz}$ [21].

The main difference in signal processing between DVB-S and DVB-T is that the latter uses Orthogonal Frequency Division Multiplexing (OFDM) for modulation. In OFDM, a high overall data rate is achieved by modulating many carriers at low data rates. A major advantage of using OFDM is its robustness in multi-path environments. This gives the use of DVB-T an advantage over DVB-S and DVB-C for coverage of dense, built-up city areas and for mobile reception. Mobile reception of DVB-T signals has been demonstrated at speeds of $300 \mathrm{~km} / \mathrm{h}$ [22].

In DVB-T, MPEG-2 transport MUX packets are created as in DVB-S. Fig. 2.12 shows the DVB$T$ transmitter starting from the MPEG-2 transport MUX output. The signal processing chain from the MPEG-2 transport MUX to the convolutional coding (the shaded blocks in Fig. 2.12) is the same as in DVB-S.

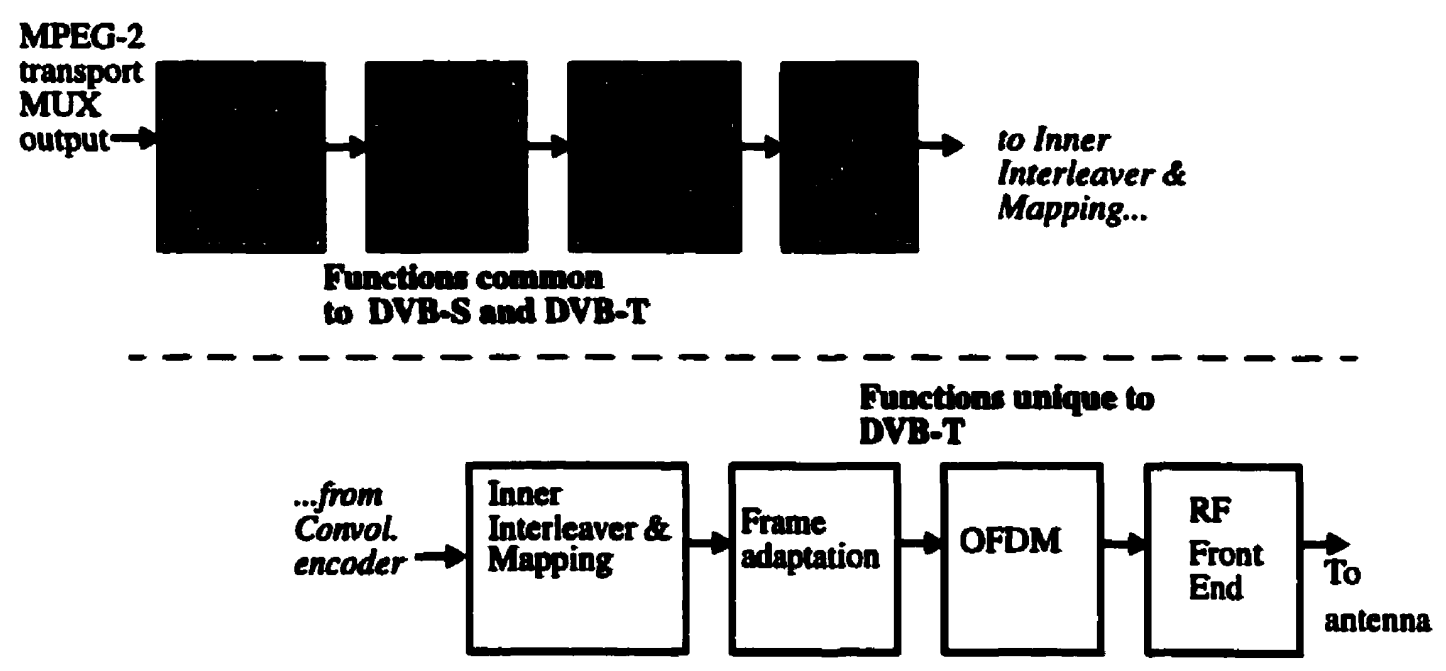

Fig. 2.12: Transmission side of DVB-T [2]

The DVB-T standard allows two-level hierarchical transmission. This means that some data can 
be sent with a high-priority (more robustness to noise) and some with lower priority. The functions of the Viterbi decoder and Reed-Solomon decoder are identical for the two modes. The modulation employed on each carrier can be QPSK, 16 quadrature amplitude modulation (QAM) or 64-QAM, depending on the targeted bit rate. Non-uniform constellations are used in hierarchical transmission modes [2].

A more detailed diagram of the convolutional encoding interface to the inner interleaving is given in Fig. 2.13. DVB-S and DVB-T use the same puncturing pattern, as given in Table 2.1.

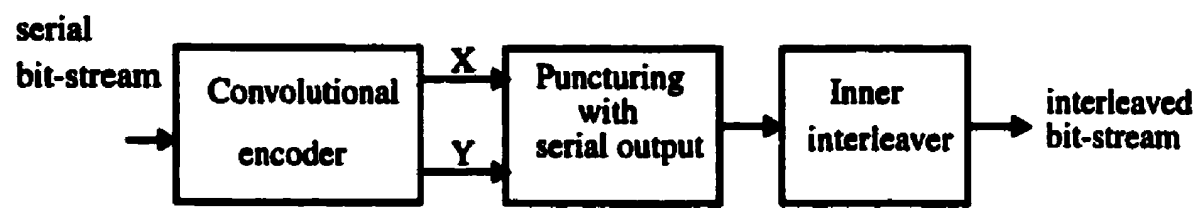

Fig. 2.13: Inner coding/interleaving

In the case of hierarchical transmission, the MPEG-2 MUX output stream is split into a highpriority and a low-priority stream. Each stream is encoded separately, which functionally requires two copies of the shaded blocks of Fig. 2.12 for the transmitter. In this hierarchical mode, the inner interleaver would combine and map the two independent streams [2]. There are different inner interleaving schemes for QPSK, 16-QAM and 64-QAM, depending on whether or not hierarchical modulation is used. Further DVB-T information, including an example of the bitinterleaver, a constellation for non-hierarchical 16-QAM, and the OFDM frame structure is given in Appendix A.

Shortly after the DVB-T standard was specified, several European companies (silicon manufacturers, research centres and receiver manufacturers) started the Digital Video Broadcasting Integrated Receiver Decoder (DVBIRD) project [23], [24]. The aim of the project was to develop a VLSI implementation of a DVB-T receiver. As the DVB-T standard does not suggest a receiver architecture, the basic components of DVBIRD are discussed here as a reference design for a DVB-T receiver. A receiver architecture based on DVBIRD is shown in Fig. 2.14. 


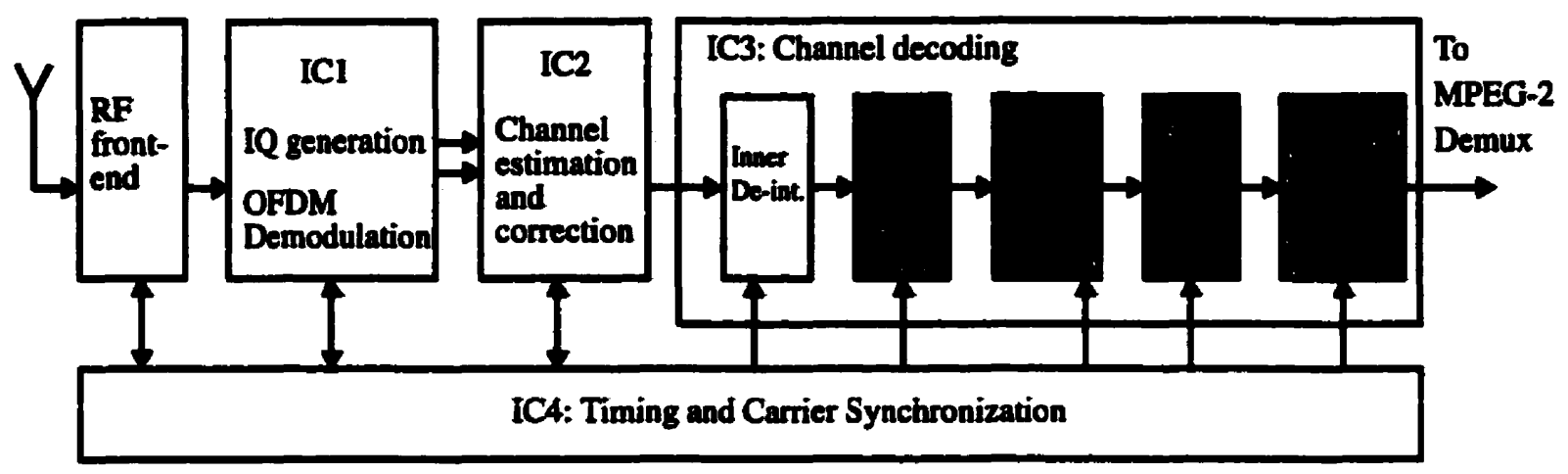

Fig. 2.14: DVBIRD receiver for DVB-T

The DVBIRD receiver consists of an analog front-end and four ICs. The RF front-end consists of a tuner and an IF down-converter. The signal is processed by an Analog-to-Digital Converter (ADC) before being processed by the demodulator IC (IC1). ICl generates the BB signal, corrects the demodulator frequency, and calculates the amplitude and phase of each carrier in the OFDM frame.

IC2 receives the complex BB output of $\mathrm{IC} 1$ and performs channel estimation and correction based on pilots in the received signal. The pilots are interpolated to estimate the channel impulse response. If the receiver is used for portable applications, IC2 interpolates the channel impulse response in time (which requires a large memory to delay demodulated data). The estimated channel response is used to correct the received complex samples. If corrupted carriers are detected, IC2 can signal the Viterbi decoder to ignore the unreliable bits.

IC3 performs inner de-interleaving, Viterbi decoding, outer de-interleaving (as in Fig. 2.7) and Reed-Solomon decoding. Inner de-interleaving functionally involves writing bits into a memory location while reading bits from another address in the same memory. The shaded blocks are the same as in the DVB-S receiver, except that the inputs to the Viterbi decoder can be adjusted based on Channel State Information (CSI) from the channel estimator in DVB-T. IC4 controls the other three chips and performs time and frequency synchronization. It accomplishes this by monitoring the pilots embedded in the transmitted signal (see Appendix A for details.). 


\subsubsection{Digital Video Broadcasting - Cable (DVB-C)}

DVB-C defines the standard for transmission of DVB signals on cable. DVB-C is based on DVB$S$, but the modulation scheme used is Quadrature Amplitude Modulation (QAM) instead of QPSK. The RF frequency ranges used are 47 - $68 \mathrm{MHz}$ (VHF D), 174 - $230 \mathrm{MHz}$ (VHF III) and $470-862 \mathrm{MHz}$ (UHF IV/V) [16]. The maximum data rate in DVB-C is not fixed, but can be as high as 41.34 Mbps in a signal bandwidth of $7.92 \mathrm{MHz}$ [27]. The receiver system is shown in Fig. 2.15, and parts in common with other DVB standards are shaded.

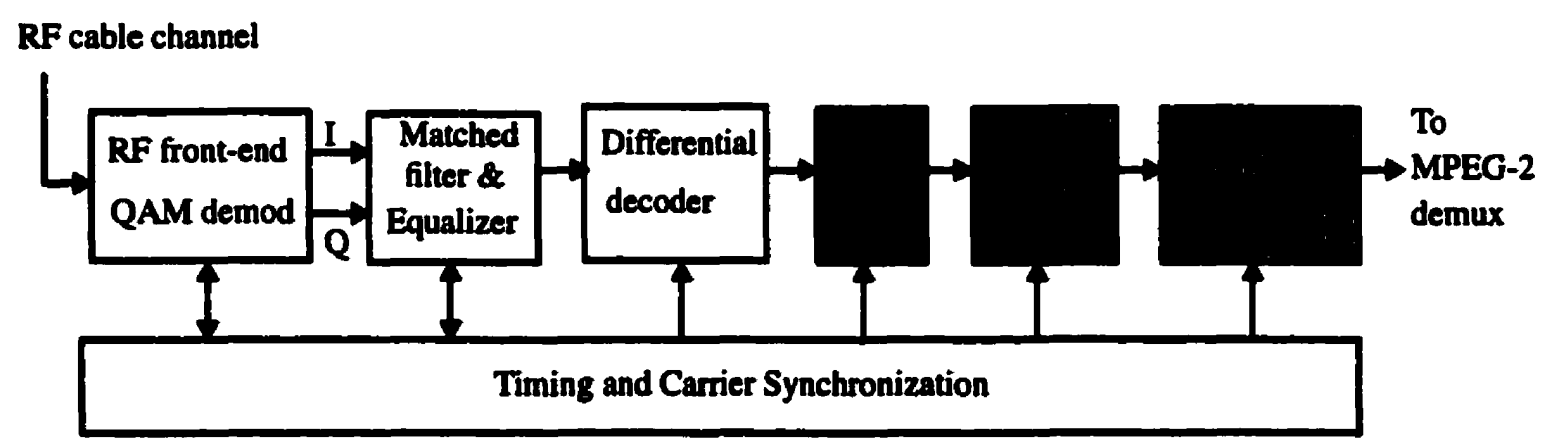

Fig. 2.15: DVB-C receiver

DVB-C uses 16-QAM, 32-QAM or 64-QAM modulation. After demodulation, the signal is applied to a matched filter which uses a 0.15 roll-off matched filter (DVB-S uses a 0.35 roll-off matched filter). The equalizer is used to recover signals which have been distorted by amplitude and phase distortions (from echoes, clipping and non-linearities in the transmission path) [25], [26]. DVB-C uses differential encoding of the QAM symbols at the transmitter, which results in a rotation-invariant constellation. In the receiver, the differential encoding applied at the transmitter is removed, and QAM symbols are mapped to bytes. The byte de-interleaver is the same in all DVB standards, (as shown in Fig. 2.7), and is followed by an RS decoder and descrambler (which are also the same as in the other DVB standards) [3]. 


\subsection{Digital Audio Broadcasting (DAB)}

The DAB standard has been successfully implemented in many countries worldwide, replacing AM/FM transmission with digital MPEG-2 encoded audio. Receivers are commercially available from several manufacturers (eg. Panasonic, Sony). In August 1999, Pioneer made its DAB receivers available in Canada. The Canadian Radio-television and Telecommunications Commission (CRTC) has granted DAB licenses to broadcasters in Toronto, Montreal and Vancouver.

The DAB system is similar to DVB-T in that it mainly transmits its signals terrestrially, using OFDM. The DAB system can operate in four modes for different applications. For example, mode I has a long symbol duration which allows for a long guard interval and hence, a large echo delay. This allows for large transmitter separations in Single Frequency Networks (see Appendix A for details). Other modes are used for local broadcasting, and satellite/complementary terrestrial networks [29]. The structure of DAB transmission frames depends on the mode in use. Mode I DAB frames are organized as shown in Fig. 2.16. As shown, the frame consists of a Synchronization Channel (SC), Fast Information Channel (FIC) and a Main Service Channel (MSC) [4]. The SC consists of a null symbol for frame synchronization, and a reference symbol to provide a reference for differential QPSK (DQPSK). The reference symbol is also used in time and frequency synchronization. The FIC contains Multiplex Configuration Information (MCI) which defines the organization of the sub-channels in terms of their position/size in the MSC and their error protection. The FIC allows the receiver rapid access to information about the transmitted streams. The MSC contains the actual audio/data service components.

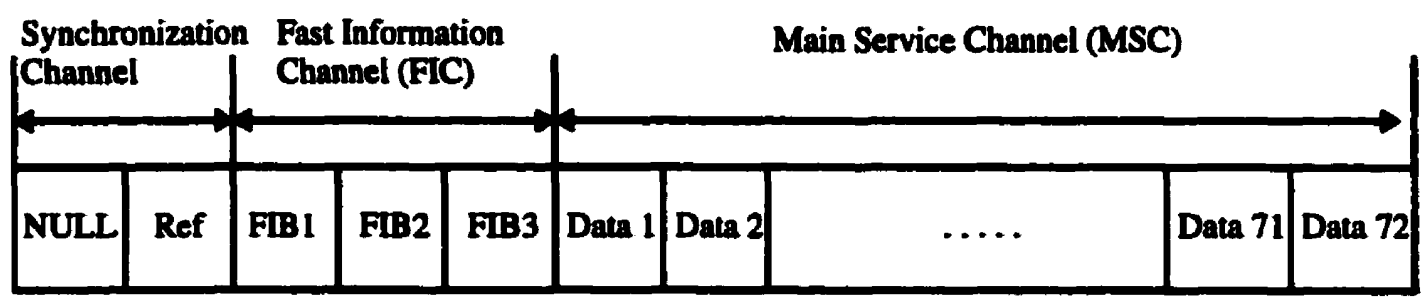

Fig. 2.16: DAB Transmission Frame [29] 
The main features of DAB transmission are the use of OFDM, time and frequency interleaving and convolutional encoding. Only the MSC is time-interleaved to allow the receiver fast FIC acquisition. The RF frequency ranges used are $223-230 \mathrm{MHz}$ (VHF III) and 1452 - $1492 \mathrm{MHz}$ (L-band) [16]. The maximum data rate in DAB is $2.304 \mathrm{Mbps}$ in a signal bandwidth of 1.536 MHz [16].

A typical receiver system is shown in Fig. 2.17. The Viterbi decoder and descrambler have different specifications, but the same basic hardware could be used for the corresponding operations in the DVB standards.

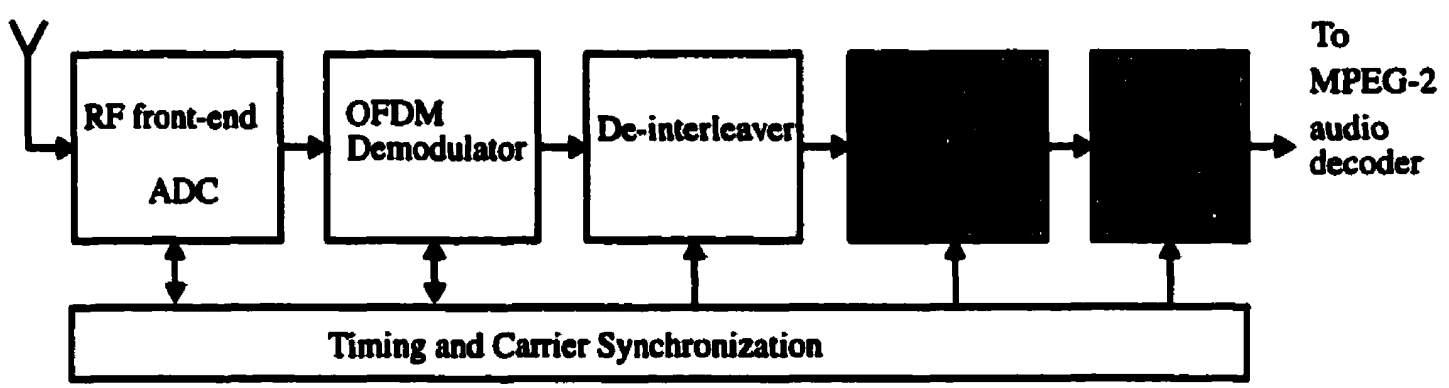

Fig. 2.17: DAB receiver system

The RF front-end consists of a tuner and an IF down-converter. After ADD conversion, the demodulator generates the I and Q signals and passes the reference symbol from the $\mathrm{SC}$ to the synchronization block. An FFT is used in OFDM demodulation. The individual signals are DQPSK demodulated, using the reference symbol to determine the initial carrier phase for each frame. This mitigates the effect of carrier displacement due to the Doppler effect in mobile receivers.

Frequency and time de-interleaving (in that order) are performed next. The interleaving patterns used are given in [4]. The code rate of the Viterbi decoder is controlled by the error protection information specified in the FIC. Twenty-four data rates from 8/32 to 8/9 are supported. Equal Error Protection (EEP) profiles are usually used for data, and Unequal Error Protection (UEP) profiles are typically used for audio. EEP means that all bits within a sub-channel are protected by the same convolutional code rate. UEP is used so that, for example, the less significant bits of 
an audio packet are less protected than the more significant bits (e.g., an error in the leastsignificant bit is less noticeable than an error in the sign bit). The details of UEP/EEP protection profiles are provided in [4]. An embedded core (not shown) could be used to depuncture the data input to the Viterbi decoder based on the FIC data.

The convolutional code used is a rate 1/4 code with 64 states, as in DVB. The code polynomials in octal form are 133, 171, 145 and 133. Energy dispersal is the last step before MPEG-2 audio source decoding, and uses a circuit similar to Fig. 2.4. The DAB PRBS polynomial used for energy dispersal is $1+X^{5}+X^{9}$.

\subsection{Digital Versatile Disc (DVD)}

The DVD writing/reading process is analagous to the transmitting/receiving process described for the other standards. Impairments can include burst noise (from physical damage to the disc), timing jitter, disc tilt, AWGN, intersymbol-interference (ISI) and non-linearities in the readout signal processing [31]. The readout signal data rate of $1 \mathrm{X}$ DVD players is $27.0 \mathrm{Mbps} ; 4 \mathrm{X}$ DVDROM drives run at $108 \mathrm{Mbps}$. Since the DVD readout system is not clearly explained in the literature, since it is based on a closed standard, and because it greatly affects the Viterbi decoder design, it is now explained in detail.

To combat ISI, data to be written to a DVD disc is Run-Length Limit (RLL) encoded. RLL codes are specifed by parameters denoted $(d, k)$. The $d$ parameter specifies the minimum number of zeros between sucessive ones in the binary RLL encoded output. This parameter spreads out transitions, reducing ISI. The $k$ parameter specifies the maximum number of zeros allowed between successive ones. Ensuring adequate transitions aids clock recovery in the receiver and removes low-frequency components from the RLL-encoded signals. Removing low-frequency components is important so that low-frequency components of the information signals do not interfere with servo systems which control the read-head. The specific RLL code used in DVD is a $(2,10)$ code, and is called EFMPlus (Eight-to-Fourteen Modulation Plus). This is an improved version of the EFM (Eight-to-Fourteen) RLL code used for compact discs (CDs), which maps 
eight information bits to fourteen bits. The DVD standard specifies a four-state EFMPlus look-up table which maps eight input bits to sixteen output bits [32].

The RLL encoded data is Non-Retum to Zero Inverted (NRZI) encoded, which decreases the number of transitions in the RLL bit-stream. A high level (' $1^{\prime}$ ) in the RLL-encoded bit-stream is represented by a transition in the NRZI-encoded output. Fig. 2.18 illustrates the process with an example where 8 information bits are RLL-encoded into sixteen bits, which are NRZI encoded. The NRZI waveform is then written to the DVD disc, with pits (low-levels) and lands (highlevels) representing the zeros and ones in the NRZI bit-stream. Note that due to the RLL constraints, the NRZI signal cannot have less than three consecutive identical bits.

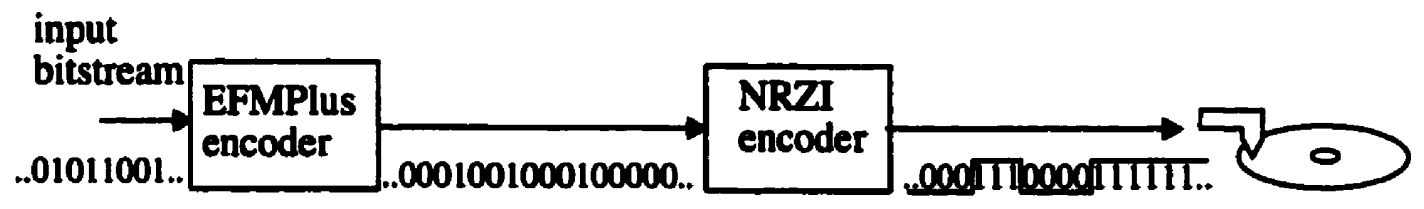

Fig. 2.18: Generation of DVD readout signal

Although it is possible to extract the actual impulse response of the DVD read channel using leastsquares channel identification [31], the read-head of an optical channel can be closely approximated by a Gaussian impulse response:

$$
h(t)=\frac{2 T}{t_{0} \sqrt{\pi}} \exp \left\{-\left(\frac{2 t}{t_{0}}\right)^{2}\right\}
$$

where $T$ is the bit-period and $t_{0}$ is a measure of the Gaussian spot profile width [33]. $T$ is approximately $37.04 \mathrm{~ns}$ and $t_{0}$ was experimentally found to be $150 \mathrm{~ns}$ for a 1 XVD system. Fig. 2.19 shows the normalized impulse response for these parameters. 


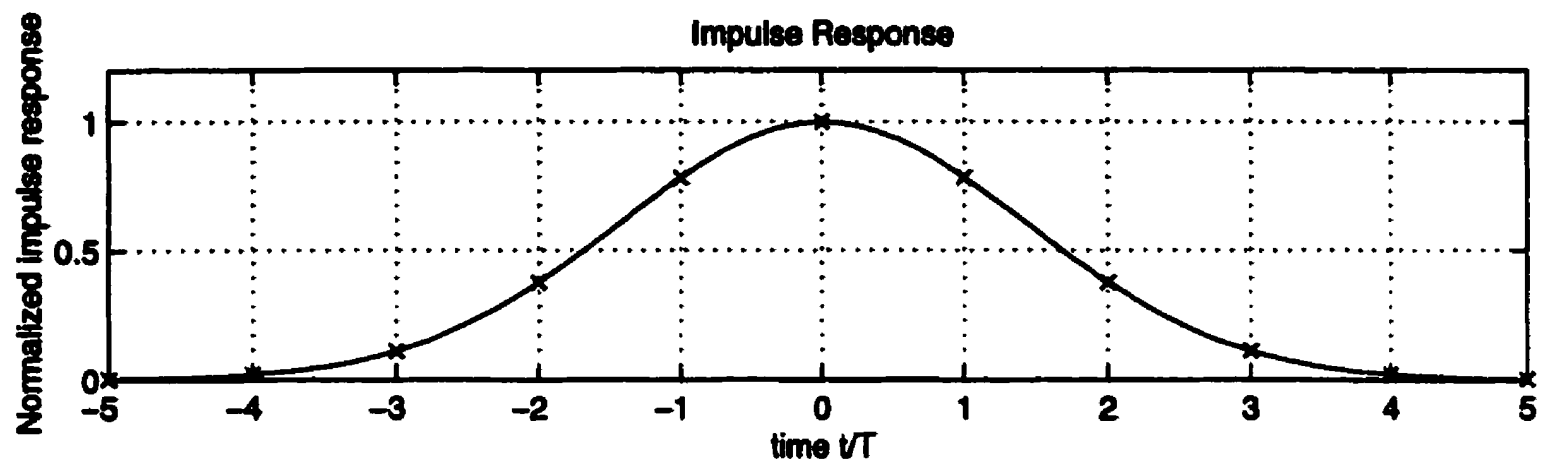

Fig. 2.19: Gaussian impulse response with $t_{0}=150 \mathrm{~ns}, T=37.04 \mathrm{~ns}$

The process of reading from the disc can be mathematically modelled as a convolution of the NRZI waveform with the Gaussian response. This is illustrated in Fig. 2.20, which shows a sample NRZI signal and the corresponding DVD readout waveform.
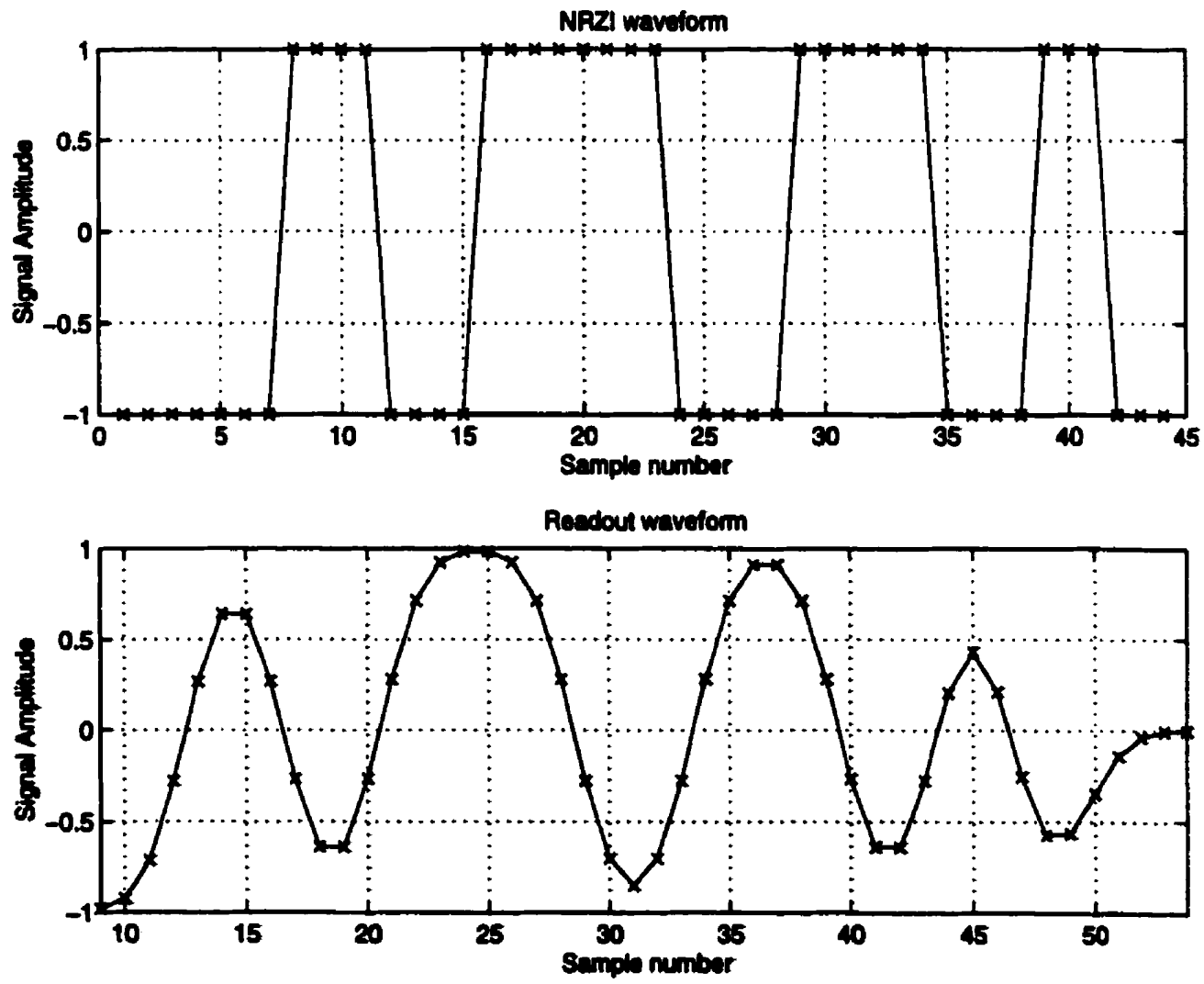

Fig. 2.20: NRZI waveform and corresponding DVD readout waveform 
The readout-signal is, in a sense, a low-pass filtered version of the NRZI waveform and is affected by read-channel impairments (eg. AWGN, nonlinearities). The traditional solution for extraction of the information signal from the DVD readout signal processing is to use a slicer, which processes the readout signal bit-by-bit. Recall that transitions in the NRZI waveform correspond to ones in the RLL signal. Therefore, to recover the RLL signal, the read process consists of detecting the zero-crossings in the readout signal. Ideally, this strategy locates the positions of the ones in the RLL signal. Fig. 2.21 shows an actual readout-waveform and resulting slicer output, with dashed lines showing the correspondence between zero-crossings and decoded bits.

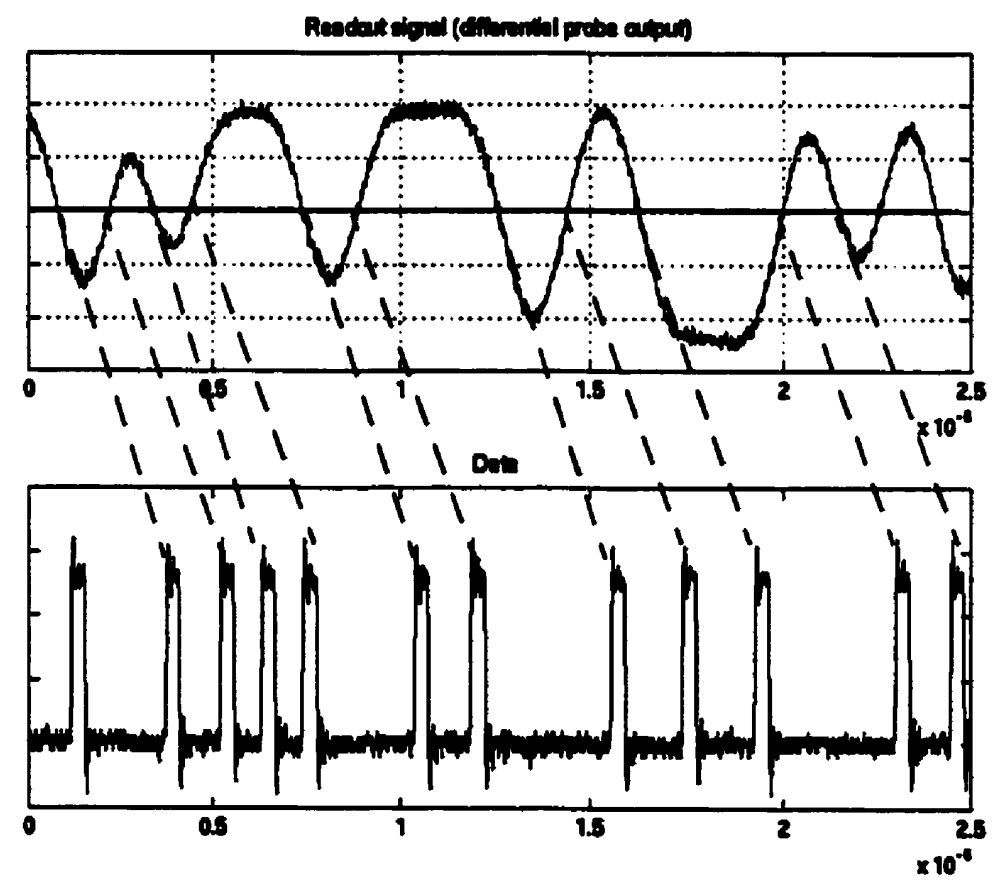

Fig. 2.21: Data extraction using a slicer

First generation DVD players use slicers as illustrated above. Of course, impairments such as ISI degrade the performance of the slicer. To improve performance, a Viterbi detector can be used in place of the slicer. This provides the advantages of MLSD at the cost of added complexity. However, since the VD would be needed for other services in an information furnace, its application to DVD data detection is actually synergistic. Fig. 2.22 shows the read-channel data processing with the Viterbi detector. Although the Viterbi detector and RS-PC decoder have 
different parameters from the Viterbi decoder and Reed-Solomon decoder used in other standards, the same core hardware can be used if designed correctly.

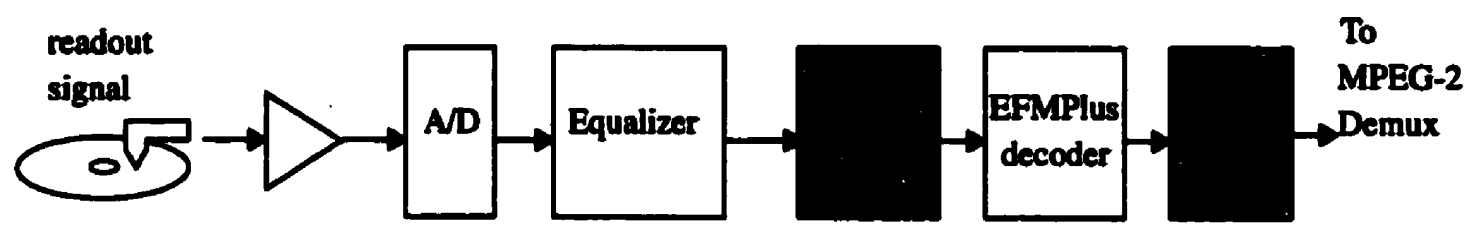

Fig. 2.22: Viterbi-detector based readout system

The DVD standard defines the equalizer which precedes the slicer or Viterbi detector. Since the DVD read channel has a low-pass response, the equalizer is used to boost high-frequencies which were attenuated in the channel. The Viterbi detector produces the maximum likelihood NRZIencoded RLL sequence. The Viterbi detector output is NRZI decoded (each bit XOR'd with the previous bit) to produce the EFMPlus encoded sequence. This is decoded by an EFMPlus decoder to obtain the Reed-Solomon encoded information signal. Chapter 4 will present the Reed-Solomon Product Code (RS-PC) which is specified in the DVD standard to reduce the error rate from $2 \times 10^{-2}$ to $10^{-15}$ [32]. An MPEG-2 demultiplexer splits the signal into its media streams (eg. audio, video), and the streams are source decoded.

\subsection{The Common Elements}

The standards described have many similarities. In particular, the channel decoding operations used have much in common; the receivers must employ a Viterbi decoder and/or a Reed-Solomon decoder. Since all of the described standards involve MPEG-2 source coding, it would be economical to combine the receiver functions so that only one MPEG-2 decoder is required. Although not shown, a microprocessor would be implemented in each of the receiver systems described to provide high level control of the receiver components.

A summary of system parameters is presented in Table 2.3. In cases where parameters are not fixed, practical parameter ranges are given. 
Table 2.3: Summary of system parameters

\begin{tabular}{|c|c|c|c|c|c|}
\hline Parameter & DVB-S & DVB-T & DVB-C & DAB & DVD \\
\hline $\begin{array}{l}\text { RF carrier } \\
\text { frequency }\end{array}$ & $\begin{array}{c}10.70-12.75 \\
\mathrm{GHz}\end{array}$ & \multicolumn{2}{|c|}{$\begin{array}{c}\text { 47 - 68 MHz (VHF I) } \\
174-230 \mathrm{MHz} \text { (VHF II) } \\
\text { 470-862 MHz (UHF IV/V) }\end{array}$} & $\begin{array}{c}\text { 223-230 MHz } \\
\text { (VHF III) } \\
\text { 1452-1492 MHz } \\
\text { (L-band) }\end{array}$ & $n / a$ \\
\hline $\begin{array}{c}\text { Signal } \\
\text { bandwidth }\end{array}$ & $\begin{array}{l}\text { not fixed, } \\
26-54 \mathrm{MHz}\end{array}$ & $6-8 \mathrm{MHz}$ & $7.9 \mathrm{MHz}$ & $1.536 \mathrm{MHz}$ & $-7 \mathbf{M H z}$ \\
\hline Modulation & QPSK & $\begin{array}{l}\text { OFDM using } \\
\text { (4/16/64)-QAM }\end{array}$ & $\begin{array}{c}(16 / 32 / 64) \\
\text { QAM }\end{array}$ & $\begin{array}{l}\text { OFDM using } \\
\text { DQPSK }\end{array}$ & $\mathbf{n} / \mathbf{a}$ \\
\hline $\begin{array}{l}\text { Examples of } \\
\text { noise sources }\end{array}$ & AWGN & $\begin{array}{l}\text { Multi-path, } \\
\text { AWGN }\end{array}$ & Echos, AWGN & $\begin{array}{l}\text { Multi-path, } \\
\text { AWGN }\end{array}$ & $\begin{array}{l}\text { AWGN, burst } \\
\text { noise }\end{array}$ \\
\hline $\begin{array}{l}\text { Maximum } \\
\text { data rates }\end{array}$ & $\begin{array}{l}\text { not fixed, } \\
\text { 40.6 Mbps - } \\
\text { 84.4 Mbps }\end{array}$ & 40.5 Mbps & $\begin{array}{l}\text { not fixed, } \\
41.3 \mathrm{Mbps}\end{array}$ & 2.3 Mbps & $\begin{array}{l}27.0 \text { Mbps at } \\
\text { IX }\end{array}$ \\
\hline $\begin{array}{c}\text { Channel } E_{0} \mathbf{N}_{0} \\
\text { or CNR } \\
\text { (Carrier-to- } \\
\text { Noise Ratio) }\end{array}$ & $E_{b} / N_{0}: 4-7 \mathrm{~dB}$ & CNR:3 - $28 \mathrm{~dB}$ & $\begin{array}{c}\mathrm{E}_{\mathbf{0}} \mathbf{N}_{0}: 14-22 \\
\mathrm{~dB} \\
\text { (estimated } \\
\text { [28] for } \\
\text { 64-QAM) }\end{array}$ & $\begin{array}{l}E_{b} / N_{0}: 4-12 \mathrm{~dB} \\
\text { (estimated [30]) }\end{array}$ & $\begin{array}{c}\mathrm{E}_{\mathrm{b}} / \mathrm{N}_{0}: 8-20 \\
\mathrm{~dB} \\
\text { (estimated } \\
{[31] \text { ) }}\end{array}$ \\
\hline BER spec & $\begin{array}{l}2 \times 10^{-4} @ 4.5 \\
\mathrm{~dB} \text { for rate } 1 / 2\end{array}$ & $\begin{array}{c}2 \times 10^{-4} \bigcirc 3.1 \mathrm{~dB} \\
\text { for QPSK, rate } \\
1 / 2\end{array}$ & $\begin{array}{l}10^{-6} \text { at }>19.5 \\
\mathrm{~dB} \text { (estimated } \\
[28])\end{array}$ & $\begin{array}{c}3 \times 10^{-4} \text { at }>7 \mathrm{~dB} \\
\text { (estimated [30], } \\
\text { mode III) }\end{array}$ & $\begin{array}{l}10^{-3} \text { at }>7 \mathrm{~dB} \\
\text { (estimated } \\
{[31] \text { ) }}\end{array}$ \\
\hline
\end{tabular}

Fig. 2.23 shows the channel decoding parameters for each of the standards. Ideally, each layer would be realized as a single hardware block. However, the objective here is to locate the layers common to as many standards as possible, and focus on those. 


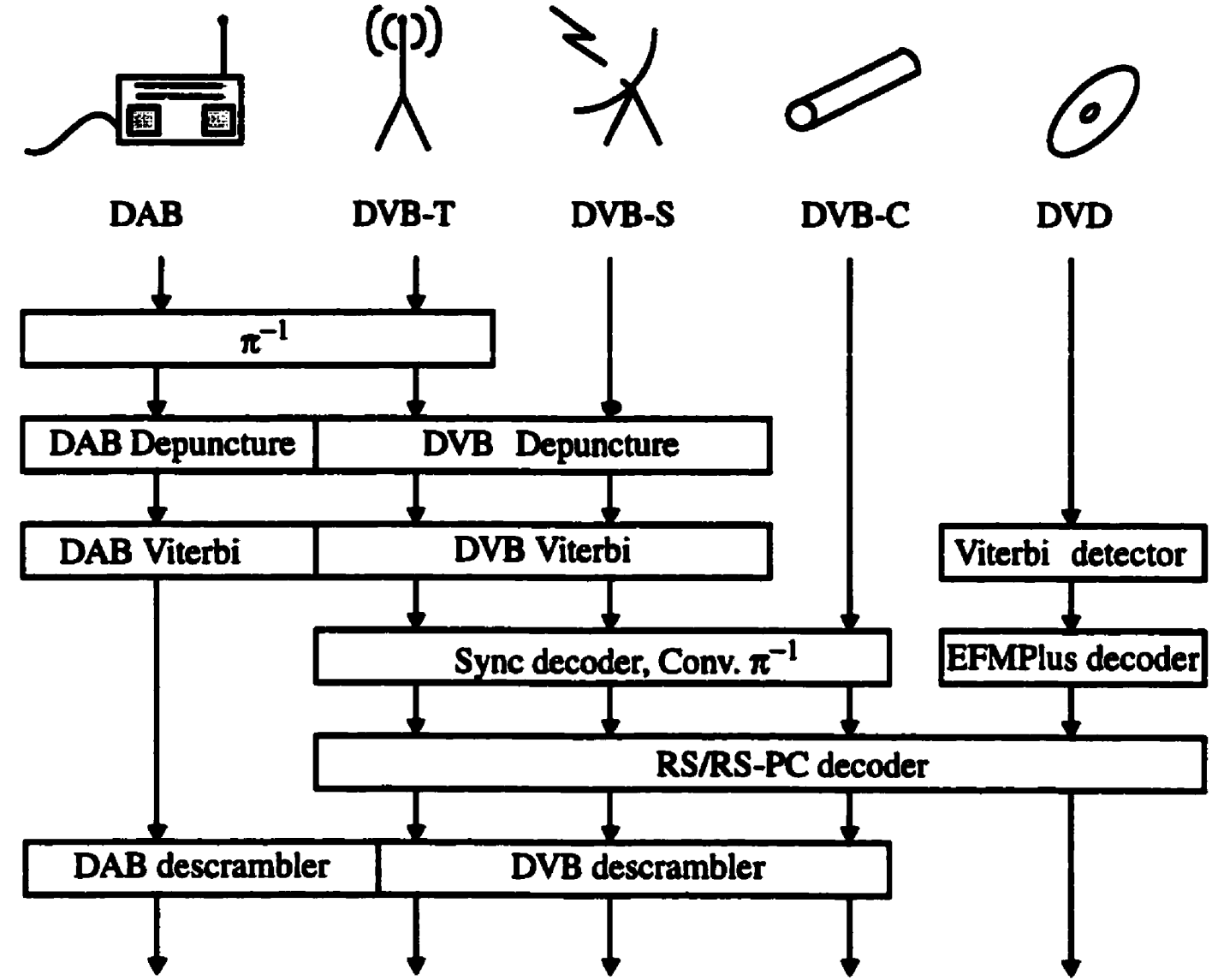

To MPEG-2 demultiplexer and source decoder

Fig. 2.23: Channel decoding common elements ( $\pi^{-1}$ represents a de-interleaver)

Only the terrestrial transmission standards (DAB, DVB-T) employ de-interleaving before the VD. The depuncturing unit is different for DAB, DVB-S and DVB-T. In DAB, the inner code rates used in depuncturing are transmitted in the FIC. Therefore, a controller (e.g., a microprocessor) must monitor the FIC for the inner code rates and signal the depuncturing unit. The depuncturing unit in this case is a collection of $\mathbf{2 4}$ state machines, one for each code rate. Code rates in DVB-T are handled similarly, via the Transmission Parameter Signalling (TPS) carriers in the OFDM signal (see Appendix A for details). A controller monitors the code rate in the TPS signal and forwards it to the depuncturing unit. Unlike DAB and DVB-T, DVB-S does not transmit information on the code rate and must detect it automatically. A depuncturing algorithm from [42] is presented in Chapter 3 which automatically detects the transmitted code rate. 
All three DVB standards have an identical sync decoder, convolutional de-interleaver, and RS decoder. DVB-S and DVB-T have the same basic VD, with a few differences in how the inputs are passed to the VD in DVB-T. In DVB-T, if a higher order QAM than QPSK is used, each symbol carries up to 6 VD inputs. Since the rate 1/2 Viterbi decoder used in DVB-S accepts two inputs at a time, they must be buffered and passed to the VD, two at a time. Another difference in the DVB-T VD is that the VD inputs can be adjusted using channel state information from the transmitted pilots (e.g., values passed on unreliable carriers can be ignored by the Viterbi decoder). DVD uses an RS-PC decoder, which can use the same core Reed-Solomon decoder used for the DVB standards as will be explained in Chapter 4.

No single channel decoding operation is common to all five standards, but examination of Fig. 2.23 reveals the processing layers which are common to most standards. Specifically, Viterbi decoding, Reed-Solomon decoding and descrambling are used in four out of the five standards. Descrambling is performed in the circuit of Fig. 2.4 for DVB, and a very similar circuit is specified for DAB. This operation is simple (i.e., low hardware complexity) and is not worth unifying into a universal operation compatible with all standards. However, the Viterbi decoder and Reed-Solomon decoder designs are complicated and costly enough to consider combining into a reconfigurable ("soft") macro-block. The next two chapters focus on the design of the Viterbi decoder and Reed-Solomon decoder, respectively.

\subsection{Summary}

In this chapter:

- Based on an extensive literature review, this chapter presented a summary of technical details behind the channel decoding elements in important emerging standards for home entertainment set-top box applications. One of these, the DVD readout system, is based on a closed standard; it was therefore explained based on experimental measurements and simulations.

- A graphical illustration of the common channel decoding elements found in the DAB, DVB-S, DVB-T, DVB-C, and DVD standards have been presented in Fig. 2.23. It can be observed that the most advantageous elements to co-integrate are the Viterbi and Reed-Solomon decoders. 


\section{Chapter 3. Viterbi Decoder Design}

Most of the standards considered in Chapter 2 require the use of convolutional codes. The most widely used algorithm for decoding convolutional codes is the Viterbi algorithm (VA), which has a relatively low-complexity implementation. The VA describes a maximum likelihood decoding scheme for convolutional codes. The VA can also be used to recover signals affected by ISI. When used to decode convolutional codes, the device which implements the VA is termed a "Viterbi decoder". When used to mitigate ISI, it is called a "Viterbi detector". When describing components of the hardware design which are common to the decoder and detector architectures, we use the term "Viterbi decoder" (VD).

Chapter 2 showed that a Viterbi decoder is used in DVB-S, DVB-T, DAB and DVD. In this chapter, the VA is presented, and the general structure of a VD is described. The specific hardware architecture of a VD matched to the described standards is explained. Mostly, the requirements of DVB-S and DVD were used in designing the VD.

\subsection{The Viterbi Algorithm}

Recall that the DVB-S, DVB-T and DAB standards use convolutional codes. The Viterbi decoders in the corresponding receivers are based on the Viterbi algorithm (VA). The Viterbi detector which can be used in DVD products for the purpose of combatting inter-symbol interference (ISI) is also based on the VA. Based on the presentation in [37], the VA is introduced as generally as possible, then its applications in convolutional decoding and in mitigating intersymbol interference are explained.

The VA provides maximum a posteriori probability (MAP) estimation of the state sequence of a finite-state discrete-time Markov process in memoryless noise (also called "maximum-likelihood sequence detection" or MLSD). Assuming that time runs from 0 to $K$, the state sequence of the 
Markov process can be represented as a finite vector $s=\left(s_{0}, s_{l}, \ldots, s_{K}\right)$ with each $s_{k}$ representing the state at discrete time $k$. The initial and final states $s_{0}$ and $s_{K}$ are assumed to be known, and each remaining state is an element of a finite set of states $(0,1, \ldots, M)$.

The process is Markov in that the probability $P\left(s_{k+1} \mid s_{0}, s_{1}, \ldots, s_{k}\right)$ of being in state $s_{k+l}$ at time $k+1$ is dependent only on its its state $s_{k}$ at time $k$. That is, $P\left(s_{k+1} \mid s_{0}, s_{1}, \ldots, s_{k}\right)=P\left(s_{k+1} \mid s_{k}\right)$. The transition $t_{k}$ at time $k$ is defined as the pair of states $s_{k+1}$ and $s_{k}$. The process is observed in memoryless noise in that each observation $z_{k}$ depends only on $t_{k}$ at time $k$.

Consider the shift-register model of a Markov process in Fig. 3.1, where each element of the input sequence $\left(u_{0}, u_{l}, \ldots\right)$ can take on any of $m$ values. The function $f\left(u_{k} \ldots, u_{k-v}\right)$ operates on $v$ elements of the input stream at a time to produce a sequence $y$ of values $y_{k}$ which are applied to a memoryless channel. If the function $f$ returns $n$ values at a time, then each $y_{k}$ is an $n$-tuple. The output of the memoryless channel is a sequence $z$ of $n$-tuples $z_{k}$. The state $s_{k}$ of the shift-register process is the sequence $\left(u_{k-1}, \ldots, u_{k-v}\right)$ in the shift-register at time $k$.

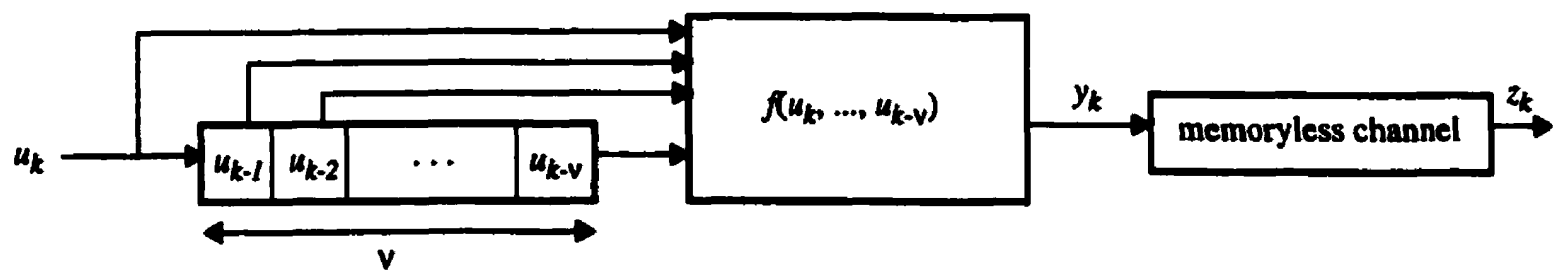

Fig. 3.1: Shift register model of a Markov process observed in memoryless noise

Given a sequence $z$ of observations of a finite-state discrete-time Markov process in memoryless noise, the VA provides the state sequence $s$ with the maximum a posteriori probability $P(s \mid z)$. This MAP rule minimizes the error probability in detecting the sequence s.

A discrete-time, finite-state Markov process can be described by a trellis diagram. Fig. 3.2 shows a trellis representation of a four-state shift-register process. Each node represents a state $s_{k}$ at time $k$, and each edge represents a transition $t_{k}$ to a new state at the next time step. Every possible state 
sequence $s$ of the Markov process traces a unique path through the trellis.

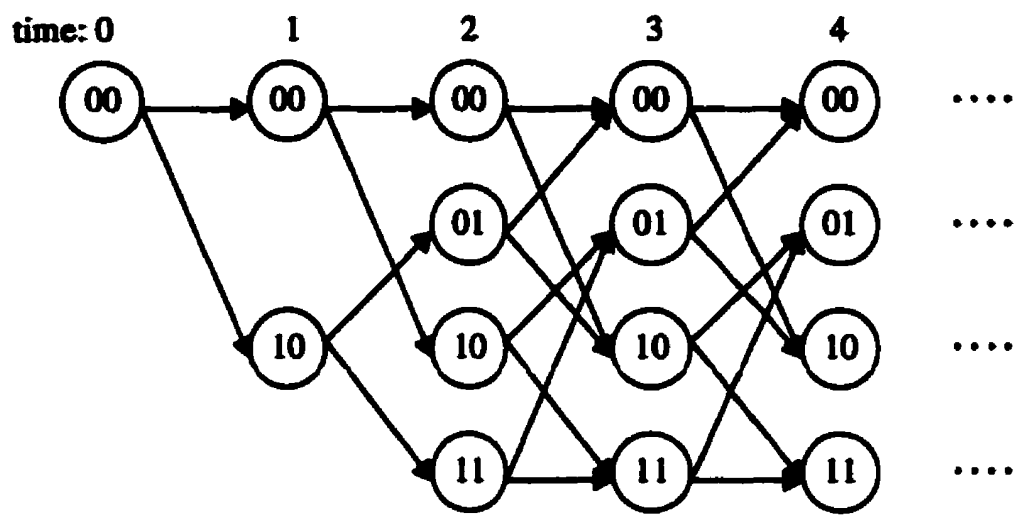

Fig. 3.2: Trellis representation of Markov process

We wish to find the original state sequence $s$ from the sequence of observations $z$. Therefore, $P(s, z)=P(s) \cdot P(z \mid s)$ is to be maximized (this is equivalent to maximizing $P(s \mid z)$ since $P(s, z)=$ $P(s k) \cdot P(z))$. Because the process is Markov and memoryless and because the initial state $s_{0}$ is known,

$P(s, z)=\prod_{k=0}^{K-1} P\left(s_{k+1} \mid s_{k}\right) \cdot \prod_{k=0}^{K-1} P\left(z_{k} \mid t_{k}\right)$, since $P\left(s_{0}\right)=1$

must be maximized. Taking a negative logarithm, this corresponds to minimizing what can be termed the "weight" of the path. The path weight is the summation of its constituent branch (transition) weights:

$-\ln P(s, z)=\sum_{k=0}^{K-1}-\ln P\left(s_{k+1} \mid s_{k}\right)-\ln P\left(z_{k} \mid t_{k}\right)$

Each branch $t_{k}$ has a weight denoted $\lambda\left(t_{k}\right)$, which we define as: 
$\lambda\left(t_{k}\right) \equiv-\ln P\left(s_{k+1} \mid s_{k}\right)-\ln P\left(z_{k} \mid t_{k}\right)$

This branch weight is usually referred to as the "branch metric". The relation to be minimized can be re-written as:

$-\ln P(s, z)=\sum_{k=0}^{K-1} \lambda\left(t_{k}\right)$

An initial segment of the trellis $s_{0}^{k}=\left(s_{0}, s_{l}, \ldots, s_{k}\right)$ represents the path segment starting at node $s_{0}$ and ending at $s_{k}$. The segment of lowest weight is called the survivor of node $s_{k}$. Each node at time $\boldsymbol{k}$ has one survivor sequence. A key idea in the VA is that the shortest complete path through the trellis must start with one of these survivors. At each time step of the VA, only one survivor per state needs to be stored, along with their corresponding weights.

The VA can be described as follows:

\section{Storage:}

$k \quad$ time index

$\hat{s}\left(s_{k}\right), 0 \leq s_{k} \leq M \quad$ survivor ending in $s_{k}$

$\mathrm{L}\left(s_{k}\right), 0 \leq s_{k} \leq M \quad$ survivor length (also called path weight, path metric, state metric)

Initialization:

$k=0$

$s\left(s_{0}\right)=s_{0}$

$L\left(s_{0}\right)=0$

\section{Becursion:}

$\mathrm{L}\left(s_{k+1}, s_{k}\right)=\mathrm{L}\left(s_{k}\right)+\lambda\left(t_{k}\right)$ for all transitions $\left(t_{k}=s_{k+1}, s_{k}\right)$

Find $L\left(s_{k+1}\right)=\min _{s_{k}} L\left(s_{k+1}, s_{k}\right)$ 
for each $s_{k+1}$. Store $L\left(s_{k+1}\right)$ and the corresponding survivor $\hat{s}\left(s_{k+1}\right)$.

Set $k=k+1$, and repeat until $k=K$.

The VA completes with the lowest weight-path stored as the survivor $\hat{s}\left(s_{K}\right)$.

In practical situations, the a measure of the survivor length (also called "merge depth") $\delta$ must be truncated to keep storage requirements reasonable. This does not adversely affect performance if $\delta$ is high enough, since survivors usually merge and agree on a common ancestral path through the trellis. As an example, survivors usually merge within $5 v$ time-steps for rate $1 / 2$ convolutional codes in moderate signal-to-noise ratios [38]. Another practical detail is that as path metrics grow in size, a mechanism must exist to handle overflow. Further details and an example of the VA are given in [37].

The DVB-S, DVB-T and DAB standards employ a convolutional code for error-correction. The DVD readout-signal is affected by intersymbol-interference. Because the operations of convolutional encoding and reading from a DVD disc can be modelled as a finite-state Markov process, the Viterbi algorithm can be applied to both problems.

The shift-register model of Fig. 3.1 can be used to model convolutional encoding. An encoder for a rate $k / n$ convolutional code encodes an input $k$-tuple into an output $n$-tuple. The convolutional code rate can be thought of as the ratio of information-symbols to total symbols to be transmitted. To simplify the following discussion, we consider the case where $k=1$ (rate $1 / n$ codes). A vmemory convolutional encoder consists of a v-stage shift-register with the outputs of certain stages added modulo-2 to produce the encoded symbols.

Fig. 3.3 shows the rate $1 / 2$ convolutional encoder diagram for DVB which corresponds to the shift-register model of Fig. 3.1. Each $\boldsymbol{u}_{k}$ is a single information bit. Certain bits of the shiftregister are input to two modulo-2 adders to produce two outputs, $X$ and $Y$. These outputs are subsequently parallel-to-serial converted to form a stream which corresponds to the sequence of $y_{k}$ values in Fig. 3.1. The artangement and number of modulo-2 adder inputs can be varied to 
change the type of code. The shift-register taps for the X-output's modulo-2 adder in Fig. 3.3 can be represented in a binary vector (1111001), where a ' 1 ' indicates a connection from an input bit to the adder. This vector is usually written in octal form as 171 . Similarly, the taps for the $Y$ output can be expressed as the vector (1011011), or 133 in octal form.

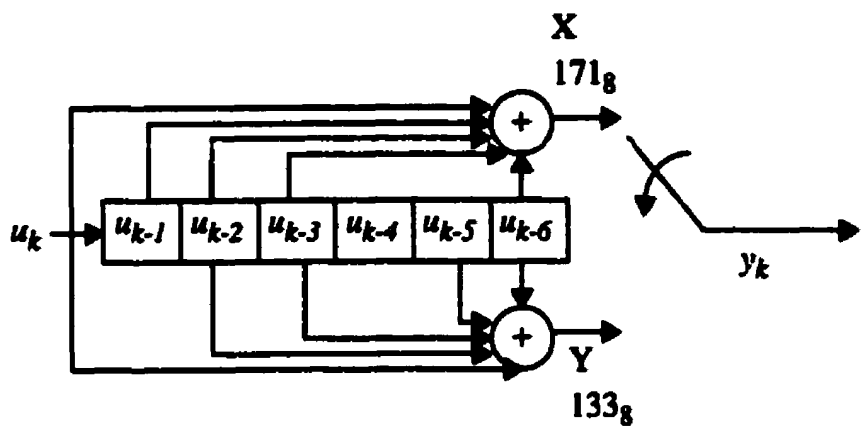

Fig. 3.3: Convolutional encoder for DVB

The application of the encoder's output sequence to a channel with memoryless noise completes the correspondence with the general model of Fig. 3.1.

The intersymbol interference (ISI) inherent in DVD readout signals can also be modelled by a shift-register process. ISI occurs in the DVD readout signal because the impulse response (section 2.3, equation (1)) of the optical pick-up used in the DVD read head spans several bitintervals. Fig. 3.4 illustrates the ISI shift-register process, which models the convolution of the NRZI waveform with the channel impulse response. Samples $u_{k}$ of the NRZI waveform are input to the shift register, and are multiplied by samples $h_{j}$ of the impulse response. These products are summed to form samples $y_{k}$ of the readout signal.

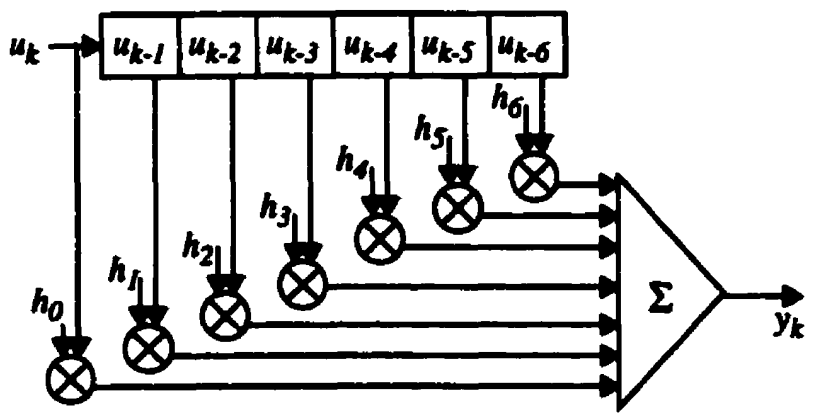

Fig. 3.4: DVD ISI shift-register process 
The samples $y_{k}$ are sent through a channel with memoryless noise, as in the general model of Fig. 3.1.

\subsection{Viterbi Decoder Structure}

The Viterbi Decoder (VD) implements the Viterbi algorithm, and typically consists of three main components: the Branch Metric Generator (BMG), the Add-Compare-Select (ACS) unit, and the Survivor Memory Unit (SMU). An Output Decision Device (ODD) is used to select the output bit from all of the survivor paths. There are several design parameters of the VD components which must be selected through performance analysis. For the BMG, the input quantization and type of branch metric calculation must be selected. The bit-widths used for path metric storage in the ACS unit must be determined and the decoding depth $\delta$ must be selected for the SMU/ODD combination. This section presents the VD simulation results and parameter selection for the core VD components shown in Fig. 3.5. The Depuncturing Unit (DU) which is used to format the VD inputs according to the code rate is described at the end of the chapter.

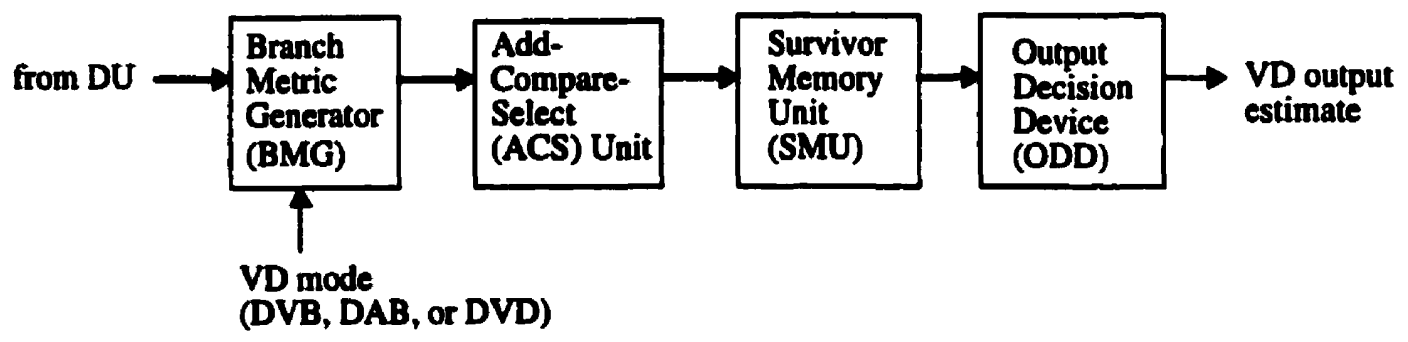

Fig. 3.5: Viterbi Decoder structure

\subsubsection{Branch Metric Generator}

With respect to a VD which operates with only a single standard, most of the flexibility in a multistandard VD is in the Branch Metric Generator (BMG). This section gives examples of branch metric calculation, presents simulation based constraints on the BMG design, and outlines the high level structure of the BMG. 
Recall that the recursion step in the VA requires the calculation of branch metrics $\lambda\left(t_{k}\right)$ for all transitions $t_{k}$. These branch metrics are calculated based on the received symbols, as explained in the following example. Fig. 3.6 shows a convolutional encoder for a binary rate $1 / 2, v=2$ code, and one stage of the corresponding trellis. There are two branches leaving each state $s_{k}$ : the upper and lower branches correspond to a ' 0 ' and ' 1 ' input $u_{k}$, respectively. Above each transition is its expected $y_{k}$ encoder outputs (note that for this rate $1 / 2$ code, $y_{k}$ is the 2-tuple XY). Note that different transitions $t_{k}$ can have the same "expected" channel output $y_{k}$.
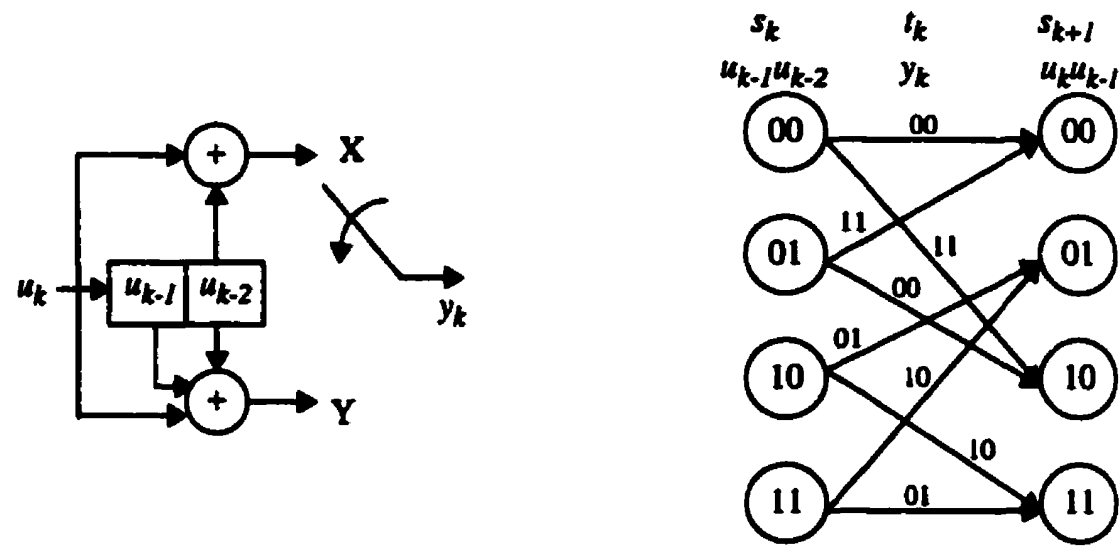

Fig. 3.6: Convolutional encoder and corresponding trellis

The BMG of the VD computes the branch metrics $\lambda\left(t_{k}\right)$ for all transitions. For AWGN, the branch metric for a given transition can be computed as the squared difference between the channel output and the expected output for that transition": $\left(z_{k}-y_{k}\right)^{2}$. This metric is called "squared Euclidean distance" (also referred to as the "L1 norm"). Hardware complexity can be reduced ${ }^{2}$ by using the sub-optimum "Euclidean distance" (also referred to as the "L2 norm") metric: $\mid z_{k}$ $y_{k} l$. A simpler distance measure which results in lower performance is "Hamming distance". This involves quantizing channel outputs to hard decisions and calculating each branch metric by

1. Since the term $P\left(s_{k+1} \mid s_{k}\right)$ in equation (2) is typically a constant for all valid transitions in the shift-register process [37], it can be ignored when comparing different $\lambda\left(t_{k}\right)$ values for a given $z_{k}$. In AWGN, the term $-\ln P\left(z_{k} \mid t_{k}\right)$ in equation (2) is proportional to $\left(z_{k}-y_{k}\right)^{2}[37]$.

2. There is actually a simplification which results in a lower complexity squared Euclidean distance computation: $\left(z_{k}-y_{k}\right)^{2}=z_{k}^{2}-2 z_{k} y_{k}+y_{k}^{2}$. If the expected value is +1 or -1 , then the squared terms can be ignored and adding/subtracting $z_{k}$ would be equivalent to the squared Euclidean distance computation. 
computing the sum of XOR comparisons with the expected values.

The $y_{k}$ samples are modulated, sent through a noisy memoryless channel, and are demodulated at the receiver. At some point, the received signal is AD converted. As an example, assume that the resulting $z_{k}$ symbols are quantized to 4-bits, with 0000 corresponding to ' 0 ' and 1111 corresponding to ' 1 '. If the received $z_{k}$ is the 2-tuple $(0010,1110)$, the branch metric for the transition from state 10 to state 01 with expected output $(0000,1111)$ would be $(0010-0000)^{2}+$ $(1110-1111)^{2}=0101$. In general, for a binary rate $1 / n$ code, there are $2^{n}$ possible expected channel outputs, which corresponds to $2^{n}$ possible branch metrics. In this case, the BMG must calculate $2^{2}=4$ branch metrics and forward them to the ACS unit which uses the branch metrics in its path metric calculation.

Fig. 3.7 shows an example of a $v=2$ ISI shift-register model, impulse response, and trellis stage. In this example, the input stream $u$ is a DVD NRZI stream (+1's and -1 's), and the ISI coefficients are $h_{l}=0.9$ and $h_{0}=h_{2}=0.5$. As an example of a squared Euclidean distance calculation, if a $z_{k}$ of 1.8 is received, the branch metric for the transition from state " $-1-1$ " to state " $-1-1$ " would be $(1.8-(-1.9))^{2}=13.69$.
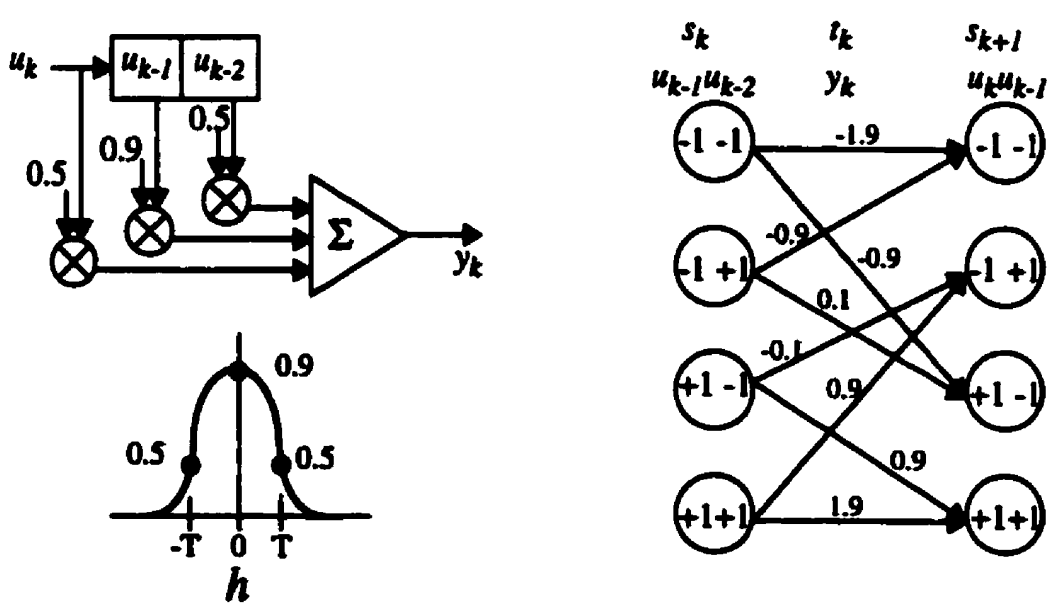

Fig. 3.7: ISI shift-register process and corresponding trellis

To accomodate different standards based on a $v=6$ shift-register process, the most signficant changes in VD structure are in the BMG. Through BER simulations, we determine a suitable 
branch metric type and the required level of input quantization.

As explained in Chapter 1, DVB-S and DVD are the more important standards considered. The VD operation according to these standards was therefore simulated. DVB-S simulations were performed using an AWGN channel. DVD simulations modelling AWGN and ISI were also performed, but more accurate DVD simulations from [31] are considered here, which also model non-linearities and timing jitter.

Fig. 3.8 shows the BER performance of DVB-S at rate $1 / 2$ coding using three different types of branch metrics. The rate $1 / 2$ BER specification given in the DVB-S standard is $2 \times 10^{-4}$ at an SNR of 4.5 dB. Only Euclidean distance squared and Euclidean distance meet the requirement, with Euclidean distance exceeding the SNR requirement by approximately $0.95 \mathrm{~dB}$. Since the degradation in using non-squared Euclidean distance is relatively low (0.37 $\mathrm{dB}$ at a BER of $2 \mathrm{x}$ $\left.10^{-4}\right)$, and since its hardware complexity is significantly lower, it is selected for use in the hardware implementation. It is assumed that the Euclidean distance is a sufficient metric for DAB and DVB-T because of the low gain between Euclidean distance and Euclidean distance squared. DVD simulations show a degradation of less than $0.5 \mathrm{~dB}$ in using Euclidean distance, which is also quite acceptable since the VD gives about $4.5 \mathrm{~dB}$ coding gain over the standard slicer [31]. 


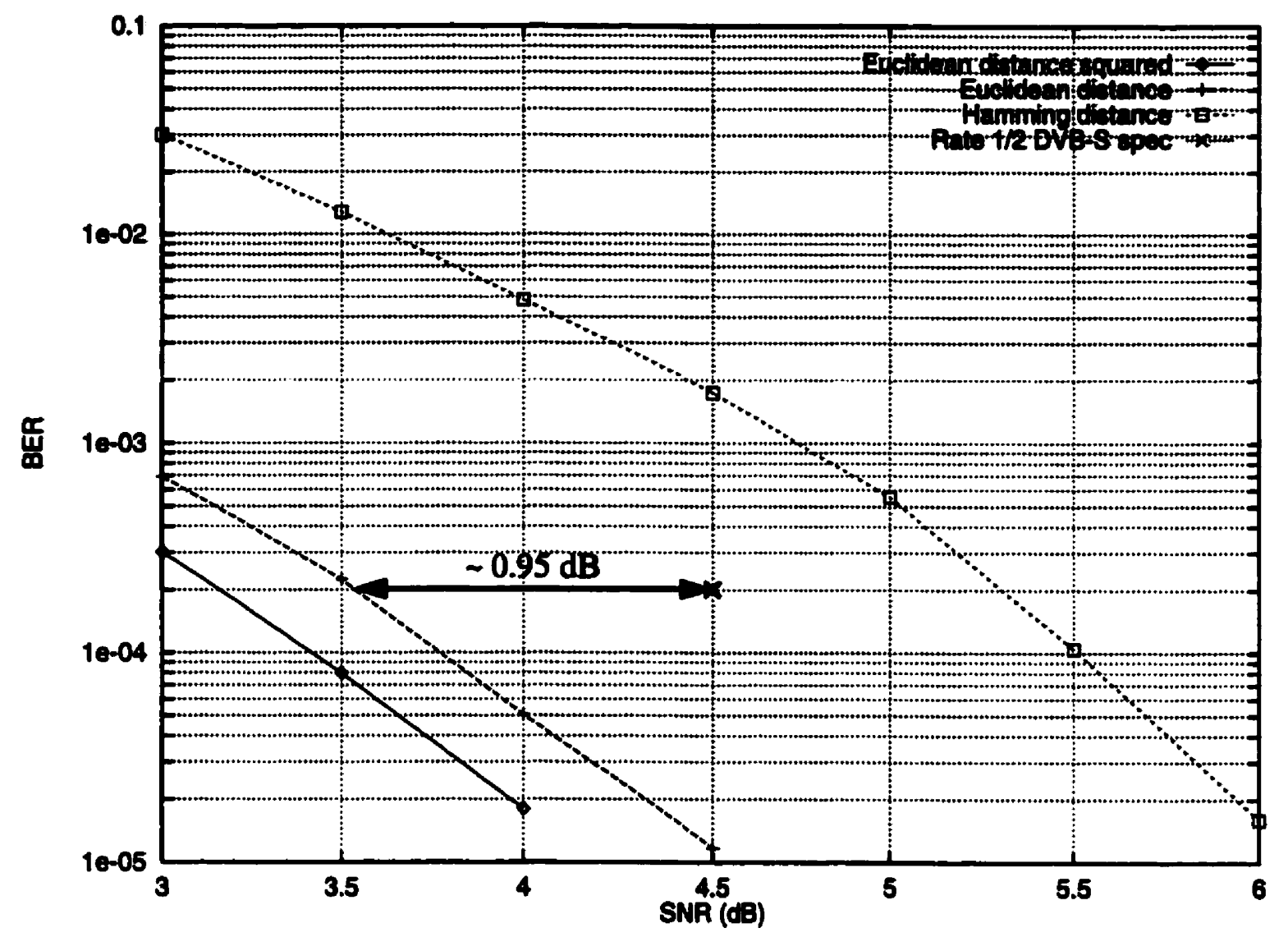

Fig. 3.8: BER performance for different branch metrics

Different levels of quantization for the VD inputs using Euclidean distance is shown in Fig. 3.9. At $2 \times 10^{-4}$, there is no significant difference between 4 and 5 bits of quantization which meet the DVB specification by about $1.15 \mathrm{~dB}$. Since DAB requires 4-bit quantization [30] and the DVBIRD receiver for DVB-T uses 4-bit quantization [23], 4 bits of quantization is used for the standards requiring convolutional decoding. In DVD, 5-bits of quantization should be used [31]. Since DAB requires 4 input symbols, each 4-bits, there are a total of 16 input bits to the BMG. In DVD, a single input symbol is processed at a time. Therefore, using 5-bits in DVD mode is possible by sharing the pins of two DAB symbol inputs, as will be shown in Chapter 5. 


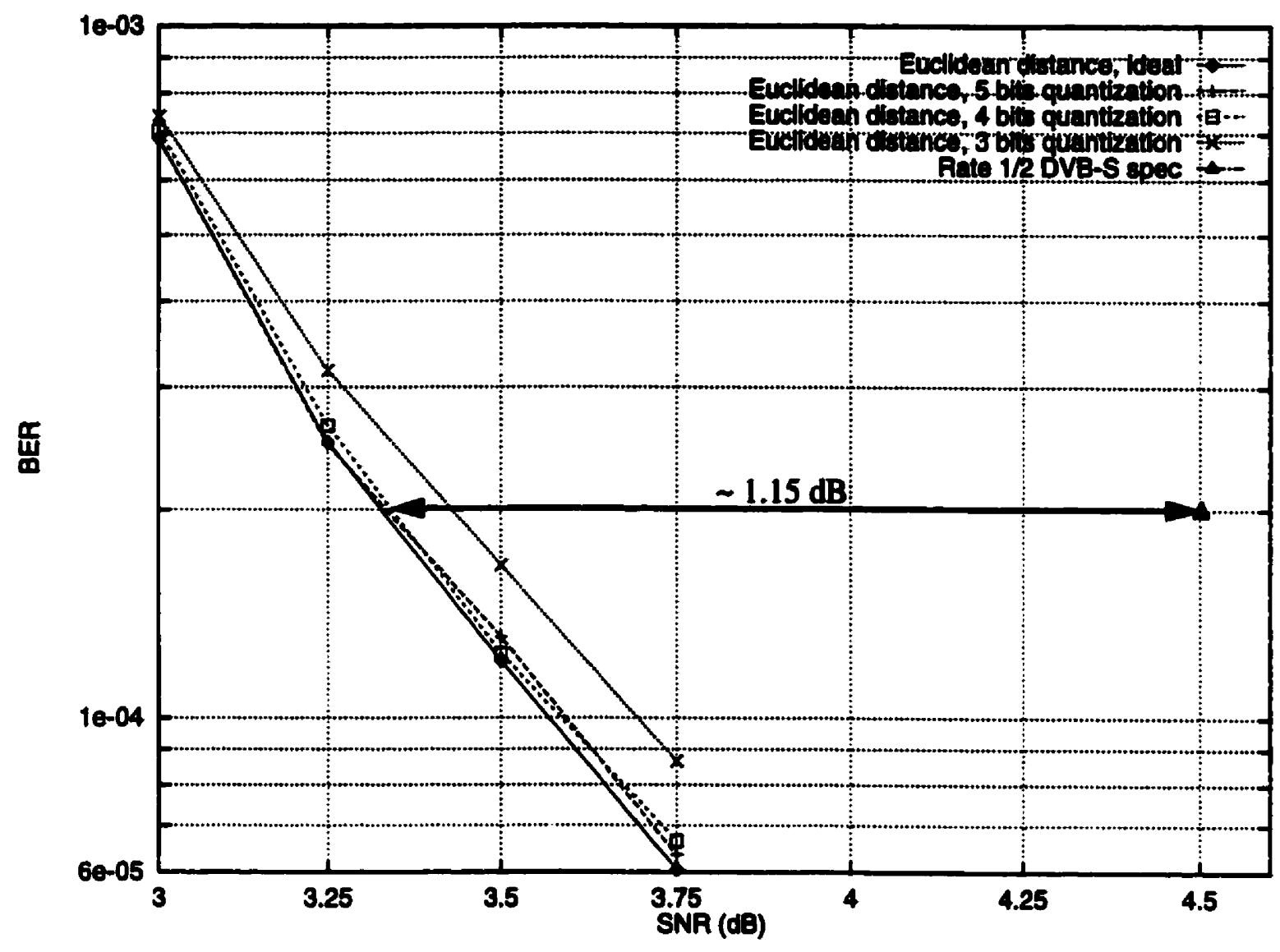

Fig. 3.9: BER performance for different quantization levels

A QPSK constellation quantized to 4 bits is shown in Fig. 3.10, with A and B representing the bit pairs input to the modulator. 


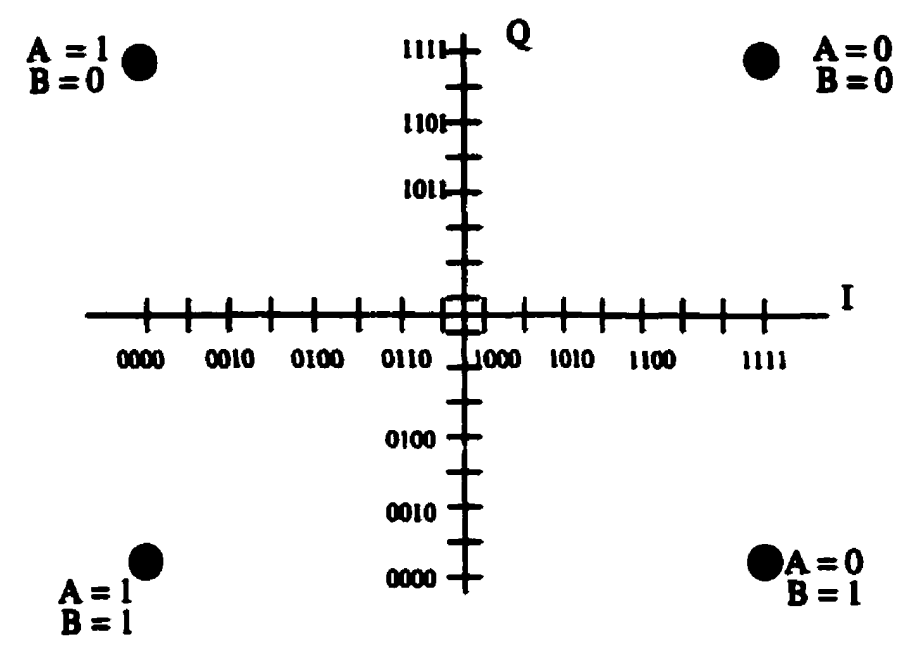

Fig. 3.10: Quantized Constellation

The sequence of values on each axis is $0000,0001,0010, \ldots, 1111$. This allows for a simplification in calculating the Euclidean distance. The quantized inputs will only need to be compared to the expected values 0000 and 1111 . Consider a DVB input symbol represented as $\left(z_{l}, z_{Q}\right)$. The result $b_{l}$ of $\left|z_{l}-0000\right|$ is simply $z_{l}$. The result $b_{l}$ of $11111-z_{l}$ would be $z_{l}$ bitwise XOR'd with 1111. The same applies for the component $b_{Q}$ correponding to $z_{Q}$, and the branch metric $b_{I Q}$ would then be $b_{l}+b_{Q}$. Each branch metric can therefore be calculated with a maximum of 8 XOR gates and one 4-bit adder for DVB-S, which results in a maximum 5-bit branch metric. In DAB, there are 4 input symbols used per branch metric. The branch metrics are calculated in the same way as in DVB-S for the four components $b_{l}, b_{Q}, b_{J}$ and $b_{R}$. This means that the maximum Euclidean distance branch metric is $(1111)+(1111)+(1111)+(1111)=$ 111100. Since DVD branch metric calculation involves a subtraction of two 5-bit numbers, DVD branch metrics are 5-bits. Therefore, the flexible branch metric generator uses 6-bits for each branch metric to accomodate the DAB standard.

Fig. 3.11 shows a high-level diagram of the flexible branch metric generator. Since DAB and DVB calculate their branch metrics in a similar way, one sub-block can be used to calculate both DAB and DVB branch metrics. There are three decoding modes (DAB, DVB and DVD), thus two bits (labelled "decoding mode" in Fig. 3.11) are used to indicate the type of branch metric computation to perform. In order to perform a branch metric calculation, DVD processes one 
channel output, DVB processes two channel outputs and DAB processes four channel outputs. Since a maximum of four channel outputs could be required for an individual branch metric computation, there are four inputs $I, Q, J$ and $R$ to the branch metric generator in Fig. 3.11 are the four channel outputs. Any combination of these can be ignored in the branch metric computation by setting the corresponding erasure inputs. These erasures are needed for the decoding of punctured codes. Another use of these inputs is that in DVB mode the $J$ and $R$ inputs can be erased, allowing the use of a single BMG sub-unit for both DVB and DAB. For the DVD mode, the "BMC read in" input flag is set when reading in branch metric comparison (BMC) values to the BMG.

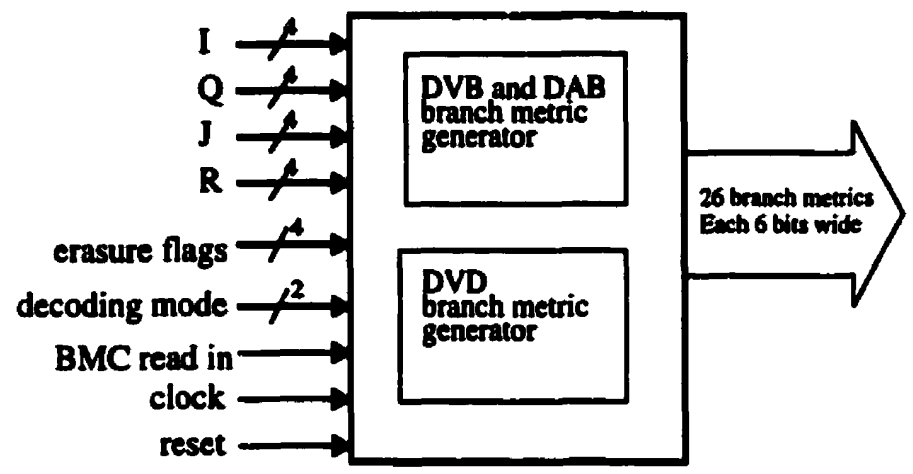

Fig. 3.11: Flexible branch metric generator

This section has described the basic types of branch metric computation performed in the BMG, along with required bit widths. More detailed implementation results are presented in Chapter 5.

\subsubsection{Add-Compare-Select Unit}

The ACS unit adds branch metrics to their corresponding path metrics, compares the sums and stores the lower path metric. Fig. 3.12 shows an "ACS cell" for state $r$. One such cell exists for each state. The path metrics (also called "state metrics") $L_{p}$ and $L_{q}$ are wired from other ACS cells according to the trellis interconnection. The corresponding branch metrics $\lambda_{p r}$ (from state $p$ to state $r$ ) and $\lambda_{q r}$ (from state $q$ to state $r$ ) are input from the BMG. Each ACS cell also stores a path metric, the output of which is routed to other ACS cells according to the trellis 
interconnection. The binary decision signal $D_{r}$ output by the comparator is forwarded to the SMU.

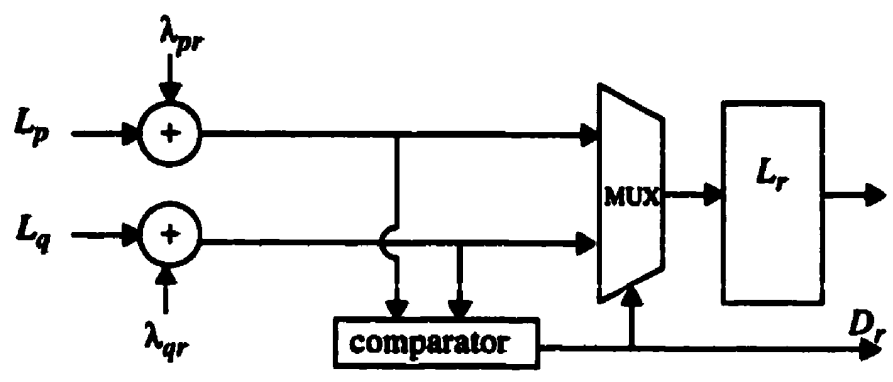

Fig. 3.12: ACS cell for state $r$

To avoid renormalization, a 2's complement subtractor is used in place of a comparator [39], and the sign-bit of the difference becomes the decision bit $D_{r}$, as shown in Fig. 3.13. The values of $S_{p}$ and $S_{q}$ are incremented modulo the bit-width of the adders, and the carry-out is ignored when the adders "roll-over". The 2's complement subtractor performs the operation: $S_{q}-S_{p}$. If $S_{q}$ is greater than $S_{p}$, the sign-bit will be 0 , indicating that $S_{p}$ should be chosen. This functionality holds even when either sum "rolls over" zero, as long as the path-metric bit-width is large enough. Twelve bits are used for path metric storage, which satisfies the minimum path metric requirement ${ }^{1}$ stated in [39]. The path metric of an ACS cell is routed to two other ACS cells based on the trellis interconnection. The decision bit $D_{r}$ is forwarded to the SMU.

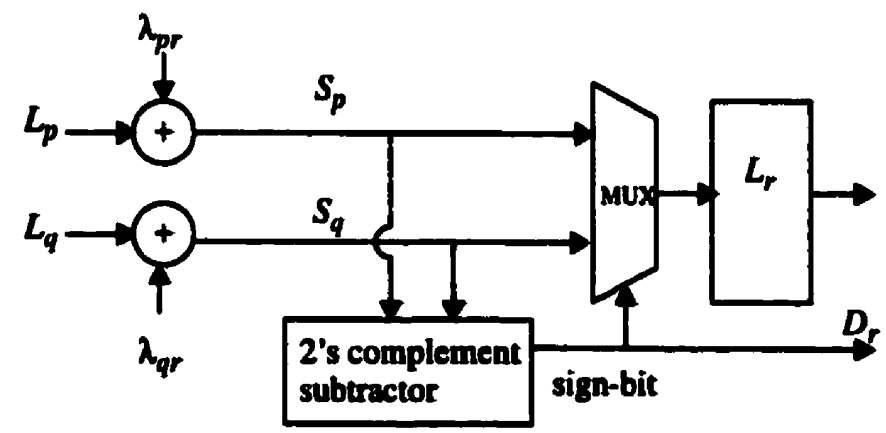

Fig. 3.13: ACS cell for state $r$ using a 2's complement subtractor to generate the decision bit

1. A lower resolution (ten bits) could have been used, based on the requirements discussed in [39]. 


\subsubsection{Survivor Memory Unit and Output Decision Device}

There are two methods for managing survivor sequences in the SMU: register exchange (RE) and traceback. As explained later, RE is chosen for implementation. A description of traceback can be found in [38], and a description of RE follows. The type of output decision device (ODD) used affects the SMU structure, and is also explained in this sub-section.

The SMU of an $n$-state VD implementing RE with survivor length $\delta$ would require $n \delta$ multiplexed registers. For a 4-state VD, Fig. 3.14 shows one stage of 4 multiplexed registers. The stages are connected based on the trellis interconnections. The decision bit $D_{i}$ from the ACS cell of state $i$ controls all $\delta$ multiplexers corresponding to that state in the SMU. On each time step, the survivors are updated based on which sequences survived the comparison. The survivor sequence for state $i$ is stored in the $i$ 'th row of registers in the SMU structure. The first stage of registers have fixed inputs: for states $i$ with $i<n / 2,0$ 's are shifted in and 1 's are shifted in to the remaining states. At the final stage, $n$ bits are shifted out to the ODD.

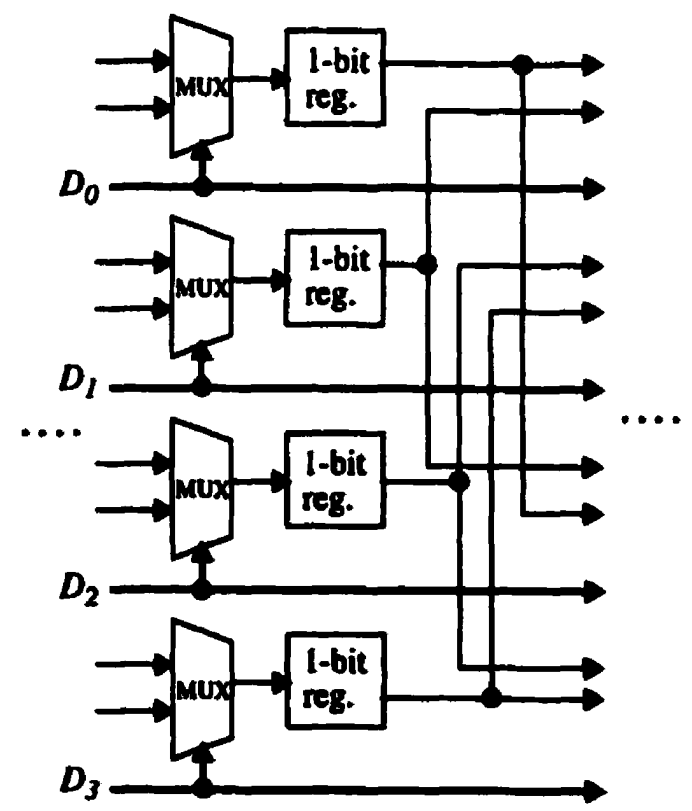

Fig. 3.14: One stage of the SMU - $\delta$ of these would be cascaded to form the SMU 
The architecture selected for the SMU is based on the register exchange (RE) technique described above. Although traceback has lower area and power dissipation (above some minimum decoder size), $R E$ has some advantages over traceback. The main advantage of $R E$ is its simplicity, which requires less time in the design and test phases. The area cost of using $R E$ instead of traceback is relatively small: it has been shown that for $\mathrm{\delta} \delta=\mathbf{7 0}$ with 64 states, traceback requires only 1.2 $\mathrm{mm}^{2}$ less than an RE implementation in a $0.35 \mu \mathrm{m}$ process [41] (the RE occupies about $5 \mathrm{~mm}^{2}$ in this case). RE can be synthesized and easily targeted to different technologies. For these reasons, the RE method is used in the multi-standard prototype VD.

The performance requirements determine the function of the ODD. The ODD selects an output bit from the final SMU stage. Two options are fixed-state decoding and best-state decoding. If $\delta$ has been chosen to be large enough to meet performance requirements with fixed-state decoding, there is no need for an ODD. A certain fixed state (eg. the 0-state output bit) of the final SMU stage is wired to the output. In best-state decoding, the bit corresponding to the state with the lowest path metric is output. Best-state decoding is obviously more complex, but the advantage is that the survivor depth can be about $1 / 2$ what is required for equivalent performance with fixedstate decoding [40].

The required "merge depth" (or "survivor length") determines the required number of SMU stages. Fig. 3.15 shows the BER rate of the VD using various SMU merge depths when 4-bit Euclidean distance branch metrics are used. Rate 7/8 is simulated since it is the code rate most affected by merge depth. The use of a fixed-state survivor length of about 112 leaves a margin of $1 \mathrm{~dB}$ for implemenation loss of the analog front-end which is adequate [20]. This verifies the theoretical requirement on survivor depth reported in [41]. If best-state decoding is used, the survivor length can be substantially reduced to 70 to obtain about the same performance, as stated in [41]. The performance of best-state decoding with a survivor length of 70 meets the rate 7/8 DVB-S specification by $1.05 \mathrm{~dB}$, as illustrated in Fig. 3.15. 


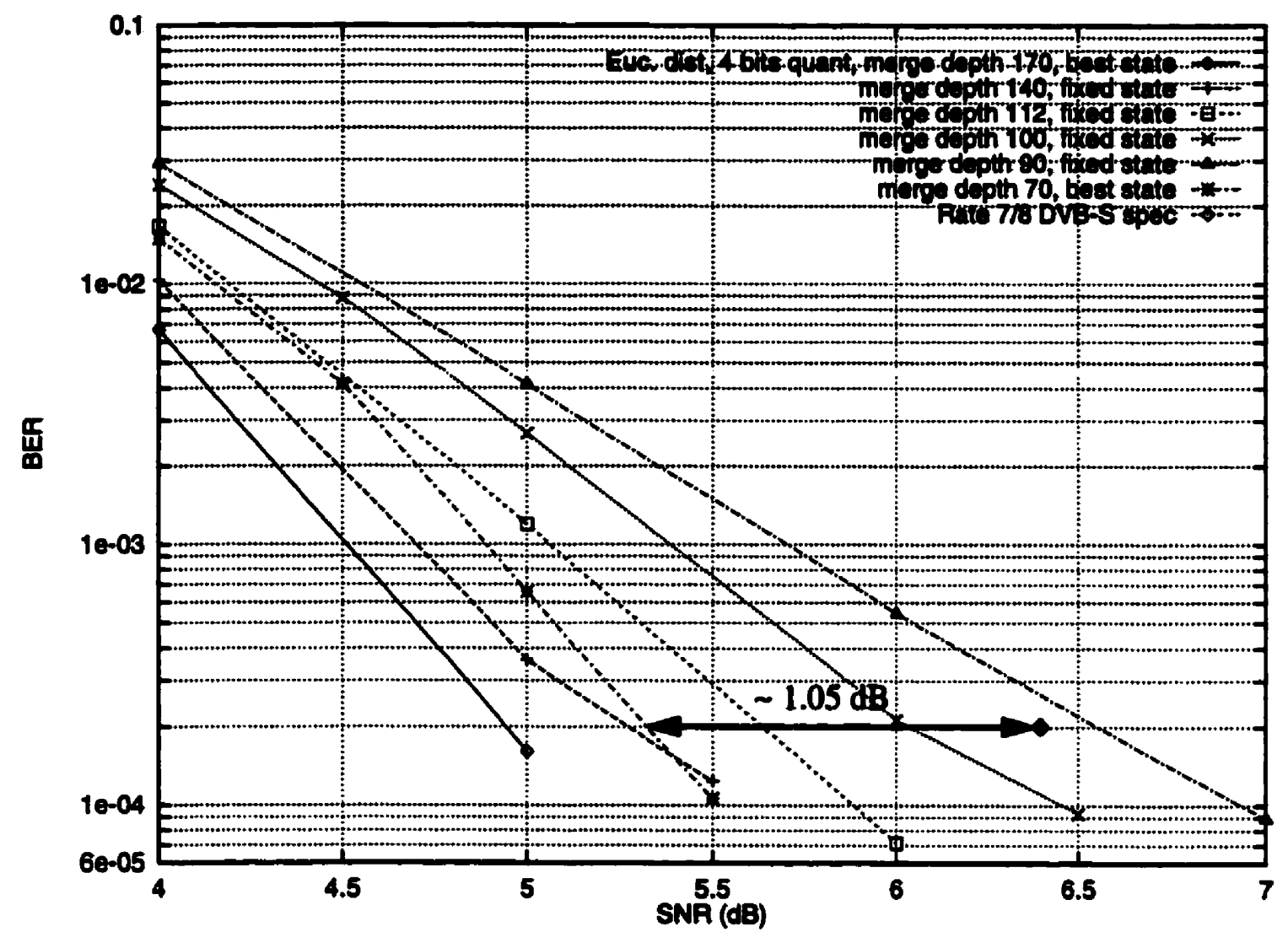

Fig. 3.15: Rate 7/8 DVB-S BER performance for various merge depths

Although using best-state decoding with a merge depth of $\mathbf{7 0}$ implies added complexity in the output decision device (ODD), 42 SMU stages can be eliminated. Synthesis of the SMU and ODD reveals that 42 stages of the SMU requires 1.5 times the cell area as the ODD for best-state decoding. Therefore, a merge depth of $\mathbf{7 0}$ with best-state decoding is selected for the SMU implementation. The ODD implementation used to determine the state with the lowest path metric is presented in Chapter 5.

\subsubsection{Accomodating Different Standards}

The DAB and DVB convolutional codes are fixed and require 64 states in the VD. However, there is flexibility in the design of the Viterbi detector for DVD, since the number of adjacent symbols which we consider to contribute to ISI can be selected. Therefore, the number of states in the 
Viterbi detector can be changed to trade off performance for complexity. As Fig. 2.19 shows, the DVD impulse response extends over several symbol periods. Removing ISI with $v=6$ gives about $0.2 \mathrm{~dB}$ gain over $v=4$ [31]. Using higher values of $v$ does not result in any significant difference in performance. Note that using $v=6$ for DVD means that the same basic 64 -state ACS and SMU structure can be used for both convolutional decoding and ISI mitigation.

As illustrated in Fig. 2.18, the EFMPlus RLL constraints imply that the NRZI waveform must have no fewer than three contiguous identical symbols. Hence, not all states and transitions are allowed. In fact, the number of states reduces from 64 to 18 , and the number of transitions reduces from 128 to 26 . The trellis representation of the allowed states and transitions is shown in Fig. 3.16.

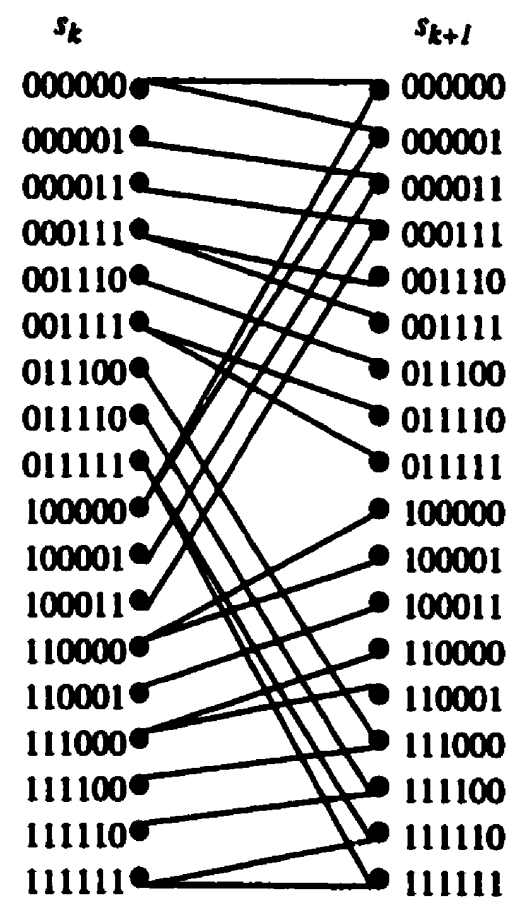

Fig. 3.16: EFMPlus constrained states and transitions

Although the valid DVD trellis interconnections are a subset of the DVB and DAB trellis interconnections, the multi-standard VD is designed to use the same ACS structure for all standards. The trellis interconnections for DVB, DAB and the unconstrained DVD trellis are identical and ACS cells are interconnected according to this trellis. In the DVB and DAB modes, 
all transitions are valid and branch metrics from the BMG are forwarded to all ACS cells. In the DVD mode, the BMG computes the branch metrics for the valid branches and forwards them to the ACS unit. The ACS unit then connects the valid branch metrics to the corresponding ACS cells and invalid branches are assigned the largest possible branch metric. Thus, in DVD mode, the ACS effectively operates according to the constrained trellis in Fig. 3.16. In states with only one valid input branch, invalid branches are not selected since they have a higher branch metric than any valid branch. Paths through the trellis containing invalid branches have higher path metrics than competing valid paths, so that survivors based on invalid branches are not output by the "best-state" ODD.

Chapter 5 presents implementation details of the VD. The depuncturing unit which processes VD input according to the code rate is discussed in the following section.

\subsection{Depuncturing Unit}

A depuncturing unit is used to process BMG inputs for the DVB-S, DVB-T and DAB standards. DVB-S and DVB-T use the same 5 code rates (shown in Table 2.1), while DAB uses 24 code rates $(8 / 32,8 / 32,8 / 30, \ldots . ., 8 / 9)$. In DVB-T, the code rate in use is transmitted on a TPS carrier (see Appendix A for details), and in DAB, the code rate is transmitted in the FIC. If in DVB-T mode, the depuncturing unit can apply erasure flags from the channel estimator. In DVB-S, the receiver must determine the code rate automatically.

In general, the depuncturing operation can be accomplished with a state machine which controls the erasure flags and VD inputs according to the depuncturing state. As an example, consider the 3/4 code rate with reference to the DVB code puncturing pattern which was shown in Table 2.1. In the single depuncturing period shown in the table, note that two pairs of $A B$ (QPSK) symbols map to three pairs of $X Y$ (VD input) symbols. Two states $D_{0}$ and $D_{1}$ exist in the depuncturing of this code rate. When $X_{1} Y_{1}$ is received, both of these inputs are passed to the VD unerased (state $D P_{0}$ ). In the next state $D P_{1}, Y_{2} X_{3}$ is received. The $X$ input to the $V D, X_{2}$, is erased (it will be ignored in branch metric computation) and the $Y_{2}$ input is passed to the VD. Next, $X_{3}$ is sent 
to the VD, with the $Y$ input erased. The state now returns to $D_{0}$ to process $X_{4} Y_{4}$ in the next depuncturing period.

A microprocessor or controller typically extracts the code rate from the received signal for DVBT and DAB. In this case, the depuncturing unit is a collection of state machines. The transmitted code rate controls which state machine to use.

For DVB-S, the condition where the VD is not operating at the same code rate as the transmitter must be detected by the receiver. One way of detecting this is monitoring the path metric growth, since path metrics will increase more quickly when the correct depuncturing pattern is not used. Re-encoding the decoded output and comparing it to the transmitted signal also gives an indication of excessive BER, which would be caused by using an incorrect code rate. However, a simple method called pseudo-syndrome synchronization [42] is used to select the code rate. Most of the cost of this method is in the calculation of the state with the minimum path metric. Recall that this state is already calculated in the ODD. This makes pseudo-syndrome synchronization well suited to the VD design.

For DVB-S, code rate selection can be modelled as a state machine which controls the receiver's code rate. Within each code rate state, another state machine controls the depuncturing state for that code rate as shown in Fig. 3.17. Pseudo-syndrome synchronization determines when to try another code rate, and when to continue using the same code rate.

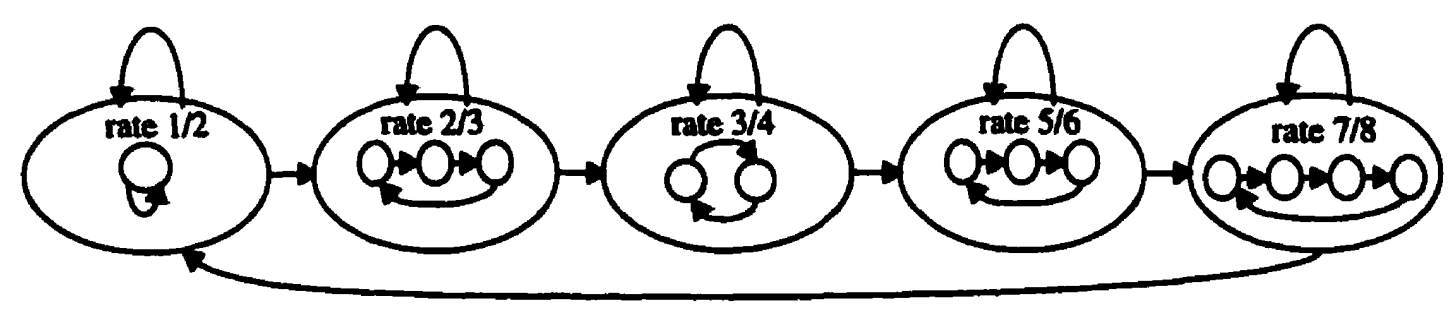

Fig. 3.17: DVB-S code rate selection and depuncturing 
In pseudo-syndrome synchronization, a value called the pseudo-syndrome (PS) is calculated from the minimum state and the channel outputs. For the DVB-S convolutional code, if we represent the minimum state at time $k$ as $S_{1, k} S_{2, k} S_{3, k} S_{4, k} S_{5, k} S_{6, k}$, then $\mathrm{PS}_{k}=X_{k+1} \oplus Y_{k+1} \oplus S_{S, k} \oplus S_{1, k}$

This value should be 0 if the VD is operating with the correct code rate, and the SNR is sufficient. The PS is observed over several symbols to obtain an estimate of the probability $P\left(P S_{k}=1\right)$. If $P\left(P_{k} \geq 0.5\right)$, the decoder is not synchronized to the transmitter's code rate and/or depuncturing state. If $P\left(P S_{k}=1\right)$ is below a certain threshold, the decoder is using the correct code rate. The PS synchronization method can be described as follows [42]:

1. If the decoder has both $X_{k+l}$ and $Y_{k+l}$ inputs available (non-erased), then increment a counter $C_{L}$ of periodicity $L$.

2. If $C_{L}$ is incremented and the $P S$ has value 1 , then increment a second counter $C_{P S}$.

3. When counter $\mathrm{C}_{\mathrm{L}}$ reaches $\mathrm{O}(\bmod L)$, compare the content $N_{P S}$ of $\mathrm{C}_{\mathrm{PS}}$ with threshold $T h<L / 2$. If $N_{P S}<T h$, then the decoder is in-synchronization. Reset $C_{L}$ and $C_{P S}$. If not in synchronization, apply the next code rate and return to step 1 .

The threshold $T h$ can be chosen based on the code rate and expected SNR. From plots of $P\left(P_{k}=\right.$ 1) for various code rates and SNRs given in [42], we select $L=1021$ and $T h=408$, or $40 \%$ of $L$. PS synchronization has been verified to acquire the correct code rate through simulations using these parameters.

Fig. 3.18 shows the high-level hardware structure of the depuncturing unit. The decoding mode input determines if PS synchronization is to be used, or whether the DAB/DVB-T code rate input should be used. The "channel state information" of DVB-T can be used to erase VD inputs which have been carried on comupted carriers. The minimum state output from the VD, and the channel 
outputs $X_{k+l}$ and $Y_{k+l}$ are used in the PS generation. The depuncturing unit design itself can be divided into combinational and synchronous logic blocks. The combinational logic determines the next depuncturing state and next code rate based on their current values and on the PS value. The $P S$ generation block contains two 10-bit counters $C_{L}$ and $C_{P S}$ and control logic which implements the PS synchronization algorithm described above. The control logic of the combinational block also determines the erasure flags and VD inputs based on the inputs, current code rate and depuncturing state values. The depuncturing unit implementation is discussed in Chapter 5.

\section{Combinational logic}

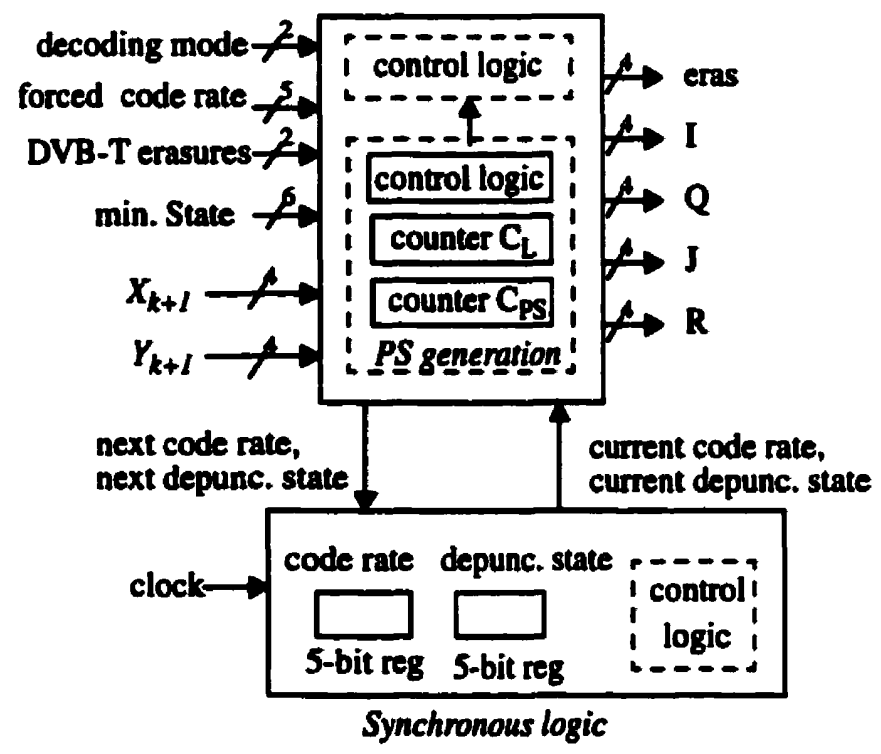

Fig. 3.18: Depuncturing Unit: high-level hardware organization 


\subsection{Summary}

In this chapter:

- The Viterbi Algorithm was introduced and the design of hardware related to the Viterbi decoder component of a multi-standard channel decoder was presented.

- Based on DVB-S and DVD simulations along with DAB and DVB-T implementations, the required parameters of a multi-standard Viterbi decoder were presented. Specifically, Euclidean distance branch metrics, 4-bit input quantization (5-bits for DVD), and a merge depth of 70 with best-state decoding should be used in the multi-standard VD. Implementation-level details will be presented in Chapter 5.

- The basic functionality and structure of a multi-standard depuncturing unit suitable to the VD design was explained, including a simple method of automatically detecting the code rate.

The next chapter presents a high-level design of a Reed-Solomon decoder, which has a very different structure from the VD. Its design is important for a multi-standard channel decoder, since DVB-S, DVB-T, DVB-C and DVD require a Reed-Solomon decoder. 


\section{Chapter 4. \\ Reed-Solomon Decoder Design}

There are two general types of error-correction codes: convolutional codes and block codes. The VD presented in the previous section operates on convolutional codes. This chapter focusses on the design of a Reed-Solomon code. Reed-Solomon codes are one of the most widely used block codes, employed in such applications as the Hubbie space telescope modem and the ubiquitous compact disc. As explained in Chapter 2, a Reed-Solomon decoder is used in DVB-S, DVB-T, DVB-C and DVD. The aim of this chapter is not to produce a complete decoder as in Chapter 3 , but to present the basic design of the components required for a RS-decoder compatible with the above standards. As explained in this chapter, a " $(255,239)$ " RS decoder (which satisfies certain requirements) could be used for the standards under consideration with appropriate inputprocessing. A high-level design of internal RS-decoder components from [44] and [45] is presented, and VHDL implementation results are presented in Chapter 5.

RS-code operations are based on symbols rather than bits. Galois field (GF) elements are the symbols in RS-decoding. The structure and operations of Galois fields are briefly explained in Appendix B. In a RS code over GF $\left(2^{m}\right)$, each symbol is an $m$-bit word. All of the standards considered which employ Reed-Solomon (RS) decoding use the same basic RS code. It operates in $G F\left(2^{8}\right)$ (also written as $G F(256)$ ), and each element in $G F\left(2^{8}\right)$ is an 8-bit symbol. Hence the symbols in the RS-decoding process for this case are bytes rather than bits.

In an $(N, I)$ RS code over GF $\left(2^{m}\right)$, the input of the block encoder is an $I$-symbol word, which is encoded into an $N$-symbol RS codeword by attaching $2 t$ redundant symbols (i.e., $N=I+2 t$ ). The error-correction capability of an (N,I) RS code is $t=(N-I) / 2$ symbols per RS codeword. The RS codes used by DVB and DVD are based on a $(255,239)$ eight-error correcting code, meaning that the $\mathrm{RS}$ code is composed of 239 information bytes and 16 redundant bytes, forming a 255-byte RS code-word. The RS-decoding algorithm and hardware descriptions in this chapter are specified in terms of the parameters $N, I$ and $t$, and for the $(255,239)$ code relevant to the standards in question, $N=255, I=239$ and $t=8$. In actuality, although the $R S$ codes for $D V B$ and 
DVD are based on the $(255,239)$ code, they are modified by shortening and/or puncturing; details will be provided in section 4.6. With the notation of RS codes established, a decoding algorithm and hardware structure is presented. The last section discusses modifications to this base design required to decode DVB and DVD RS codes.

\subsection{Reed-Solomon Decoding}

This section explains the operations which must be performed by the RS-decoder, but in the interests of brevity, the underlying theory and explanations are not included. That is, the decoder algorithm and operations are outlined here to obtain implementation complexity, area and speed estimates, but discussions and justifications of the decoder operations are given in [43] and [44]. The basic error-correcting decoder design is presented in this section, and the section 4.6 explains its application to DVB and DVD. Certain modifications required for "erasure" decoding, needed for DVD RS-decoding, are also given in section 4.6.

Two principal techniques for RS decoding are the Berlekamp-Massey algorithm [43] and a modified form of Euclid's algorithm [44]. Specifically, these algorithms are used to find the "error-locator polynomial", the roots of which indicate error positions in the RS-codeword.

The RS decoding method we use is the Modified Euclid's Algorithm (MEA) because its modularity makes it amenable to VLSI implementation. The MEA can be implemented in a timedomain decoder (TDD) or a frequency-domain decoder (FDD). For long RS codes, the area needed for the FDD can be larger than for the TDD, but for the $(255,239)$ code relevant here, the frequency domain decoder is simpler than the time-domain decoder [45]. The FDD is therefore used for the RS-decoder design. The remainder of this chapter is therefore devoted to the FDD RS-decoder design based on the MEA given in [44], with the TDD RS-decoder design outlined in Appendix C.

Fig. 4.1 shows a high-level hardware block diagram of an error-correcting frequency domain Reed-Solomon decoder. 


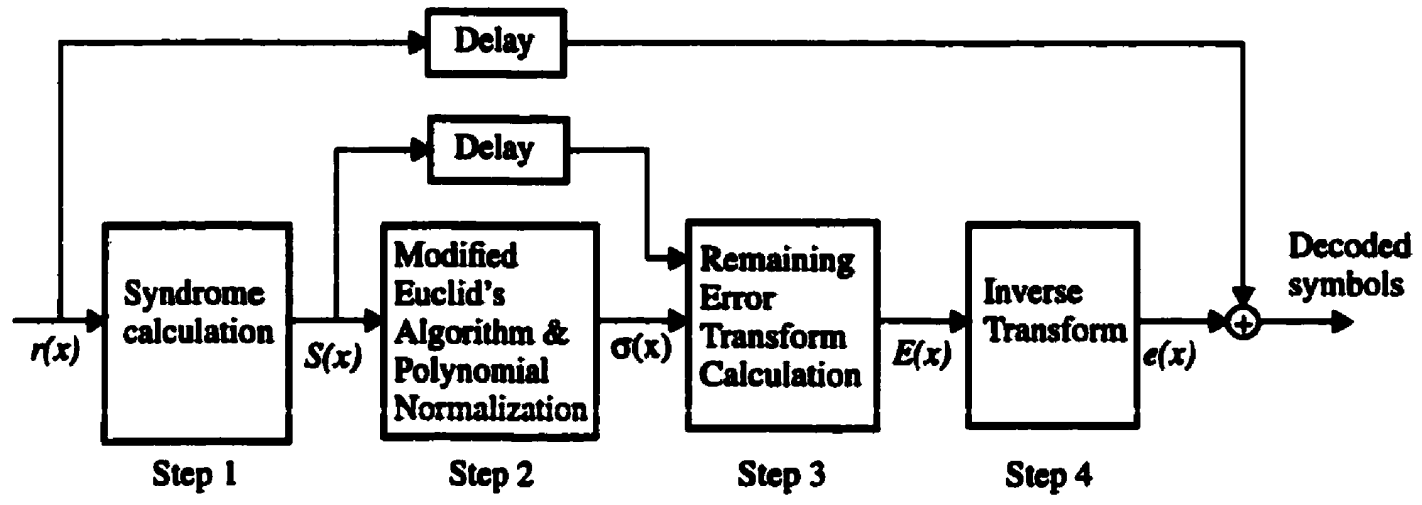

Fig. 4.1: Error correcting frequency-domain Reed-Solomon decoder using the modified Euclid algorithm

The FDD method using the MEA can be described by the following four steps, which correspond to the four main hardware blocks in Fig. 4.1:

1. Let $r_{n}(0 \leq n \leq N-1)$ be the symbols of the received RS codeword. The primitive element in $\mathrm{GF}\left(2^{8}\right)$ is denoted $\alpha$ (as in Appendix B). The first step in RS-decoding is to determine the "syndrome polynomial" $S(x)=\sum_{k=1}^{2 t} S_{k} x^{2 t-k}$, where the $S_{k}$ values are "syndrome coefficients". These are calculated by:

$$
S_{k}=\sum_{n=0}^{N-1} r_{n} \alpha^{n k}, 1 \leq k \leq 2 t
$$

The transform of the error pattern $E_{k}=S_{k}$ for $1 \leq k \leq 2 t$. The remaining elements of $E_{k}$ are calculated in step 3.

2. The MEA (described in section 4.3) is applied to the syndrome to obtain the polynomial 
$\lambda(x)=\lambda_{0} x^{t}+\lambda_{1} x^{t-1}+\ldots+\lambda_{t}$

When divided by its leading coefficient $\lambda_{0}$, the "error locator polynomial" is obtained:

$\sigma(x)=x^{t}+\sigma_{1} x^{t-1}+\ldots+\sigma_{t}$

where $\sigma_{k}=\lambda_{k} / \lambda_{0}, 1 \leq k \leq t$,

assuming $\lambda_{0} \neq 0$.

3. The elements of $E_{k}$ for $2 t+1 \leq k \leq N$ are calculated recursively:

$$
E_{2 t+j}=\sum_{k=1}^{t} \sigma_{k} E_{2 t+j-k}, \text { for } j \geq 1
$$

These samples comprise the transform of the error pattern. It is the Galois field Fourier transform of the error pattern.

4. The inverse transform of $E_{k}$ is computed to obtain the error pattern:

$$
e_{n}=\sum_{k=0}^{N-1} E_{k} \alpha^{-n k}, 0 \leq n \leq N-1
$$

Reed-Solomon codes can incorporate erasure information to improve their performance. Erasures are a crude form of soft-information on the reliability of a given symbol. A binary erasure flag is input for each symbol, to indicate if the symbol has been deemed unreliable. A t-error correcting RS decoder can correct $u$ errors and $v$ erasures provided $v+2 u \leq 2 t$. Based on the DVB and DVD systems, the Viterbi decoder which precedes the RS decoder outputs hard decisions with no reliability measures. Therefore, erasures would not be used in this type of system. However, an 
"errors and erasure" RS decoder is useful for decoding punctured RS codes (one of the DVD RS codes is punctured). There are some additional operations which must be performed for decoding with erasures which will be outlined later.

Each of the four steps in FDD, which correspond to the four major blocks in Fig. 4.1, are now explained in more detail with a view towards implementation.

\subsection{Syndrome Calculation}

Equation (3) can be rewritten as

$$
S_{k}=\left(\ldots\left(\left(\left(r_{N-1} \alpha^{k}+r_{N-2}\right) \alpha^{k}+r_{N-3}\right) \alpha^{k}+\ldots\right) \alpha^{k}+r_{0}\right)
$$

This can be computed by the structure in Fig. 4.2, where the input symbols are shifted in, starting with $r_{N-1}$. Each of the $2 t$ cells contains a GF adder and multiplier and an 8-bit register. When the input to all $2 t$ cells is $\mathrm{r}_{\mathrm{i}}$, the function of cell $k$ is $S_{k} \leftarrow r_{i}+S_{k} \alpha^{2 t+1-k}$.

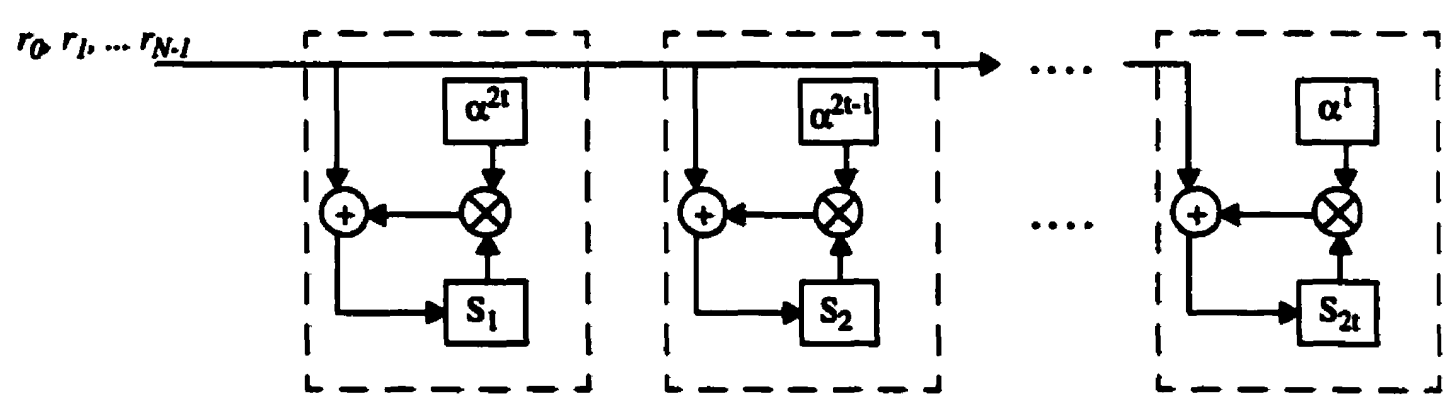

Fig. 4.2: Syndrome calculation

After all inputs are applied to the cells, the syndrome coefficients $S_{k}$ are output to the MEA block. 


\subsection{Modified Euclid's Algorithm}

The MEA is the heart of the RS-decoder, and is common to both the TDD and FDD. We start the MEA with the polynomials

$$
\begin{aligned}
& A(x)=x^{2 t} \\
& S(x)=\sum_{k=1}^{2 t} S_{k} x^{2 t-k}
\end{aligned}
$$

The MEA recursively calculates the $i$ th remainder $R_{i}(x)$, as well as $\gamma_{i}(x)$ and $\lambda_{i}(x)$ which satisfy the relation:

$\gamma_{i}(x) A(x)+\lambda_{i}(x) S(x)=R_{i}(x)$

The recursion stops when the degree of the remainder $R_{i}(x)$ becomes less than $t$. The polynomial $\lambda_{i}(x)$ is the output of interest, which corresponds to $\lambda(x)$ in (4). In the following, the notations $\operatorname{deg}(P(x))$ and $d(P(x))$ are used to represent the degree of a polynomial $P(x)$ as in [44].

The initial conditions of the algorithm are:

$$
\begin{aligned}
& R_{0}(x)=A(x) \\
& Q_{0}(x)=S(x) \\
& \lambda_{0}(x)=0 \\
& \mu_{0}(x)=1
\end{aligned}
$$

Designating $a_{i-l}$ and $b_{i-I}$ the leading coefficients of $R_{i-I}(x)$ and $Q_{i-1}(x)$, respectively, the recursion relations are: 


$$
\begin{aligned}
R_{i}(x)= & {\left[\sigma_{i-1} b_{i-1} R_{i-1}(x)+\bar{\sigma}_{i-1} a_{i-1} Q_{i-1}(x)\right] } \\
& -x^{\left|h_{i-1}\right|}\left[\sigma_{i-1} a_{i-1} Q_{i-1}(x)+\bar{\sigma}_{i-1} b_{i-1} R_{i-1}(x)\right] \\
\lambda_{i}(x)= & {\left[\sigma_{i-1} b_{i-1} \lambda_{i-1}(x)+\bar{\sigma}_{i-1} a_{i-1} \mu_{i-1}(x)\right] } \\
& -x^{\left|l_{i-1}\right|}\left[\sigma_{i-1} a_{i-1} \mu_{i-1}(x)+\bar{\sigma}_{i-1} b_{i-1} \lambda_{i-1}(x)\right] \\
Q_{i}(x)= & \sigma_{i-1} Q_{i-1}(x)+\bar{\sigma}_{i-1} R_{i-1}(x) \\
\mu_{i}(x)= & \sigma_{i-1} \mu_{i-1}(x)+\bar{\sigma}_{i-1} \lambda_{i-1}(x)
\end{aligned}
$$

where

$l_{i-1}=\operatorname{deg}\left(R_{i-1}(x)\right)-\operatorname{deg}\left(Q_{i-1}(x)\right)$

and

$\sigma_{i-1}=1$ if $l_{i-1} \geq 0$

$\sigma_{i-1}=0$ if $t_{i-1}<0$

The recursion stops when $\operatorname{deg}\left(R_{i}(x)\right)<t$.

A maximum of $2 t$ recursions are necessary to obtain the polynomial $\lambda(x)$ in (4). In a practical realization of the MEA, the operations required for each recursion are illustrated in the basic cell structure of Fig. 4.3. A total of $2 t$ of these cells could be cascaded to perform all required recursions ${ }^{1}$. A polynomial is processed by serially shifting in its coefficients, for descending powers of $x$. Logic required to control the operations in Fig. 4.3 is described by the following pseudo-code:

1. However, it is possible to reduce area by recursively using two MEA cells in the case of a $(255,239)$ code [46]. The price for the area saving is increased control logic complexity. 
Input from previous cell: $R_{i}(x), Q_{i}(x), \lambda_{i}(x), \mu_{i}(x), \operatorname{deg}\left(R_{i}\right), \operatorname{deg}\left(\boldsymbol{Q}_{i}\right)$

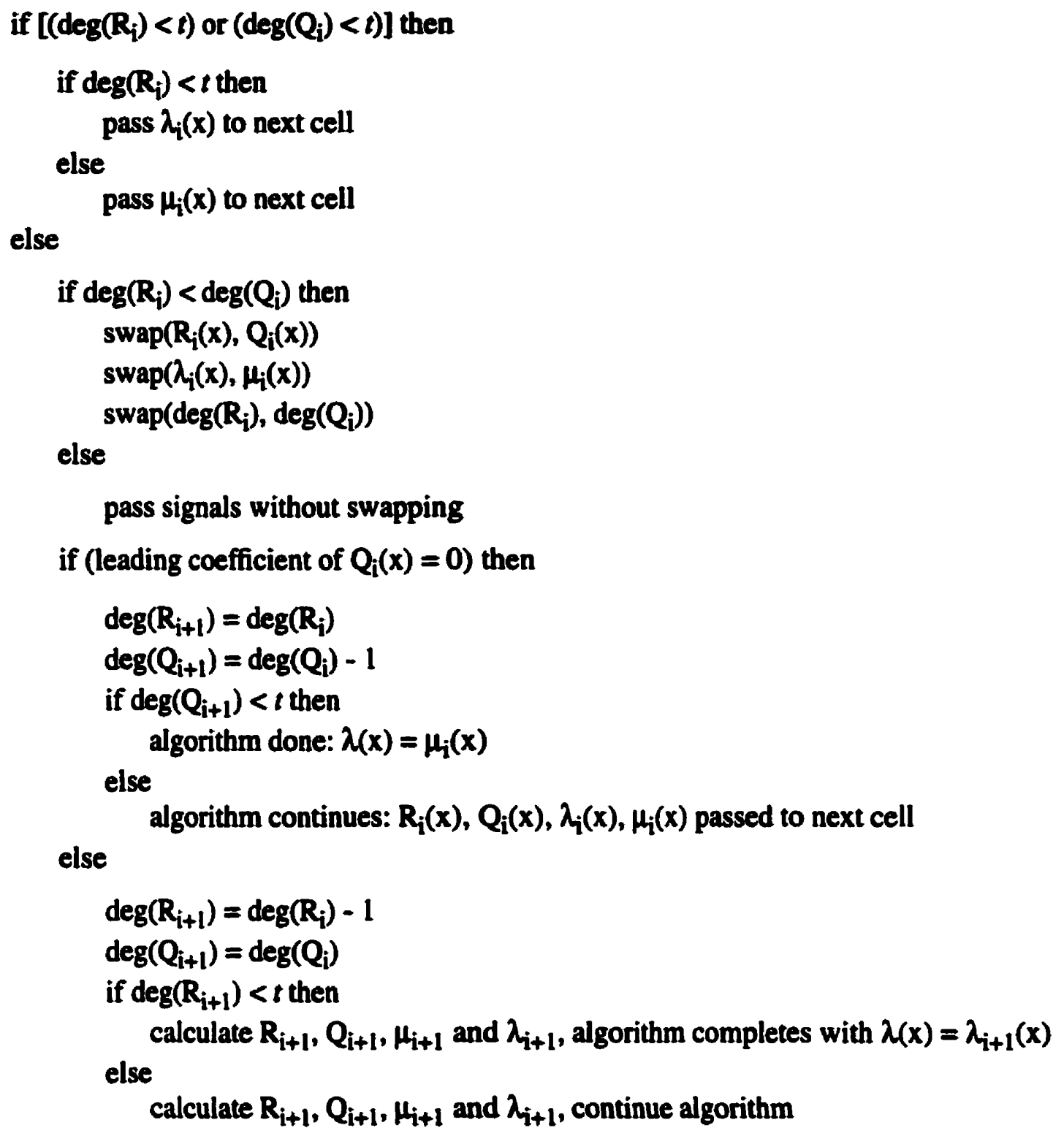




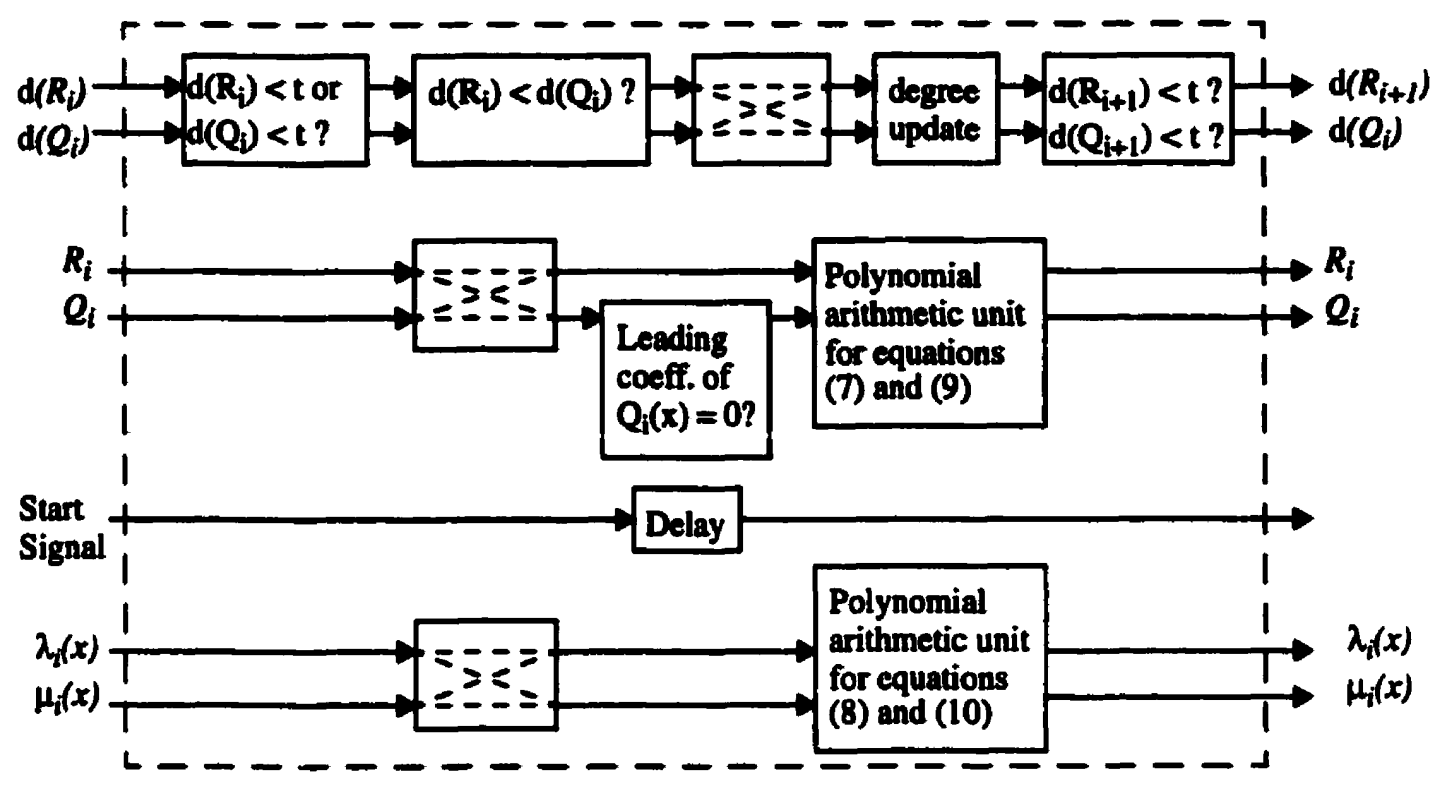

Fig. 4.3: Modified Euclid's algorithm block for the computation of one recursion

The $\lambda(x)$ output of the final MEA cell is then divided by its first nonzero element to obtain the error-locator polynomial $\sigma(x)$.

\subsection{Remaining Error Transform}

The syndromes are the first $2 t$ elements of $E_{k}$, the frequency transform of the error pattern. The remaining ( $N-2 t)$ are calculated recursively as shown in (5). A hardware model to compute the remaining error transform is shown in Fig. 4.4. After the first $2 t$ error transform coefficients (the syndromes) are shifted in, the input becomes $\mathrm{E}_{\text {acc }}$. The registers $\mathbf{R}_{\mathfrak{i}}$ contain the coefficients of $\sigma(x)$. Cells corresponding to leading zero-coefficients of $\sigma(x)$ have no delay and do not contribute to $\mathrm{E}_{\mathrm{acc}}$ as indicated by switches within the cells. The $N$ values shifted out are the frequencydomain representation of the error pattern. All that remains is to take the inverse transform and add the error pattern to the received RS codeword. 


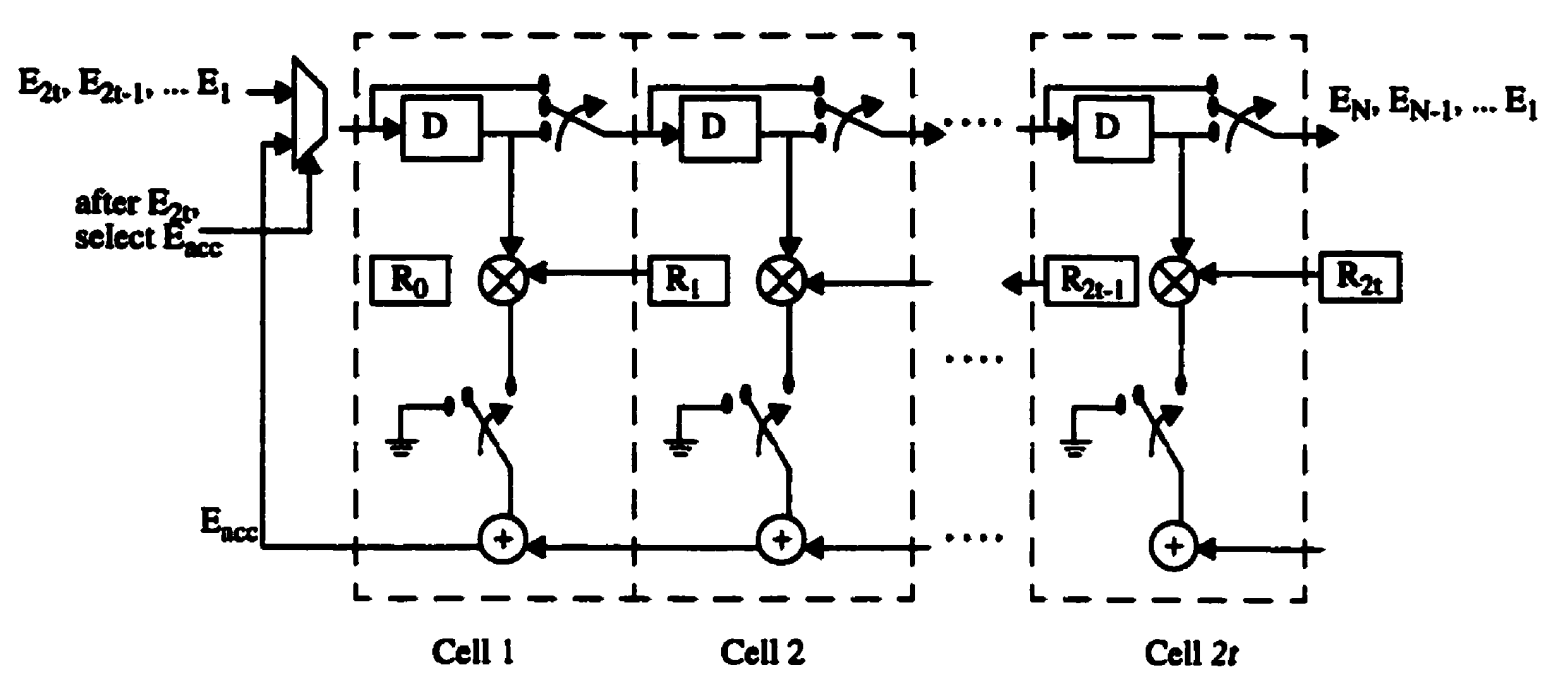

Fig. 4.4: Calculation of remaining error transform

\subsection{Inverse Error Transform}

The inverse transform of the error pattern (equation (6)) is calculated with a cell similar to that used for syndrome calculation, and is shown in Fig. 4.5. The output error pattern must be added to the received RS-codeword, which corrects at most $t$ symbol errors.

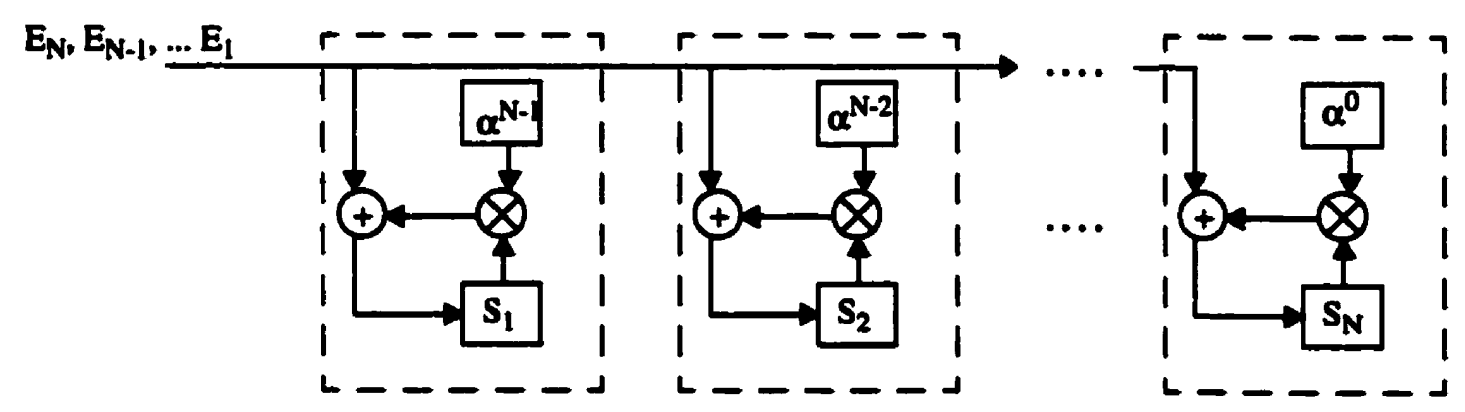

Fig. 4.5: Inverse Transform calculation

The inverse transform block consumes a relatively large area, because $\mathrm{N}=\mathbf{2 5 5}$ cells are needed for the $(255,239)$ code. Due to the shortened RS codes used, however, the area of this unit can be reduced by approximately $18-28 \%$ (based on synthesis results for $N=208, N=182$ respectively) by omitting cells for symbols which are known to be zero. These symbols are discarded after the RS-decoding operation. This component would require a maximum of $\mathbf{N}=208$ cells for one of 
the DVD RS codes.

Since the TDD method outlined in Appendix $\mathrm{C}$ does not require this component, some area could be saved in the decoder design in exchange for the higher complexity of TDD. However, use of the FDD can save time in design and test, and is suitable for the $(255,239)$ code [45]. The TDD method is useful for longer RS-codes, since its area mostly depends on $t$, whereas the FDD area mostly depends on the RS code length $N$.

\subsection{Accomodating Different Standards}

The RS-codes for DVD and DVB standards all operate in $\operatorname{GF}\left(2^{8}\right)$, and are based on a $(255,239)$ RS code. Therefore, the same GF arithmetic units and hardware can be used for different standards. However the codes are shortened and/or punctured as shown in Table 4.1. A $(N, I)$ RS code is shortened by collecting all code words for which a given coordinate is zero, and deleting that coordinate resulting in an $(N-1, I-1)$ code. The RS code specified for DVB is shortened by 51 symbols to form a $(204,188)$ code. The column RS-code of the DVD RS-PC code (explained in the next paragraph) is shortened by 47 symbols to form a $(208,192)$ code. In puncturing an $(N$, I) code, a redundant coordinate is deleted resulting in an $(N-1, I)$ code [43]. The row RS-code used in the RS-PC for DVD is shortened by 67 symbols and punctured by 6 symbols resulting in a $(182,172)$ code. These 6 symbols can be inserted and deterministically "erased" at the receiver [43]. The same basic "errors and erasure" RS-decoder can be used for all standards provided the input RS-codewords are pre-processed correctly, by zero-padding them to form the original 255byte RS-codeword. 
Table 4.1: RS parameters for DVB, DVD

\begin{tabular}{c|c|c|c|c}
\hline Standard & $(N, I)$ & $t$ & $\begin{array}{c}\text { number of } \\
\text { shortened } \\
\text { symbols }\end{array}$ & $\begin{array}{c}\text { number of } \\
\text { punctured } \\
\text { symbois }\end{array}$ \\
\hline DVB & $(204,188)$ & 8 & 51 & 0 \\
\hline DVD, row & $(182,172)$ & 5 & 67 & 6 \\
\hline DVD, column & $(208,192)$ & 8 & 47 & 0 \\
\hline
\end{tabular}

Each frame of the DVD RS-PC code is of the form shown in Fig. 4.6. Recall that since symbols are taken from $\mathrm{GF}\left(2^{8}\right)$, they are represented as 8-bit bytes. Each block of $192 \times 172$ bytes is encoded by a $(208,192)$ RS code in the column direction, then by a $(182,172)$ RS code in the row direction resulting in a frame of $208 \times 182$ bytes. Therefore, the rows are decoded first, resulting in a $208 \times 172$ byte block which is processed by a column RS-decoder.

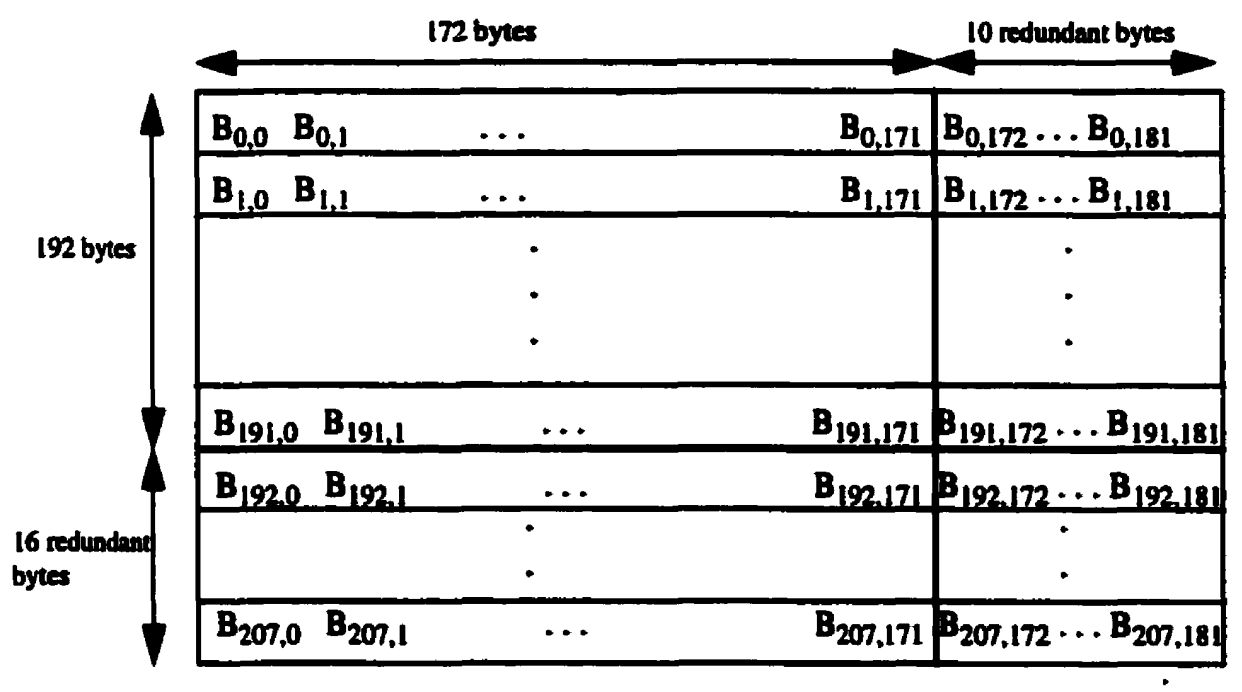

Fig. 4.6: DVD RS-PC frame structure [49]

A symbol can be labelled "erased" at the RS-decoder input if it has been determined unreliable by the preceding block (eg. Viterbi decoder or demodulator). In the case of the $(182,172)$ row RS code, 6 redundant symbols have been punctured (deleted) at the transmitter. At the receiver, 
therefore, 6 dummy symbols are inserted and these 6 positions are labelled erased. Certain modifications are required to the basic error-correcting RS-decoder of section 4.1 to correct erasures. A block diagram of the modified design (adapted from [45], [46]) is given in Fig. 4.7.

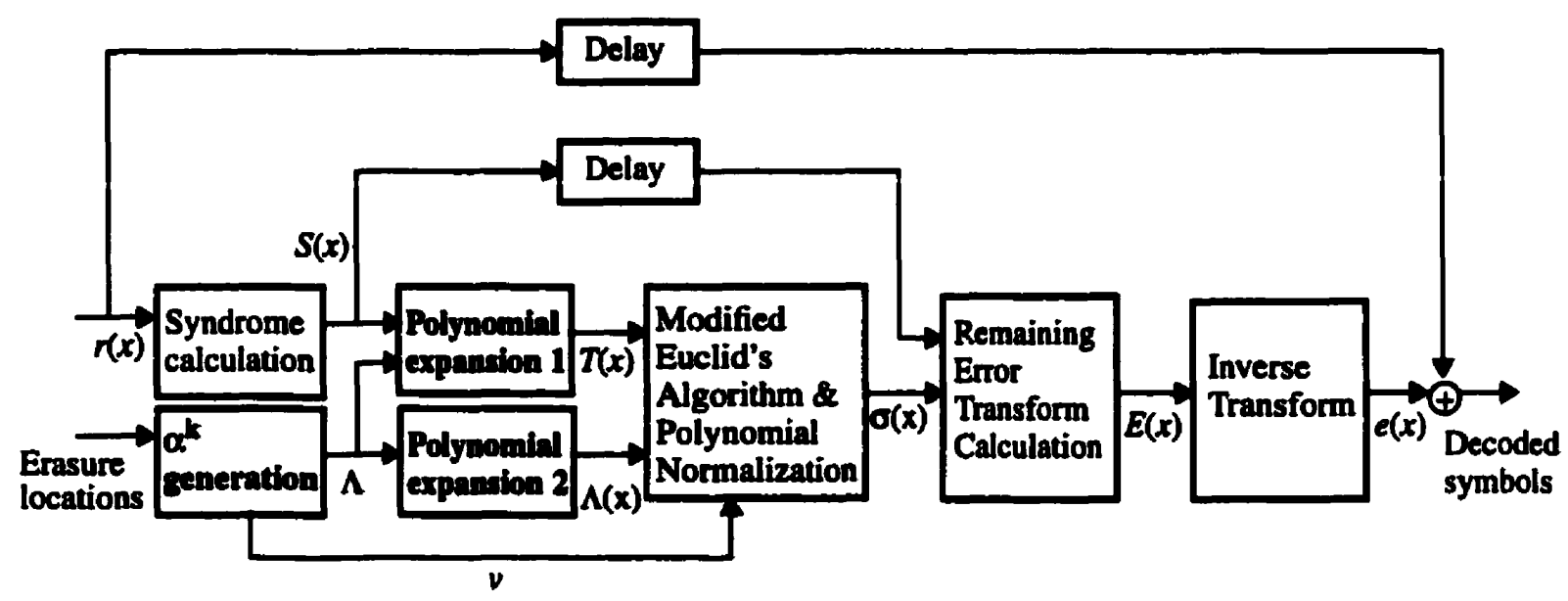

Fig. 4.7: Errors and erasure RS decoder

Erasure information is typically received in the form of a binary sequence synchronous with the received message. If symbol $r_{i}$ is erased, the block labelled " $\alpha$ " generation" outputs $\alpha^{-i}$. These outputs form a sequence of symbols $X_{j}$ which are processed by the block labelled "Polynomial expansion 2" to calculate the erasure locator polynomial, $\Lambda(x)$. If the number of erasures is denoted $v$, the erasure locator polynomial is defined as $\Lambda(x)=\prod_{j=1}^{\nu}\left(1-X_{j} x\right)$.

The "Forney syndrome" polynomial $T(x)$ is defined as $T(x) \equiv S(x) \Lambda(x) \bmod x^{d-1}$ and is computed by the block "Polynomial expansion 1".

An architecture for " $\alpha{ }^{k}$ generation" is presented in Appendix D, which is simpler than the architecture specified in [45]. The architecture of the polynomial expansion blocks (similar to the syndrome computation) is also given in Appendix D; see [45] and [46] for further details. 
For decoding errors and erasures, the MEA is modified so that $\mu_{0}(x)$ is initialized to $\Lambda(x)$ instead of 1 , and $Q_{d}(x)$ is initialized to $T(x)$ instead of $S(x)$. The error-correcting MEA stops when the $\operatorname{deg}\left(R_{i}\right)<t$ or $\operatorname{deg}\left(Q_{i}\right)<t$. In the errors-and-erasures decoder, this is generalized so that $\operatorname{deg}\left(R_{i}\right)$ or $\operatorname{deg}\left(Q_{i}\right)$ must be $\leq\left\lfloor\frac{2 t-2+v}{2}\right\rfloor$ for the MEA to finish.

\subsection{Summary}

In this chapter:

- A short introduction to Reed-Solomon codes and a Reed-Solomon decoder architecture has been presented, with Galois fields briefly explained in Appendix B.

- The parameters of DVB and DVD RS codes have been presented, to select a single architecture which can decode them.

- It was shown that a RS decoder which handles erasures is required for DVD. Since soft-information is not typically passed from the Viterbi decoder to the RS decoder in DVB, current DVB RS decoders typically correct only errors and not erasures. This type of RS decoder could not be used for DVD RS codes since the $(182,172)$ code is punctured. Therefore, a RS decoder which corrects errors and erasures must used to handle both DVB and DVD. 


\section{Chapter 5. \\ Viterbi and Reed-Solomon Decoder Implementations}

The design of the Viterbi decoder (VD) was presented in Chapter 3 and the Reed-Solomon (RS) decoder design was presented in Chapter 4. This chapter gives final implementation results for the decoders. A silicon implementation of the VD was produced, and the RS decoder components were synthesized using VHDL.

\subsection{Viterbi Decoder}

\subsubsection{Chip Architecture}

Fig. 5.1 shows the high level chip architecture based on the design discussed in Chapter 3. Each of these component implementations is now discussed in turn.

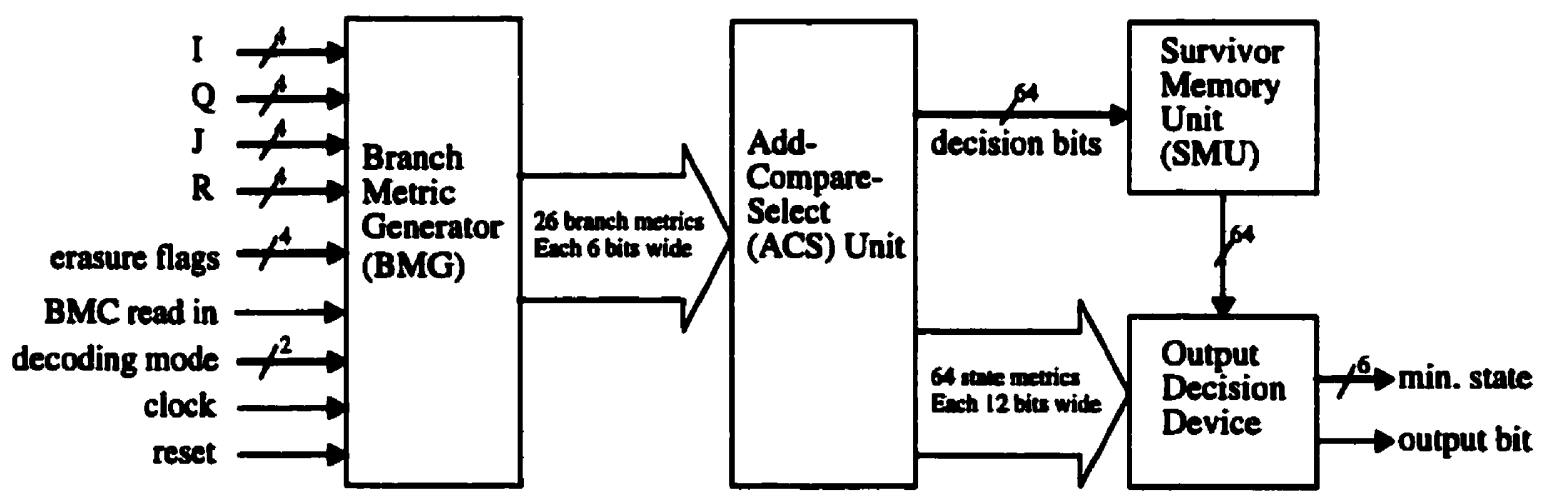

Fig. 5.1: Overall chip architecture

A description of the branch metric generator (BMG) and its inputs was given in section 3.2.1. The sub-blocks of Fig. 3.11 are now explained. The DVD sub-block of the BMG calculates the difference between channel outputs and expected transition values for the 26 valid transitions. The calculation of branch metrics for DAB and DVB are handled by a BMG sub-block composed 
of 16 cells of the form of Fig. 5.2. The cell inputs $I, Q, J, R$ and the four erasure inputs $I_{\text {eras, }} Q_{\text {eras, }}$, $\mathbf{J}_{\text {eras, }}$ and $\mathbf{R}_{\text {eras }}$ correspond to inputs shown in Fig. 5.1. Each cell is associated with a different four bit binary number denoted by digits abcd. Sixteen of these cells calculate all possible branch metrics for DAB, as there are sixteen possible expected branch values (four DAB input symbols per branch). In DVB mode, the $J_{\text {eras }}$ and $R_{\text {eras }}$ inputs are set to ' 1 ', so that only $I$ and $Q$ contribute to the branch metric computation.

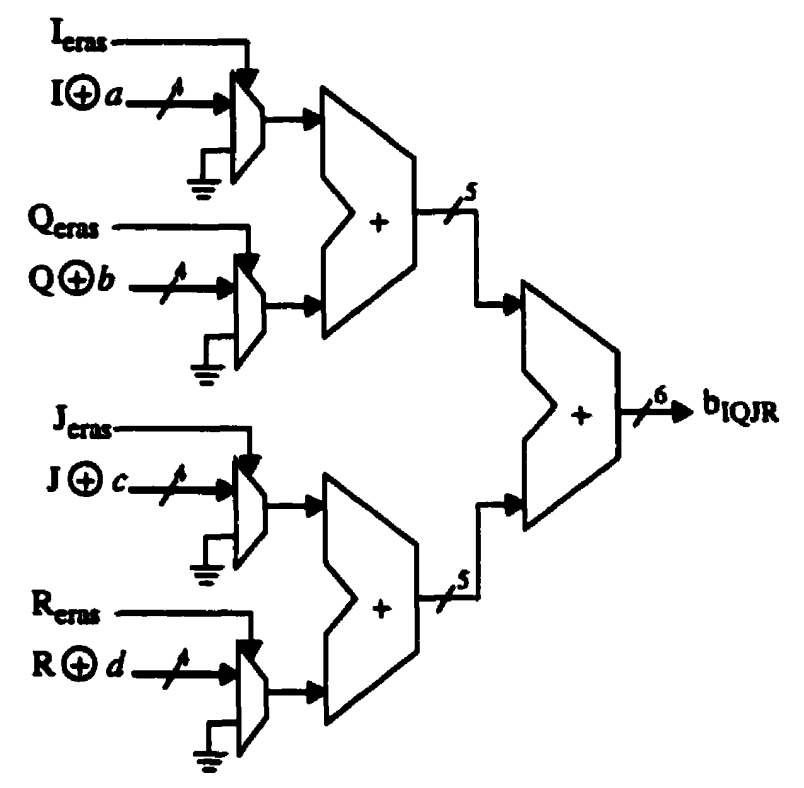

Fig. 5.2: DVB and DAB branch metric calculation for cell number iqjr

As explained in section 3.2.1, DAB requires the highest number of bits per branch metric ( 6 bits) for a total of 16 branch metrics. Compared to DVB and DAB, DVD requires the maximum number of branch metrics to forward to the ACS unit. In DVD mode, the 5-bit input sample at any given time must be compared to the 26 expected values, generating twenty-six 5-bit branch metrics. These expected values are read in and stored, allowing for the VD to be used on different DVD systems. Fig. 5.3 shows the timing of control signals to read in the 26 branch metric comparison (BMC) values for DVD. On the clock cycle following the synchronous reset, normal VD operation begins with the BMC values read in. The 5-bit BMC samples are input using the same pins used for DVD input samples: the four bits of the I input and the MSB of the $Q$ input. 


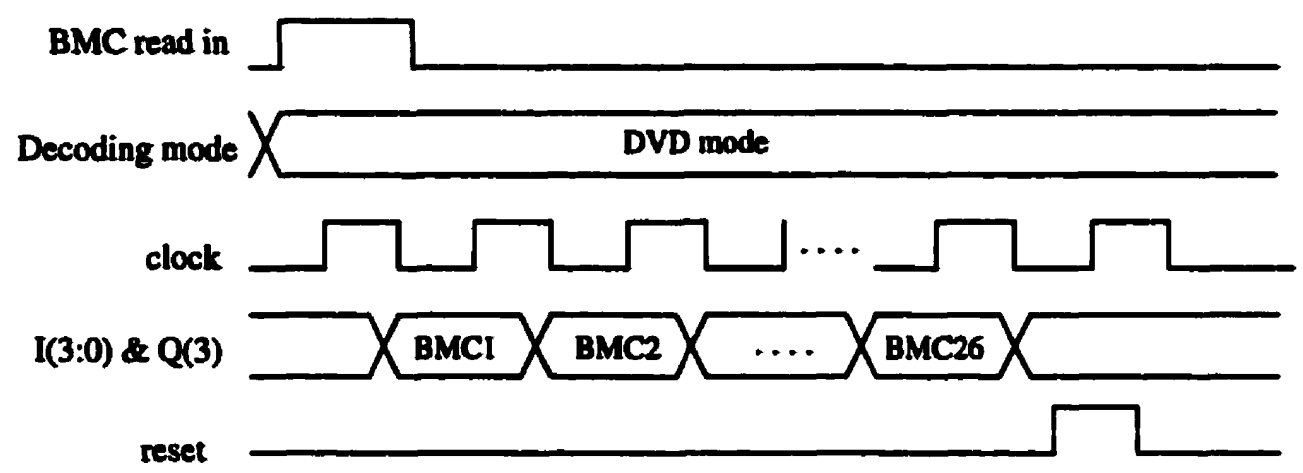

Fig. 5.3: Reading in Branch Metric Comparison (BMC) values

Erasure flags are provided for handling multiple code rates, enabling any combination of the four inputs to be ignored. The mode (DAB, DVB, or DVD) requires a two-bit input. An input flag is used to indicate when branch metric comparison (BMC) values for DVD are to be read. A clock and reset pin are also supplied to the BMG. Its outputs are 26 branch metrics which are used in DVD mode (only 4 are actually used in DVB mode, 16 in DAB mode), each 6-bits wide.

The ACS unit is composed of 64 ACS sub-units, with the $r$ th such sub-unit illustrated in Fig. 5.4. Each ACS cell is of the form in Fig. 3.13. In Fig. 5.4, a zero is prepended to the 5-bit DVB and DVD branch metrics, making all branch metrics 6-bits. DVD branch metrics corresponding to valid branches are routed from the BMG whereas invalid branches are assigned a 6-bit branch metric of " 111111 ". Since the maximum valid DVD branch metric is " 011111 ", an invalid branch will never be selected. 


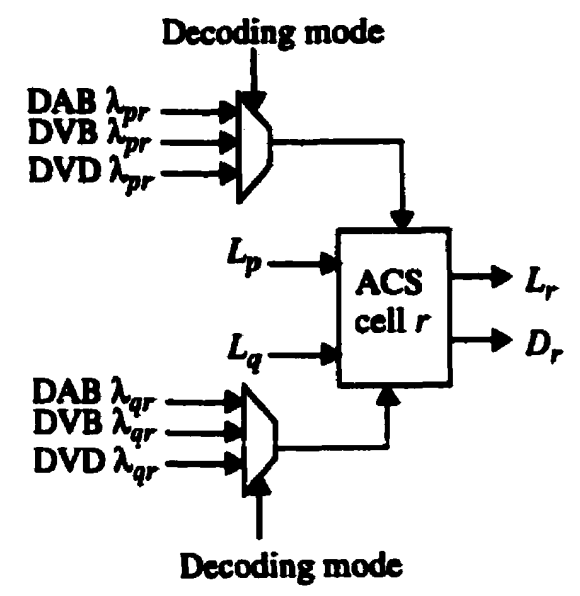

Fig. 5.4: ACS sub-unit

The survivor memory unit (SMU) is of the form given in Fig. 3.14, with 64 states, and 70 stages cascaded sequentially. Its output is forwarded to the output decision device (ODD) which must select the minimum state metric, determine the corresponding state number, and output the corresponding bit from the SMU. As shown in Fig. 5.1, the minimum state is output from the ODD along with the output bit. Recall that the minimum state is required for pseudo-syndrome synchronization in the depuncturing unit. A basic ODD cell which performs a "compare-select" operation on the first two state metrics has the form shown in Fig. 5.5. Each cell determines the minimum state metric and corresponding state number.

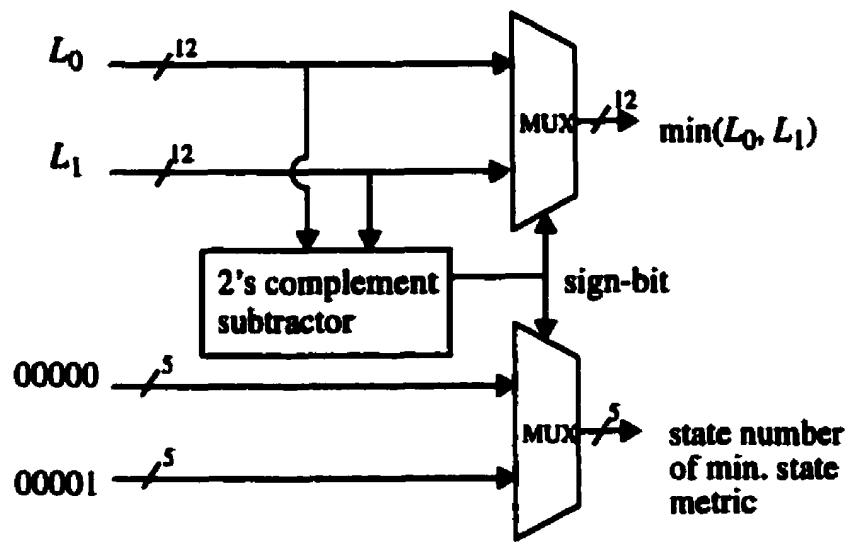

Fig. 5.5: ODD cell 1 , row 1

The high level structure of the ODD is shown in Fig. 5.6. The 64 state metries from the ACS unit 
and their corresponding state numbers are input to the first row which outputs 32 state metrics. The process continues until only two state metrics remain, input to row 6 . The minimum overall state metric is calculated, and the ODD cell in row 6 outputs the state with the minimum state metric, and selects the corresponding output bit from the survivor memory unit.

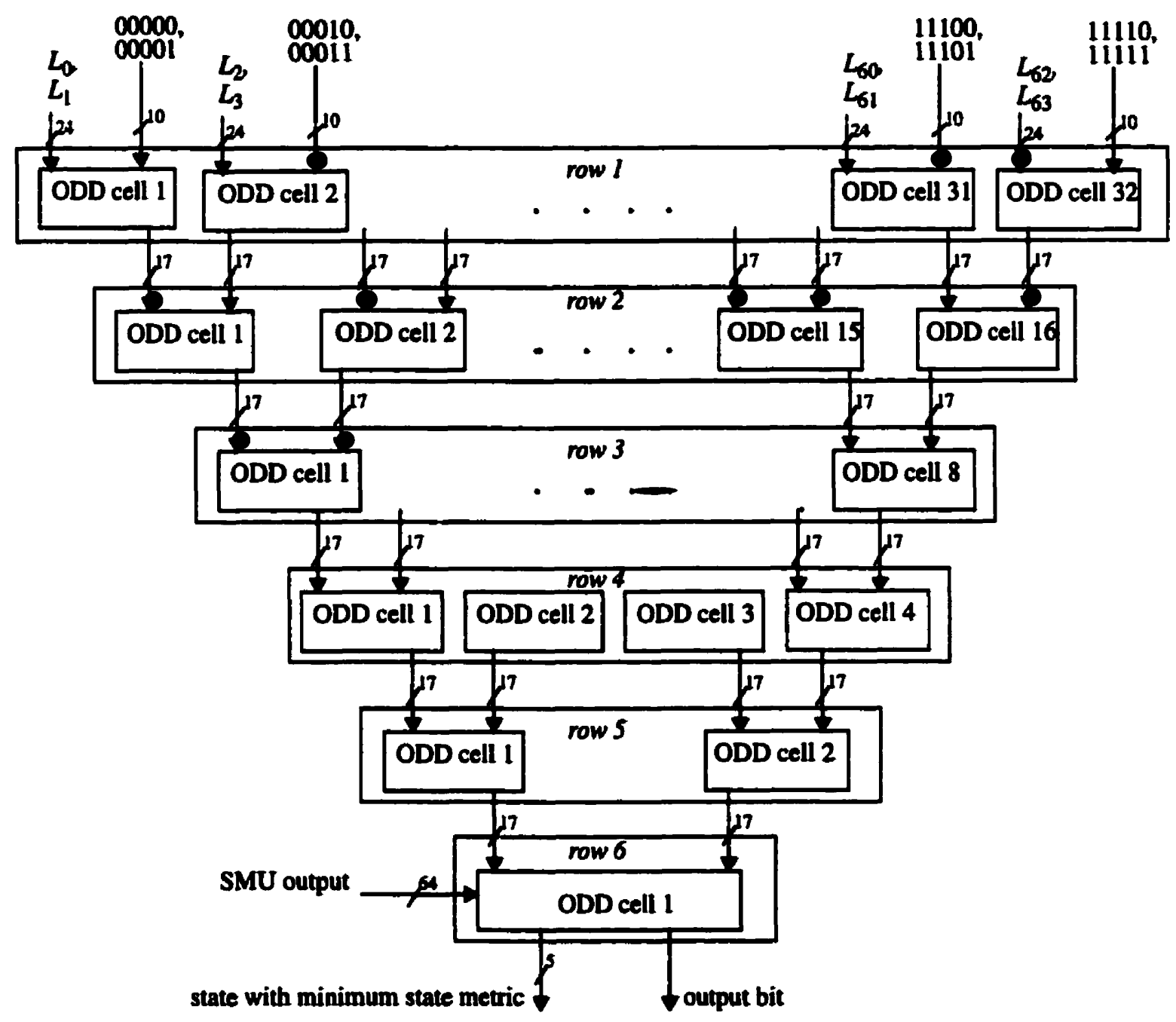

Fig. 5.6: ODD high level structure

\subsubsection{Implementation Results}

The VD design was initially tested through $\mathrm{C}$ simulations. Based on these simulations, the VD was implemented in VHDL, and tested with gate-level simulations. The VD was implemented in a three-metal layer $0.35 \mu \mathrm{m}$ CMOS technology. The chip specifications are shown in Table 5.1. 
The VD maximum output bit-rate of 62.5 Mbps meets or exceeds some existing DVB Viterbi decoder implementations (e.g., [50], [51], [52], [53], [54]).

Table 5.1: Chip characteristics

\begin{tabular}{l|l}
\hline \multicolumn{1}{c|}{ Parameter } & \multicolumn{1}{c}{ Value } \\
\hline Process & TSMC 0.35 $\mu \mathrm{m}$ CMOS 3LM \\
\hline Core area & $25.18 \mathrm{~mm}^{2}$ \\
\hline Total area & $48.89 \mathrm{~mm}^{2}$ \\
\hline Supply voltage & $3.3 \mathrm{~V}$ \\
\hline Pin count & 57 total (35 $\mathrm{VO}, 22$ power/ground) \\
\hline Packaging & $84-\mathrm{PGA}$ \\
\hline Speed & $62.5 \mathrm{Mbps}$ \\
\hline Power & approx. $1.40 \mathrm{~W}$ at an operating voltage of $3 \mathrm{~V}$ \\
\hline
\end{tabular}

The chip layout is shown in Fig. 5.7, and the pin descriptions are given in Table 5.2.

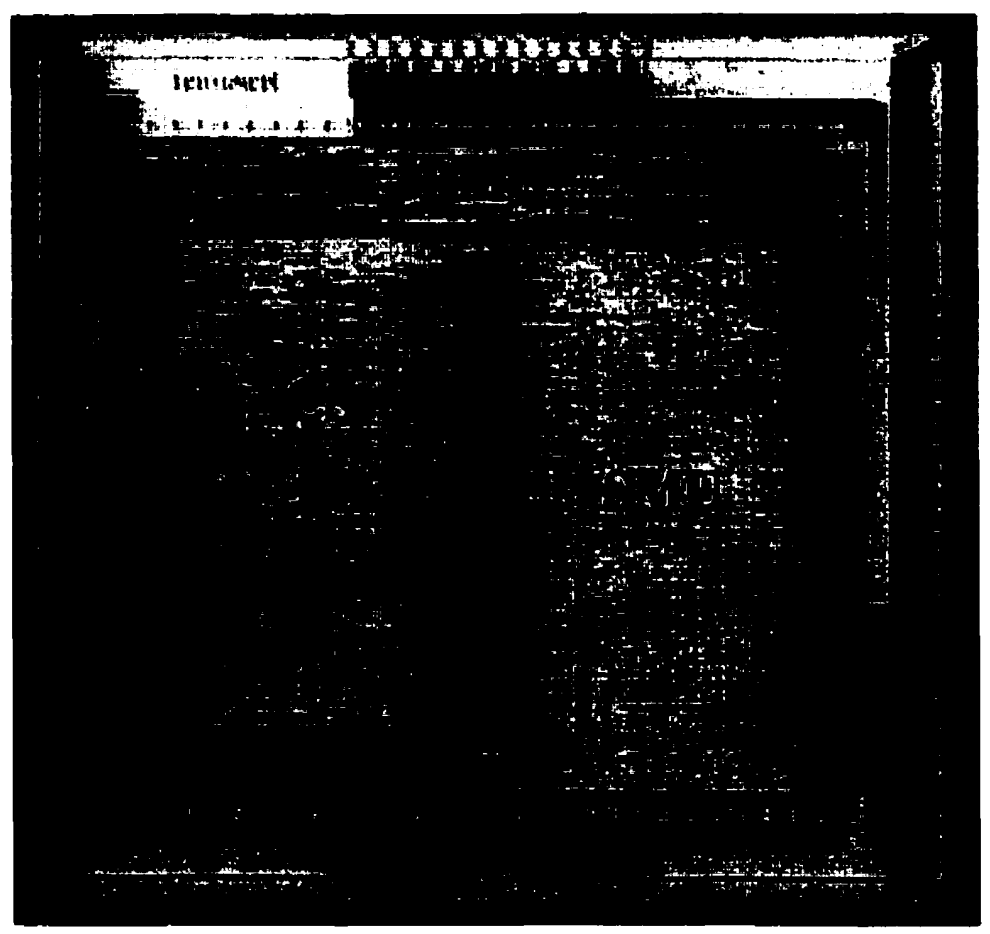

Fig. 5.7: Viterbi decoder chip layout 
Table 5.2: VO Pin Descriptions

\begin{tabular}{l|l}
\hline \multicolumn{1}{c|}{ Pin } & \multicolumn{1}{c}{ Description } \\
\hline Dmode[0:1] & Mode of operation. DAB =00, DVB =01, DVD =01. \\
\hline IQJRin[0:15] & Four inputs I, Q, J and R, each four bits wide. \\
\hline bmsReadin & $\begin{array}{l}\text { Flag indicating that branch metric comparison values are } \\
\text { to be read in. }\end{array}$ \\
\hline clk & Clock pin \\
\hline reset & Global reset \\
\hline eras[0:3] & Erasure flags, one for each input I, $Q, J$ and $R$. \\
\hline SE & Scan enable (for testing) \\
\hline SI & Scan in (for testing) \\
\hline SO & Scan out (for testing) \\
\hline minState[0:5] & Outputs the state with the minimum path metric \\
\hline outBit & VD final output bit \\
\hline
\end{tabular}

The relative area cost with respect to a VD dedicated to each standard can be estimated by scaling down the area of certain components. We estimate that $50 \%$ of the BMG area could be removed, and $10 \%$ of the ACS area could be removed for dedicated DVB and DAB receivers. Based on a scaling of the components of the implemented VD, we estimate that a VD with dedicated components would require roughly $19 \mathrm{~mm}^{2}$ of core area and a DVD receiver with only 18 states would require approximately $2.5 \mathrm{~mm}^{2}$ of core area. A crude approximation based on existing DVB decoder component implemenations [55], [56], [57], implies that an optimized (possibly full-custom layout), dedicated Viterbi decoder for DVB would consume $-0.5 \mathrm{~W}$ of power.

A depuncturing unit was also tested in $\mathbf{C}$ and specified in VHDL. The pseudo-syndrome (PS) synchronization technique discussed in Chapter 3 is implemented to lock onto the code rate at the transmitter. The depuncturing unit processes inputs to the VD, controlling its erasure flags based on the code rate and depuncturing state. In addition to the DVB code rates, the DAB 
depuncturing operation for one code rate (2/3) is implemented. The other 23 DAB code rates could be implemented in the same way. Since the DAB code rate is transmitted in the FIC, no automatic code rate acquisition is required for DAB. Due to the low computational complexity of the PS technique, the synthesized depuncturing unit is capable of operating at $200 \mathrm{MHz}$ with a cell area of less than $0.09 \mathrm{~mm}^{2}$ in a $0.35 \mu \mathrm{m}$ CMOS process.

\subsubsection{Test}

As shown in Table 5.2, the chip contains scan in (SI), scan enable (SE) and scan out (SO) pins. During synthesis, all latches were replaced with multiplexed flip-flops to allow full controllability of the VD registers during testing. The path metrics (or the contents of any register) could be read out through the SO pin. This test circuitry can be used to check for manufacturing faults.

\subsubsection{Critical Path}

The critical path is from the BMG to a path metric storage register in the ACS. This limits the speed of the chip to $62.5 \mathrm{MHz}$. This could be increased to exceed 78.8 $\mathrm{MHz}$ (the speed of one of the fastest available implementations ${ }^{1}$ [19]) by using a faster ACS sub-unit (as in Fig. 3.13) since the current implementation has a ripple-carry delay. Since the chip fabrication, an ACS sub-unit has been synthesized which operates at over $140 \mathrm{Mbps}$, as explained in Appendix E.

1. In the receiver in question, $45 \mathrm{Msymbols/sec} \mathrm{data} \mathrm{rates} \mathrm{are} \mathrm{handled.} \mathrm{Since} \mathrm{two} \mathrm{bits} \mathrm{(one} \mathrm{QPSK} \mathrm{symbol)}$ are input to the VD at a time, at rate $7 / 8$ (see Table 2.1), the VD speed must be at least 45 Msymbols/sec $*(7 / 4)=78.8 \mathrm{Msymbols} / \mathrm{sec}$. 


\subsection{Reed-Solomon Decoder}

\subsubsection{Architecture}

The architecture of a flexible Reed-Solomon (RS) decoder presented in this section is based on the "errors and erasure" RS decoder architecture presented in section 4.6. This decoder must be preceeded by a "de-shortening unit" which would insert either 51,47 or 67 null symbols depending on the type of RS codeword it is processing (see Table 4.1). In the case of the punctured DVD code, 6 extra null symbols would be added, and marked as erased.

A high-level structure of the RS decoder is presented in Fig. 5.8, based on [49]. The RS decoder corrects errors and erasures. Each RAM is 208 by 182 bytes, corresponding to the RS-PC frame structure. In this implementation, each RAM fulfulls the function of the RS codeword delay line in Fig. 4.7. The need for dual input buffers is explained in the next section. Consider an example of this decoder's operation in DVD mode where the incoming RS row codeword $R S C W_{1}$ is decoded by the RS decoder, and is stored in RAM1. The error pattern $e$ is used to correct the stored RS codeword as the next row RS codeword, $\mathrm{RSCW}_{2}$ is processed. When all rows of the first RS-PC frame have been read into RAM1 and decoded, the input rows are directed to RAM2 and decoded. Rows of RAM2 and columns of RAM1 are processed alternately by the RS decoder providing its throughput rate is high enough. When all rows of RAM2 have been decoded, its columns are decoded as the following RS-PC frame rows are read into RAM1. The decoded columns are sequentially output as the RS-PC output. This structure could also be used for DVB, using a single row of a single RAM. 


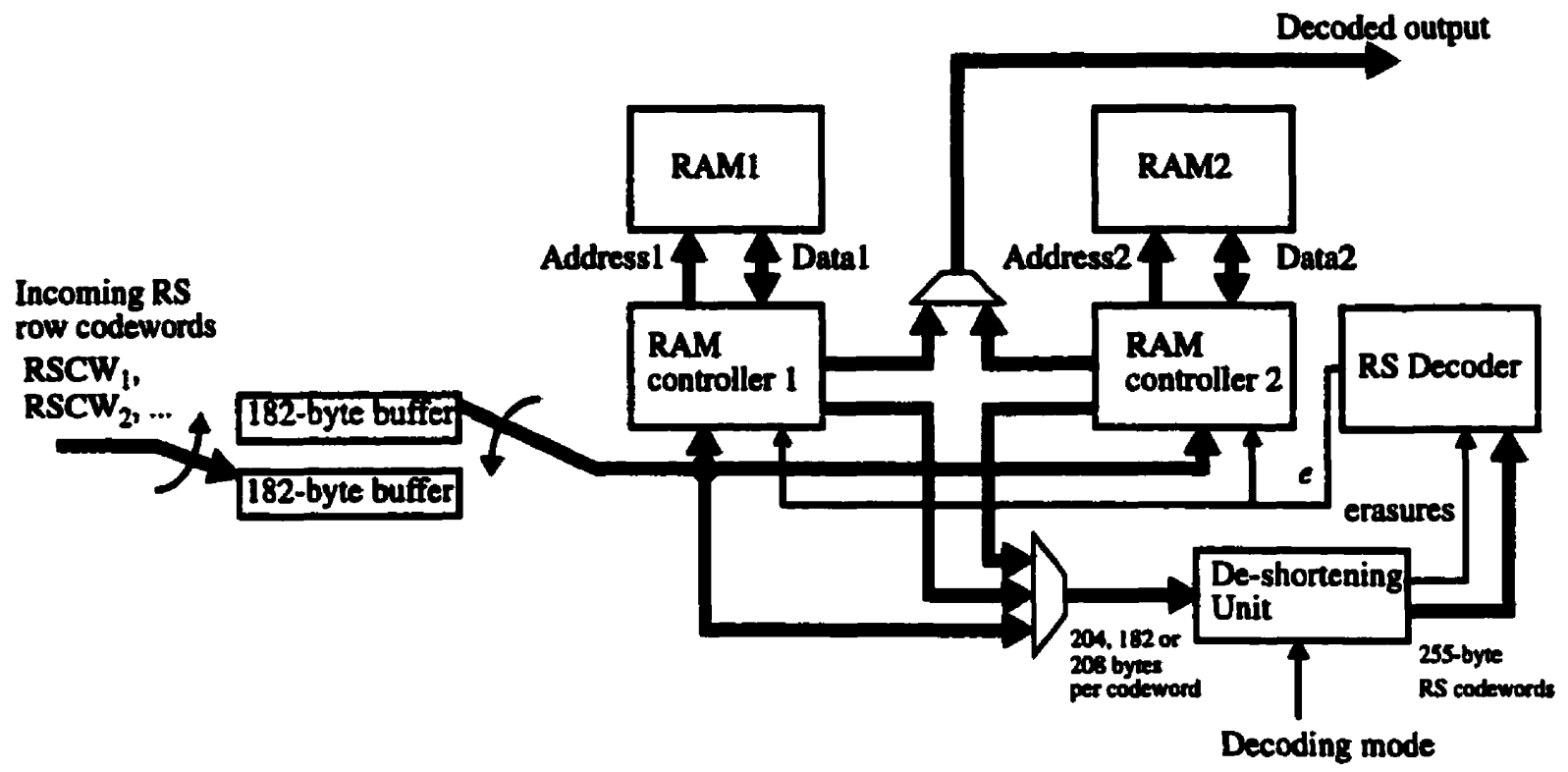

Fig. 5.8: RS-PC decoder structure

The errors and erasure RS decoder VHDL implementation results are presented in the next section.

\subsubsection{Implementation Results}

The complete FDD based RS decoder design was functionally tested in Matlab simulations. The components of the RS decoder outlined in Chapter 4 were synthesized to obtain area and speed estimates. The Galois field multiplier and inverter design used in the RS decoder components are based on [48]. An important parameter for implementation is the required RS decoder speed. There is no maximum speed stated in the DVB-S specification, but if the VD input symbol rate is assumed to be as high as $80 \mathrm{Msymbols} / \mathrm{sec}$, the VD output would be $80 \mathrm{Mbps}$. Since the 8-bits which make up RS code symbols are input in parallel, the data rate into the RS decoder is $10 \mathrm{MB}$ / sec. Since the RS decoder processes one symbol per clock period, this corresponds to a clock rate of $10 \mathrm{MHz}$.

In the case of DVD at IX, the data rate after the VD is $27 \mathrm{Mbps}$ which is decreased to $13.5 \mathrm{Mbps}$ 
after the 16/8 EFMPlus decoder (which maps 16 channel bits to 8 information bits). Again, there are 8 bits per RS codeword symbol and the RS input symbol rate becomes $1.688 \mathrm{MB} / \mathrm{sec}$. If two RS decoders are used for decoding rows and columns of RS-PC frames (as is commonly done), this implies $1.688 \mathrm{MHz}$ since one symbol is processed per clock cycle. At $8 \mathrm{X}$, the required RS decoding speed would be $13.5 \mathrm{MHz}$.

The architecture presented in Fig. 5.8 uses only one RS decoder. To determine the required RS decoder operating frequency, consider the following scenario. A 182-byte row RS codeword has just been read into an input buffer, and the following code word is about to be read into the other input buffer. The RS-decoder must decode both a row and a column by the time the following complete 182-byte RS row codeword is read in. Denoting the input symbol rate of the RS row codewords $\boldsymbol{R}_{\text {row }}$ (in units of bytes/second), the time available for the RS decoder to decode both a row and a column is $182 / R_{\text {row }}$ seconds. There are a total of 510 bytes in a row and column, so the required RS decoder speed is $\frac{510}{182} R_{\text {row }}$ bytes/second (or $\mathrm{MHz}$, since one byte is processed per clock cycle). For IX DVD this implies a required RS decoder speed of $4.73 \mathrm{MHz}$ and at $8 \mathrm{X}$, a required speed of $37.84 \mathrm{MHz}$.

Since the design of the $\mathbf{R S}$ decoder components are pipelined, most have similar critical paths (through a GF multiplier and adder). Based on synthesis results for all RS decoder compontents, the critical path is in the MEA cell. Specifically, the critical path is from the $b_{i-1}$ register through a GF multiplier and adder, to the $\lambda_{i}$ output (this operation is based on equation (8)).

The RS decoder components described in section 4.6 were synthesized from VHDL descriptions using fully parallel GF arithmetic units, resulting in an implementation which runs at $100 \mathrm{MHz}$ (which could handle 20X DVD data rates). The cell areas of the synthesized blocks are given in Table 5.3, based on the same $0.35 \mu \mathrm{m}$ technology used in the VD implementation. These should be considered lower bounds as control logic and interconnecting circuitry would also be required. 
Table 5.3: Estimated area of RS decoder components using parallel GF arithmetic units

\begin{tabular}{c|c}
\hline Component & Cell area $\left(\mathrm{mm}^{2}\right)$ \\
\hline Syndrome calculation & 0.32 \\
\hline$\alpha^{\mathrm{k}}$ generation & 0.02 \\
\hline Forney syndrome calculation & 0.37 \\
\hline Polynomial expansion & 0.49 \\
\hline Modified Euclid's Algorithm & 2.38 \\
\hline Remaining Error Transform & 0.30 \\
\hline Inverse Transform (208 cells) & 3.99 \\
\hline Delays (implemented with flip-flops) & 0.39 \\
\hline Total & $-8.26 \mathrm{~mm}^{2}$ \\
\hline
\end{tabular}

The functionality of the components could be preserved and the RS decoder speed could be reduced by time-sharing GF arithmetic units, which would results in a more area-efficient solution. The number of GF multipliers and adders required can be reduced by half, resulting in an operating speed of $50 \mathrm{MHz}$ which satisfies the $8 \mathrm{X}$ DVD requirement. Based on synthesis results, the total cell area could thus be reduced by $2.00 \mathrm{~mm}^{2}$, resulting in a total cell area of approximately $6.26 \mathrm{~mm}^{2}$.

If a lower DVD speed is being targeted, the level of time-sharing could be increased by using still fewer GF arithmetic units. However, a high-speed RS-decoder would be useful for "picture-inpicture" digital television systems where multiple streams (eg., DVB-S and DVB-C) are decoded simultaneously.

\subsubsection{Test}

In a chip implementation of the RS decoder, the same multiplexed flip-flop scan methodology 
used for the VD could be used. The labelled signal lines in Fig. $4.7(S(x), T(x)$, etc.) would be the most important signais to control and observe through scan pins to allow testing of sub-blocks.

\subsection{De-interleaver}

Although the de-interleaver design is not considered in this thesis, some general observations can be made from Fig. 2.23. The DAB and DVB-T de-interleavers process inputs before their corresponding depuncturing units, and could be merged. The DAB and DVB-T specifications indicate the specific de-interleaving patterns to use [4], [2]. Fig. 5.9 shows a high-level deinterleaver architecture. Assuming the de-interleaver input is read into a RAM, the sequence of addresses to read data out from could be stored in a ROM. That is, the ROM contents could control the RAM locations to read/write from. Different ROMs could be used depending on which standard is in use. This method could also be used for the DVB byte-convolutional deinterleaver, or it could be implemented as a bank of FIFOs, as in Fig. 2.7.

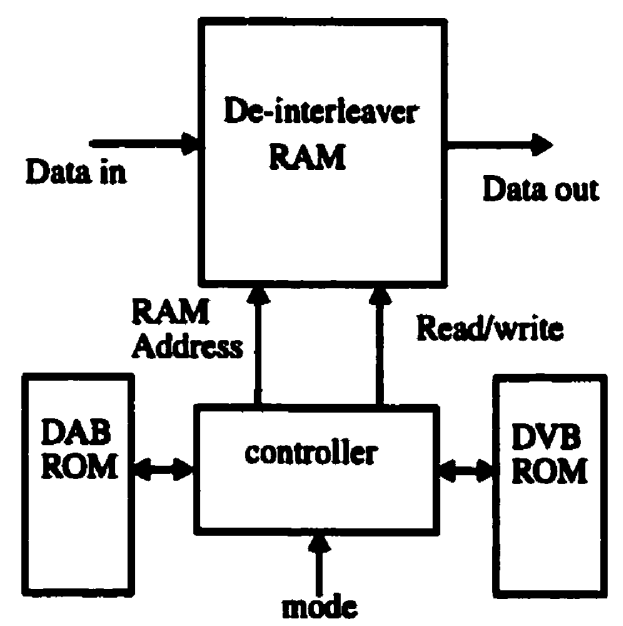

Fig. 5.9: Basic de-interleaver structure 


\subsection{Summary}

In this chapter:

- The architecture and implementation results of a flexible Viterbi decoder which operates with DVB, DAB and DVD standards was presented. The Viterbi decoder can generate branch metrics appropriate to the different standards, and the 18 ACS units required for DVD equalization are implemented using the same hardware as needed for DAB and DVB.

- A flexible depuncturing unit which can operate according to the DVB and DAB standards has been implemented (a single DAB puncturing pattern is implemented, and all other DAB code rates could be similarly handled based on the puncturing patterns in [4]).

- A high-level RS decoder architecture was given which can decode DVB and DVD signals. The components of a RS decoder were synthesized and implementation results presented. The decoder implementation allows for a simple method of trading speed for area by timemultiplexing GF arithmetic units. 


\section{Chapter 6. Conclusions}

\subsection{Summary and Conclusions}

Consumer electronics for home entertainment is a rapidly growing market. Digital services to the home are specified in standards. By taking advantage of similarities between different standards, unified receiver components can be created to reduce overall cost. Chapter 2 presented an overview of the systems considered. It was shown that the Viterbi decoder and Reed-Solomon decoder were common to most of the standards. Chapter 3 presented a Viterbi decoder which operated with all of the relevant standards. An overview of the Reed-Solomon decoder architecture was given in Chapter 4. Finally, implementation results for the VD and synthesis results for the RS decoder were presented in Chapter 5.

An examination of the processing requirements of a set-top box justifies the need for hardware implementation of the decoders. For example, the implemented Viterbi decoder performs 4 billion ACS computations per second. With time-shared Galois field arithmetic units, the ReedSolomon decoder performs 7000 GF multiplications and additions per second. Until software based decoders can achieve the speed required for these computations, hardware-based decoding is necessary.

This thesis has dealt primarily with a selected set of open standards for emerging digital services. There are many other services which have similar receiver architectures which could be combined. One example of this is Internet access via cable. A cable modem receiver is similar to a DVB-C receiver, and contains a Reed-Solomon decoder. The high-speed achievable by the RS decoder design presented in this thesis could be appropriate for cable modems and high-speed receivers for future services. There are many possible receiver combinations; this thesis has focussed on a set of standards which are suitable for a home entertainment set-top box with broad appeal. 


\subsection{Contributions}

Chapter 2 clearly showed the common channel decoding elements among DVB, DAB and DVD standards. To the author's knowledge, this is the first unified presentation of these channel decoding operations presented in the literature.

Chapters 3 and 5 presented the design and implementation of a Viterbi decoder which can be used for convolutional decoding of DVB and DAB which had also not yet been explained. The application of Viterbi detectors to DVD readout systems has only very recently been proposed. To the author's knowledge, no single Viterbi decoder implementation which handles more than one of these standards has yet been published. The decoding of the DVB and DAB convolutional codes along with ISI reduction in DVD makes the VD design one of the most unique and useful for near-future set-top-boxes.

In Chapter 4, the parameters of RS codes used in DVB and DVD were organized, and a specific architecture was selected which handles these standards. Based on an analysis of the RS code requirements, it was determined that a RS decoder which handles errors and erasures must be used in a unified RS decoder design. A specific design based on the modified Euclid's algorithm was presented and implemented, showing area and speed characteristics in Chapter 5. This would be useful for an implementation of all channel decoding components on a single chip.

\subsection{Future Work}

The Viterbi and Reed-Solomon channel decoders were the focus of this work as they were the primary baseband algorithms common to all considered standards. There are many possible areas of future work to create a single receiver which could perform all necessary functions for the standards described. Some examples of this are de-interleaving and frame synchronization. The demodulation functions could be examined and potentially combined into a single multi-standard demodulator. The RF front end and the timing/carrier recovery functions are other possibilities for convergence. 
Currently, ISI mitigation performed by the flexible Viterbi decoder in DVD mode uses only 18 of the 64 states available. If the ACS and SMU interconnections were made programmable, it would be possible to remove a higher degree of ISI from the DVD readout signal. This could enable the use of enhanced DVD discs which have a higher data density than regular DVD discs. A DVD player equipped with such a Viterbi detector could playback regular DVDs as well as enhanced DVDs.

Another useful area of investigation would be the use of a soft-output Viterbi decoder. This could take the form of the Soft Output Viterbi Algorithm [58] (SOVA), or an erasure-declaring VD [59]. Soft-output from the VD could provide erasure information to the RS-decoder. Iterative decoding could also be used, where the RS decoder results are used to correct errors in the VD [60]. Iterative decoding concepts can also find applicability to turbo coding in the context of digital video broadcasting, which could provide $1.6 \mathrm{~dB}$ gain over the performance of the DVB standard system [61]. 


\section{References}

[1] ETSI standard, "Digital Video Broadcasting (DVB); Framing structure, channel coding and modulation for 11/12 GHz satellite services", EN 300 421, 1997

[2] ETSI standard, "Digital Video Broadcasting (DVB); Framing structure, channel coding and modulation for digital terrestrial television", EN 300744,1997

[3] ETSI standard, "Digital Video Broadcasting (DVB); Framing structure, channel coding and modulation for cable systems", EN 300 429, 1998

[4] ETSI standard, "Digital Audio Broadcasting (DAB) to Mobile, Portable and Fixed Receivers", ETS 300 401, 2nd ed, 1997

[5] Digital Video Broadcasting Project, http://www.dvb.org/dvb_framer.htm, 1999

[6] World DAB Forum, http://www.worlddab.org/index.html, 1999.

[7] USA Digital Radio, http://www.usadr.com/USADRTechnology/what_is_iboc_dab.htm, 1999

[8] DVD Faq, http://www.sel.sony.com/SEL/consumer/dvd/about_faq.html, 1999

[9] M. Genoe, P. Delforge, E. Schutz, "Experience of IP-Reuse in System-on-Chip Design for ADSL", IEEE Intermational Solid-State Circuits Conference (ISSCC) Digest of Technical Papers, 1999, WA 21.1, p. 360-361.

[10] Sharewave, Inc., http://www.sharewave.com/, 1999.

[11] MorphICs Technology, Inc., http://www.morphics.com/, 1999.

[12] PS2web.com, “Playstation 2 Technical Specifications", http://www.ps2web.com/hardware/ specs.shtml, 1999.

[13] VLSI Technology, "Design Reuse Cuts Time to Market", http://www.vlsi.com/products/ design.shtml, 1999.

[14] 4i2i Communications Ltd., http://www.4i2i.com/, 1999

[15] M. Miller, B. Vucetic, L. Berry (editors), Satellite Communications, Kluwer Academic Publishers, 1993.

[16] A. Romanowski, O. Klank, K. Fazel, "Concept of a Multi-standard Receiver for Digital Broadcast and Communication Services", IEEE Transactions on Consumer Electronics, vol. 43, no. 3, Aug. 1997 
[17] A. Menkhoff, M. Harteneck, M. Kindler, P. Schollhorn, "Performance of an Advanced Receiver Chip for DVB-S and DSS", IEEE Transactions on Consumer Electronics, vol. 45, No. 3, Aug. 1999, p. $965-969$

[18] M. Cominetti, V. Mignone, A. Morello, M. Visintin, "The European System for Digital Multi-Programme Television by Satellite", IEEE Transactions on Broadcasting, vol. 41, no. 2, June 1995.

[19] A. Kwentus, S. Jaffe, S. Tsai, et al, "A Single-Chip Universal Digital Satellite Receiver with $480 \mathrm{MHz}$ IF input", vol. 42, IEEE International Solid-State Circuits Conference (ISSCC), 1999, p. 332-333.

[20] H. Meyr, M. Moeneclaey, S. Fechtel, Digital Communication Receivers, Wiley, 1998, p. 533578.

[21] ETSI standard, "Digital Video Broadcasting (DVB); Implementation guidelines for DVB terrestrial services; Transmission aspects", TR 101 190, 1997

[22] M. Jacklin, P. MacAvock, A. Oliphant, "Facts about DVB-T", http://www.dvb.org/dvb_articles/dvb_pcml10.htm\#_Toc437081532, 1999

[23] C. Del Toso, P. Combelles, J. Galbrun, et al, " $0.5 \mu \mathrm{m}$ CMOS Circuits for Demodulation and Decoding of an OFDM-Based Digital TV Signal Conforming to the European DVB-T Standard", IEEE Journal of Solid-State Circuits, vol. 33, no. 11, Nov. 1998.

[24] M. Tognin, L. Soyer, P. Combelles, C. Del Toso, et al, “A VLSI Solution for a Digital Terrestrial TV (DVB-T) Receiver', International Broadcasting Convention IBC '97, 1997, p. 343-348.

[25] D. Bryan, "QAM for Terrestrial and Cable Transmission", IEEE Transactions on Consumer Electronics, vol. 41, no. 3, August 1995, p. 383-391.

[26] M. Haas, F. Kuttner, T. Todytek, et al, "Flexible Two IC Chipset for DVB on Cable Reception", IEEE Transactions on Consumer Electronics, vol. 42, no. 3, August 1996, p. 335340.

[27] G. Drury, "Digital Video Broadcasting on Cable Television Media", IEE Colloquium on DVB (Digital Video Broadcasting), Digest 1995/142, p. 2/1-2/6.

[28] L. Tan, J. Putnam, F. Lu, et. al. "A 70Mb/s Variable-Rate 1024-QAM Cable Receiver IC with Integrated $10 \mathrm{~b} A D C$ and FEC decoder", IEEE International Solid-State Circuits Conference (ISSCC), 1998, p. 200-201.

[29] K. Yamauchi, S. Kakiuchi, A. Takebe, M. Sugitomo, "Digital Audio Broadcasting Receiver Development", Intermational Broadcasting Convention IBC '95, 1995, p. 71-75 
[30] L. Thibault, M. Le, "Performance Evaluation of COFDM for Digital Audio Broadcasting, Part I: Parametric Study", IEEE Transactions on Broadcasting, vol. 43, no. 1, Mar. 1997.

[31] K. Chalmers, "A CMOS DVD 4x Viterbi Detector: System Design and VLSI Implementation", MASc thesis, University of Toronto, 1999

[32] K. Immink, "The Digital Versatile Disc (DVD): System Requirements and Channel Coding", SMPTE Journal, vol. 105, no. 8, Aug. 1996, p. 483-489.

[33] J. Bergmans, Digital Baseband Transmission and Recording, Kluwer Academic Publishers, 1996.

[34] ISO/IEC IS 13818, "Information Technology - Generic Coding of Moving Pictures and Associated Audio Information, Part 1: Systems," Nov. 1994.

[35] ISO/IEC IS 13818, "Information Technology - Generic Coding of Moving Pictures and Associated Audio Information, Part 2: Video," Nov. 1994.

[36] ISO/IEC IS 13818, "Information Technology - Generic Coding of Moving Pictures and Associated Audio Information, Part 3: Audio," Nov. 1994.

[37] G. Forney, "The Viterbi Algorithm", Proceedings of the IEEE, vol 61, no. 3, March 1973.

[38] G. Clark, J. Cain, Error-Correction Coding for Digital Communications, Plenum Press, 1981.

[39] G. Feygin, "A Multiprocessor Architecture for Viterbi Decoders with Linear Speed-up", MASc. Thesis, University of Toronto, 1990.

[40] R. McEliece, I. Onyszchuk, "Truncation Effects in Viterbi Decoding", IEEE Conference on Military Communications, vol. 1, p. 541-545.

[41] C. Deltoso, M. Cand, L. Sponga, "A punctured Viterbi decoder compatible with DVB standards", Proceedings of the 22nd European Solid-State Circuits Conference (ESSCIRC '96), 1996, p. 368-371.

[42] C. Berrou, C. Douillard, "Pseudo-syndrome method for supervising Viterbi decoders at any coding rate", Electronics Letters, vol. 3, no. 13, June 1994, p. 1036-1037.

[43] S. Wicker, Error control systems for digital communication and storage, Prentice-Hall, 1995.

[44] H. Shao, T. Truong, L. Deutsch, J. Yuen, I. Reed, "A VLSI Design of a Pipeline ReedSolomon Decoder", IEEE Transactions on Computers, vol. C-34, no. 5, May 1985, p. 393 403. 
[45] H. Shao, T. Truong, I. Hsu, L. Deutsch, "Architecture for time or transform domain decoding of Reed-Solomon codes", U.S. Patent US4868828, 1989.

[46] H. Shao, I. Reed, "On the VLSI Design of a Pipeline Reed-Solomon Decoder Using Systolic Arrays", IEEE Transactions on Computers, vol. 37, no. 10, Oct. 1988

[47] S. Lin, D. Costello, Error Control Coding, Prentice-Hall, 1983.

[48] E. Soljanin, R. Urbanke, "An Efficient Architecture for Implementation of a Multiplier and Inverter in GF(2^8)", Bell-Labs Technical Memo, BL01 1217-960308-08TM.

[49] H. Chang, C. Shung, "A Reed-Solomon Product-Code (RS-PC) Decoder for DVD Applications", IEEE International Solid-State Circuits Conference (ISSCC), 1998, p. 390391.

[50] M. Vaupel, U. Lambrette, H. Dawid, “An All-Digital Single-Chip Symbol Synchronizer and Channel Decoder for DVB", 1997 Intermational Conference on VLSI, p. 79-90.

[51] T. Kamada, T. Fukuoka, Y. Nakai, "An Area Effective Standard Cell Based Channel Decoder LSI for Digital Satellite TV Broadcasting", VLSI Signal Processing, IX, 1996, p.337-346

[52] Motorola Semiconductor Products Sector, Asia Pacific, "MC92300, Viterbi Decoder for Digital TV", http://www.apspg.com/products/av/fec/92300.html, 1999.

[53] Comstream Corporation, "Comstream Digital TV Set-Top Receiver" (data sheet), http:// www.comstream.com/, 1998

[54] L. Christopher, J. Stewart, K. Ramaswamy, et al., "A Fully Integrated Digital Demodulation and Forward Error Correction IC for Digital Satellite Television", IEEE Custom Integrated Circuits Conference, 1995, p. $281-284$.

[55] TDA8083 Data Sheet, "Satellite Demodulator and Decoder", Philips Semiconductors, 1999.

[56] TDA8042M Data Sheet, “Quadrature Demodulator”, Philips Semiconductors, 1997.

[57] SAA7207H Data Sheet, "Reed Solomon Decoder IC", Philips Semiconductors, 1996.

[58] J. Hagenauer, P. Hoeher, "A Viterbi Algorithm with Soft-Decision Outputs and its Applications", IEEE Global Telecommunications Conference and Exhibition, 1989, vol. 3, p.1680-6.

[59] T. Schaub, J. Modestino, "An Erasure Declaring Viterbi Decoder and its Applications to Concatenated Coding Systems", IEEE International Conference on Communications, 1986, vol. 3, p.1612-16.

[60] E. Paaske, "Improved Decoding for a Concatenated Coding System Recommended by 
CCSDS", IEEE Transactions on Communications, vol. 38, no. 8, Aug. 1990, p. 1138-1144.

[61] F. Burkert, J. Hagenauer, "Improving Channel Coding of the ETSI- and MPEG-Satellite Transmission Standards", IEEE Global Telecommunications Conference, 1997, vol. 3, p. 1539-42. 
Appendix A

DVB-T Specification 
This Appendix includes an example of the inner bit interleaver, an example of the 16-QAM constellation, and the OFDM frame structure used in the DVB-T specification [2].

The "inner" bit-interleaver of Fig. 2.12 is shown in Fig. A.1.

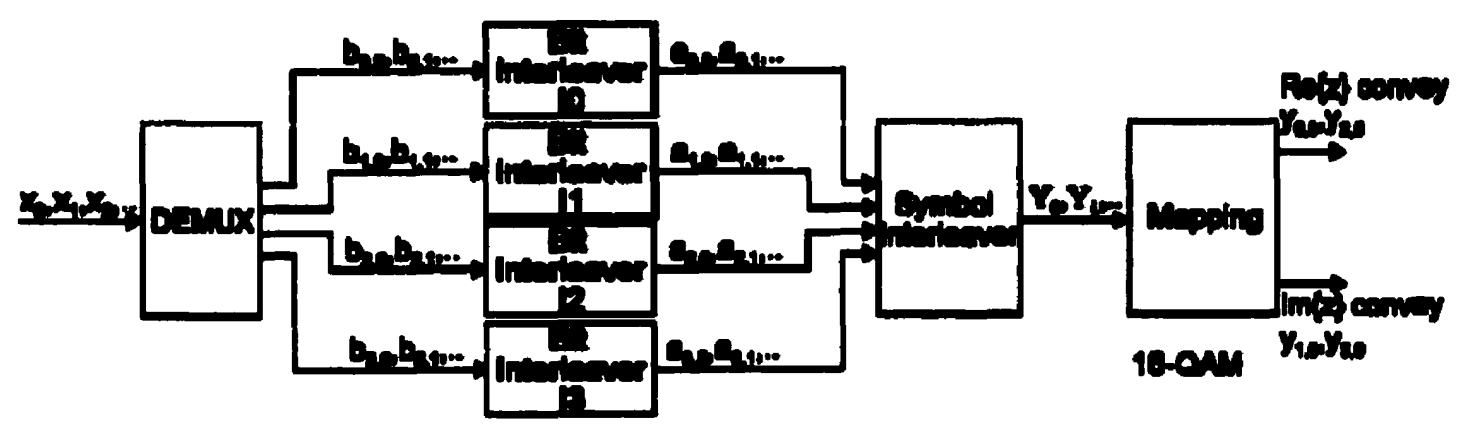

Fig. A.1: 16-QAM inner interleaver [2]

In Fig. A.1, the input of the interleaver is $x_{j}$, where $j$ is the time index of the input bit. The output of the demultiplexer is of the form $b_{i j}$, where $i$ is the stream number and $j$ is the bit number within a given stream. The demultiplexer basically sends the input bits to every other bit interleaver. That is, $x_{0}$ maps to $b_{00}, x_{1}$ maps to $b_{20}, x_{2}$ maps to $b_{10}$, and $x_{30}$ maps to $b_{30}$. Each bit-interleaver operates according to a different permutation function defined in [2], as does the symbol interleaver. The symbol interleaver combines the bit-interleaver outputs into modulation symbols $\mathbf{Y}_{\mathbf{j}}$, where $\mathrm{j}$ is the symbol number. The 16-QAM mapping operation shown in Fig. A.l illustrates the mapping of the 4-bit symbol $Y_{0}$ (composed of bits $y_{0,0}, y_{1,0}, y_{2,0}, y_{3,0}$ ) into two streams, alternately (i.e., $\left(y_{0,0}, y_{2,0}\right)$ and $\left.\left(y_{1,0}, y_{3,0}\right)\right)$.

The uniform mapping of 4-bit vectors for non-hierarchical 16-QAM is given in Fig. A.2. The 16QAM symbol $z$ is represented by two pairs of bits: the first pair denoted $\operatorname{Re}\{z\}$ determines the quadrant of the constellation the symbol is located in, and the second pair determines the position within the quadrant. The inner interleavers and constellations for hierarchical transmission are provided in [2]. 


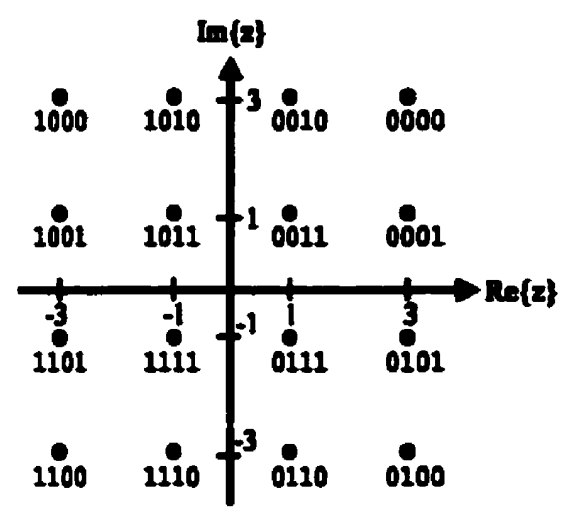

Fig. A.2: Uniform 16-QAM constellation [2]

To explain the remaining transmitter operations (i.e., OFDM), some further DVB-T system information must be introduced. DVB-T signals can be broadcast by a single terrestrial transmitter, but a single-frequency network (SFN) can be used if greater coverage area is required. An SFN is a network of transmitters, all of which send the identical signal. Since each carrier transmits at a low data rate, they have a higher immunity to multi-path interference. Multi-path signals and signals from different transmitters add constructively, as long as the echo delay is not too high. For SFNs, this puts a constraint on how far apart the transmitters can be.

There are two modes of OFDM operation defined for DVB-T, the " $2 \mathrm{~K}$ mode" and "8K mode". The 2K mode uses 1705 carriers and is used for single transmitter operation and small SFN's. The 8K mode uses 6817 carriers and can be used for large SFN networks (on a country-wide scale) [23].

Fig. A.3 shows a representation of the OFDM signal. Each row represents an OFDM symbol. Every symbol is comprised of either 1705 carriers (2K mode) or 6817 carriers (8K mode). Symbols can be considered to be split into cells, where each cell corresponds to one modulated carrier during one symbol period (illustrated as circles in Fig. A.3). Each OFDM symbol is composed of two parts: a useful part with duration $T_{U}$ and a guard interval with duration $\Delta$. The guard interval can be selected between $T_{U} / 4, T_{U} / 8, T_{U} / 16, T_{U} / 32$ based on the required level of multi-path immunity. The guard interval must be selected such that the longest path delay is less 
than the guard interval length. (In SFNs, selection of the guard interval is based on the transmitter separation.) The transmitted signal is organized in frames of 68 OFDM symbols each.

Along with the transmitted data, each OFDM frame contains scattered pilot cells, continual pilot carriers and Transmission Parameter Signalling (TPS) carriers. These are inserted in the frame adaptation block in Fig. 2.12. The pilots are transmitted at $16 / 9$ times the power level used for data. The continual pilots are mainly used for frame synchronization, and time/frequency synchronization. Scattered pilots are used for channel estimation. Scattered pilots are shown as shaded cells and continual pilots are shown as columns of shaded cells in Fig. A.3. The DVB-T standard lists the specific cells to use for scattered pilots, and the frequencies to use for continuous/TPS carriers. As the DVB-T standard provides much flexibility in transmission parameters, TPS carriers are used to signal parameters of channel coding and modulation. For example, the type of modulation being used, hierarchy information, guard interval, and inner code rates are transmitted via the TPS carriers.

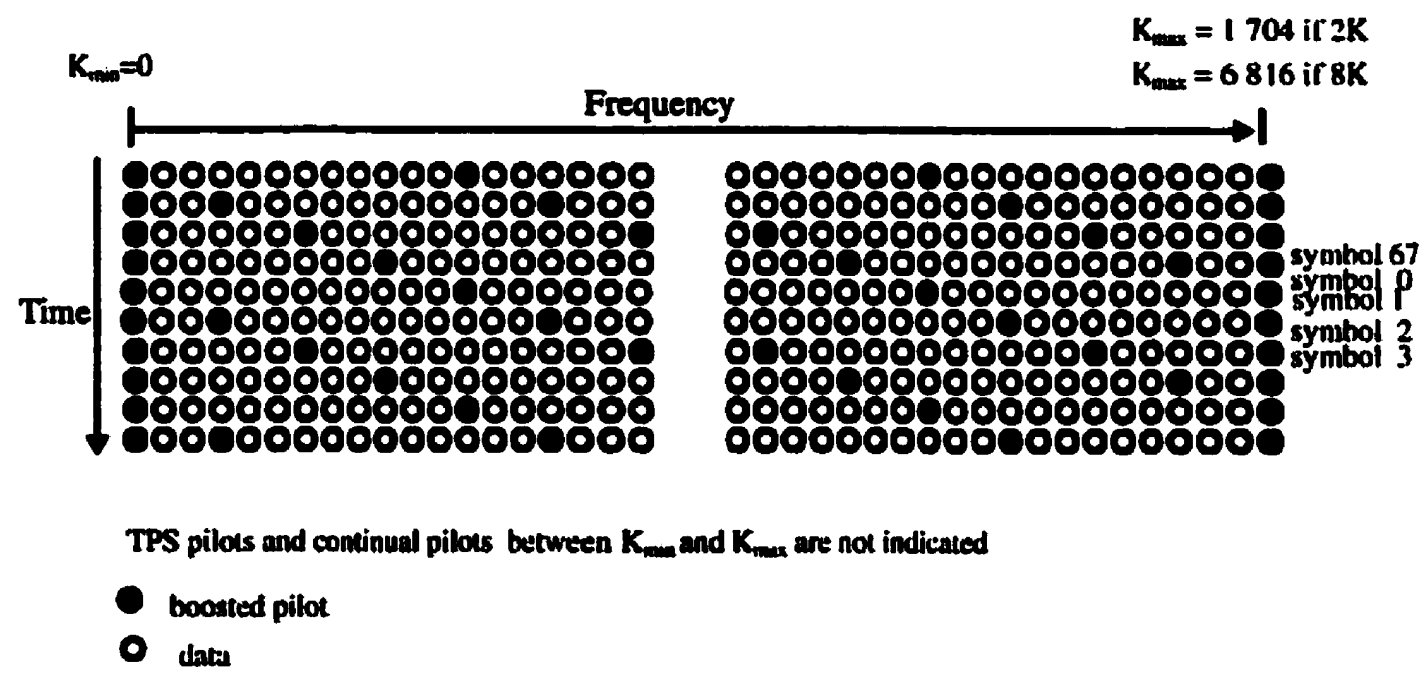

Fig. A.3: OFDM frame structure [2] 
Appendix B

Galois Field Primer 
The mathematical foundation behind Reed-Solomon codes is Galois field theory. The theory behind Galois fields is extensive and well developed. The aim here is to present the minimum amount of information on Galois field structure necessary for a basic understanding of a ReedSolomon decoder's operation. For brevity, GF properties are stated without proof. The theory and properties (including proofs) of Galois fields are explained in [43], which is the source of most of the following discussion.

To explain what a Galois Field (GF) is, we must define a group. A group is a set of objects $G$ on which the binary operation "." has been defined. When this operation is applied to any two elements of $G$, the result is another element of $G$ (closure). For $G$ to be considered a group, the operation must have the following properties:

1. Associativity: $(a \cdot b) \cdot c=a \cdot(b \cdot c)$ for all $a, b, c \in G$

2. Identity: an element $e \in G$ exists which satisfies $a \cdot e=e \cdot a=a$ for all $a \in G$.

3. Inverse: for each $a \in G$, there exists a unique element $a^{-1} \in G$ which satisfies the condition $a \cdot a^{-1}=a^{-1} \cdot a=e$.

A commutative group satisfies a fourth condition:

4. Commutativity: for all $a, b \in G, a \cdot b=b \cdot a$

For commutative groups, the group operation is considered "additive", and can be represented with the symbol " + ". The number of elements in the group is termed the "order" (or cardinality) of a group.

A field $F$ is a set of objects on which two operations + ("addition") and - ("multiplication") are defined, and the following properties are defined:

1. F forms a commutative group under + , with the additive identity element $e$ labelled " 0 ". 
2. $F$ - $\{0\}$ (the set of objects in $F$ excluding 0 ) forms a commutative group under - with the multiplicative identity labelled " 1 ".

3. The field operations distribute: $a \cdot(b+c)=(a \cdot b)+(a \cdot c)$

Fields of finite order $q$, where $q$ is the power of a prime, are called Galois fields (or finite fields) and denoted $\mathrm{GF}(q)$.

The simplest Galois field is $\mathrm{GF}(2)$, with elements $\{0,1\}$ under standard binary addition and multiplication. The operation tables for these operations are:

$$
\begin{array}{l|lll|ll}
+ & 0 & 1 & + & 0 & 1 \\
\hline 0 & 0 & 1 & 0 & 0 & 0 \\
1 & 1 & 0 & 1 & 0 & 1
\end{array}
$$

Consider $\mathrm{GF}(q)$, two elements of which are an element $\beta$ and the multiplicative identity element 1. For convenience, let the power notation $\beta^{n}$ represent $\beta$ multiplied by itself $\boldsymbol{n}$ times. Since $\beta$ is in $\mathrm{GF}(q)$, all successive powers of $\beta$ must be contained in $\mathrm{GF}(q)$ by the multiplicative closure property. The non-zero elements of $G F(q)$ have the form: $\left\{1, \beta, \beta^{2}, \beta^{3}, \beta^{4}, \ldots\right\}$ if $\beta$ is primitive. Since $\operatorname{GF}(q)$ has a finite number of elements, the sequence eventually starts to repeat values it already contains. The first value to repeat is 1 , and we define the order of a GF(q) element $\beta$ (denoted "ord $(\beta)$ ") as the smallest positive integer $m$ such that $\beta^{m}=1$. An element of order $(q-1)$ in $\mathrm{GF}(q)$ is called the primitive element in $\mathrm{GF}(q)$. All nonzero elements in $\mathrm{GF}(q)$ can be represented as $(q-1)$ consecutive powers of the primitive element $\alpha$. Two Galois field elements can be multiplied by adding their exponents modulo $(q-1)$.

A polynomial $p(x)$ of degree $m$ with coefficients taken from $\mathrm{GF}(p)$ which satisfies certain properties ${ }^{1}$ is considered "primitive". A root $\alpha$ of such a primitive polynomial has order $\left(p^{m}-1\right)$ is called a primitive element in GF( $\left(p^{m}\right)$. The $\left(p^{m}-1\right)$ consecutive powers of $\alpha$ form a 
multiplicative group of order $\left(p^{m}-1\right)$. All powers of $\alpha$ can be expressed as polynomials in $\alpha$ of degree $(m-1)$ or less (with coefficients and coefficient operations taken from $\operatorname{GF}(p)$ ) by reduction modulo the primitive polynomial. These polynomials and zero form an additive group under polynomial addition. These principles can be used to generate the non-zero elements of a Galois field. Galois fields of interest for practical RS code implementations take the form $G F\left(2^{m}\right)$, and we restrict the discussion to fields of this type.

As an example, consider the degree-4 primitive polynomial $x^{4}+x+1$ with coefficients from GF(2). Let $\alpha$ be a root of this polynomial so that $\alpha^{4}+\alpha+1=0$, or $\alpha^{4}=\alpha+1$. This relation can be used to recursively generate elements of $\mathrm{GF}\left(2^{4}\right)$ in polynomial form (degree $(4-1)$ or less) from the exponential form:

$$
\begin{aligned}
& \alpha^{0}=1 \\
& \alpha^{1} \\
& \alpha^{2} \\
& \alpha^{3} \\
& \alpha^{4}=\alpha+1 \\
& \alpha^{5}=\left(\alpha^{4}\right) \alpha=(\alpha+1) \alpha=\alpha^{2}+\alpha \\
& \alpha^{6}=\left(\alpha^{5}\right) \alpha=\left(\alpha^{2}+\alpha\right) \alpha=\alpha^{3}+\alpha^{2} \\
& \alpha^{7}=\left(\alpha^{6}\right) \alpha=\left(\alpha^{3}+\alpha^{2}\right) \alpha=\alpha^{4}+\alpha^{3}=\alpha^{3}+\alpha+1 \\
& \cdot \\
& \alpha^{14}=\alpha^{3}+1 \\
& \alpha^{15}=\left(\alpha^{14}\right) \alpha=\left(\alpha^{3}+1\right) \alpha=(\alpha+1)+\alpha=1
\end{aligned}
$$

Table 4.1 shows how the polynomial form can be represented by $m$-bit vectors. This bit-vector

1. The polynomial $p(x)$ cannot be factored into a product of lower degree polynomials with coefficients in GF(p). The smallest positive integer $n$ for which $p(x)$ divides $x^{n}-1$ must be $n=p^{n}-1$. 
form is used later in actual GF arithmetic hardware design.

Table A.1: GF( $\left.2^{4}\right)$ elements generated from $\alpha^{4}+\alpha+1=0$

\begin{tabular}{c|c|c|c|c}
\hline $\begin{array}{c}\text { Power } \\
\text { form }\end{array}$ & $\alpha^{3}$ & $\alpha^{2}$ & $\alpha^{1}$ & $\alpha^{0}$ \\
\hline$\alpha^{0}$ & 0 & 0 & 0 & 1 \\
\hline$\alpha^{1}$ & 0 & 0 & 1 & 0 \\
\hline$\alpha^{2}$ & 0 & 1 & 0 & 0 \\
\hline$\alpha^{3}$ & 1 & 0 & 0 & 0 \\
\hline$\alpha^{4}$ & 0 & 0 & 1 & 1 \\
\hline$\alpha^{5}$ & 0 & 1 & 1 & 0 \\
\hline$\alpha^{6}$ & 1 & 1 & 0 & 0 \\
\hline$\alpha^{7}$ & 1 & 0 & 1 & 1 \\
\hline$\alpha^{8}$ & 0 & 1 & 0 & 1 \\
\hline$\alpha^{9}$ & 1 & 0 & 1 & 0 \\
\hline$\alpha^{10}$ & 0 & 1 & 1 & 1 \\
\hline$\alpha^{11}$ & 1 & 1 & 1 & 0 \\
\hline$\alpha^{12}$ & 1 & 1 & 1 & 1 \\
\hline$\alpha^{13}$ & 1 & 1 & 0 & 1 \\
\hline$\alpha^{14}$ & 1 & 0 & 0 & 1 \\
\hline & & &
\end{tabular}

Note that the exponential form facilitates multiplication of GF elements (exponents are added modulo $\left(2^{4}-1\right)$ ), whereas the polynomial/bit-vector form is amenable to GF addition (bit-vectors are XORed). For example, $\alpha^{12} \cdot \alpha^{5}=\alpha^{2}$ and $\alpha^{12}+\alpha^{5}=1111+0110=1001=\alpha^{14}$.

For the RS code relevant to DVB and DVD, the primitive polynomial for $G F\left(2^{8}\right)$ is $p(x)=x^{8}+x^{4}$ $+x^{3}+x^{2}+x^{0}$, which implies $\alpha^{8}=\alpha^{4}+\alpha^{3}+\alpha^{2}+\alpha^{0}$. A similar process can be followed to generate all 256 elements of $\mathrm{GF}\left(2^{8}\right)$. Note that in the bit-vector representation of field elements, each symbol is one byte. 
Appendix C

\section{Time Domain Reed-Solomon Decoding}


This Appendix briefly describes a time-domain decoder (TDD) architecture based on the design in [45] and [46]. An advantage of the TDD over the frequency domain decoder (FDD) is that the TDD has lower area, at the expense of higher computational complexity. If a RS decoder is to be designed for mass production, the TDD would be appropriate (the FDD was used in the thesis due to its lower complexity, which saved time in design and testing). The decoder architecture is the same up to and including the Modified Euclid's Algorithm (MEA). The results of the MEA are used differently, however. The error locator $\sigma(x)$ is assigned to the MEA output $\mu_{i}(x)$, and the "error evaluator" polynomial $W(x)$ is assigned to the MEA output $R_{i}(x)$. Note that the $R_{i}(x)$ output is not used in the FDD. Fig. C.I shows the TDD architecture. Note that no delay for the syndromes are required, as in the FDD.

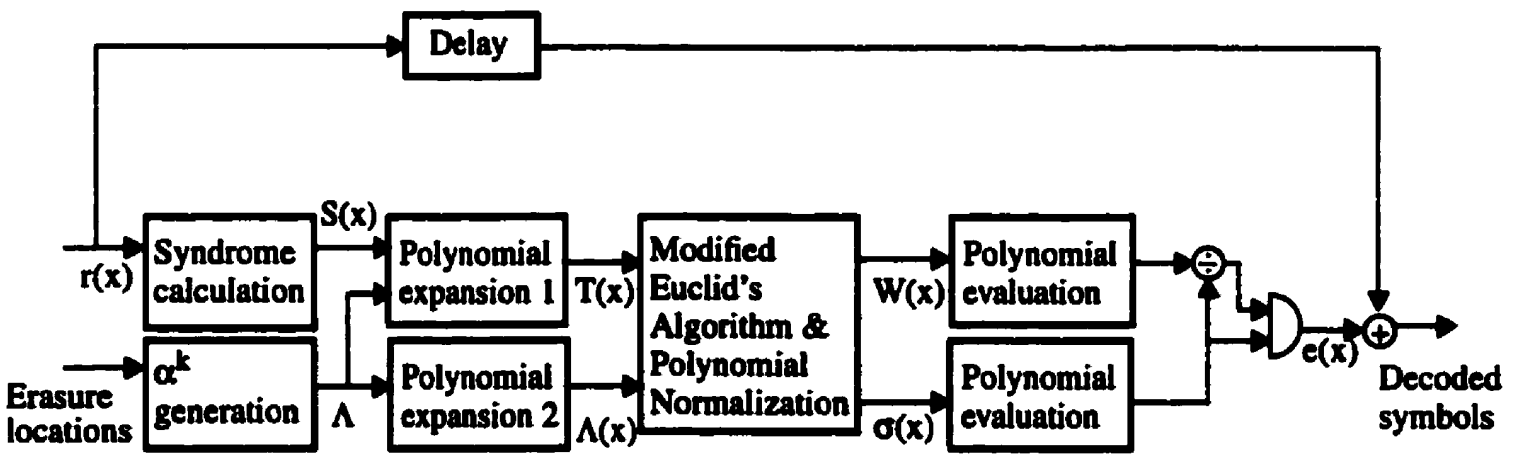

Fig. C.1: Time domain decoder structure [46]

The $\alpha^{k}$ generation and polynomial expansion blocks are given in Appendix D.

The basic idea of the TDD is to correct RS codewords by calculating the error locations and magnitudes. The error locator polynomial $\sigma(x)$ must be evaluated for $\alpha^{-i}, i=0, \ldots, N-1$ to find the roots of $\sigma(x)$. If $\sigma\left(\alpha^{-i}\right)=0, r_{i}$ is a corrupted symbol of the RS codeword.

The error magnitudes are computed by evaluating $W(x)$ and $\sigma^{\prime}(x)$ for $\alpha^{-i}, i=0, \ldots, N-1$. 
Specifically, the error magnitudes are: $\frac{W\left(\alpha^{-i}\right)}{\sigma^{\prime}\left(\alpha^{-i}\right)}, 0 \leq i \leq N-1$.

The polynomial evaluation blocks have the form of Fig. C.2, which shows the circuit for computing the error locations. For details, see [46].

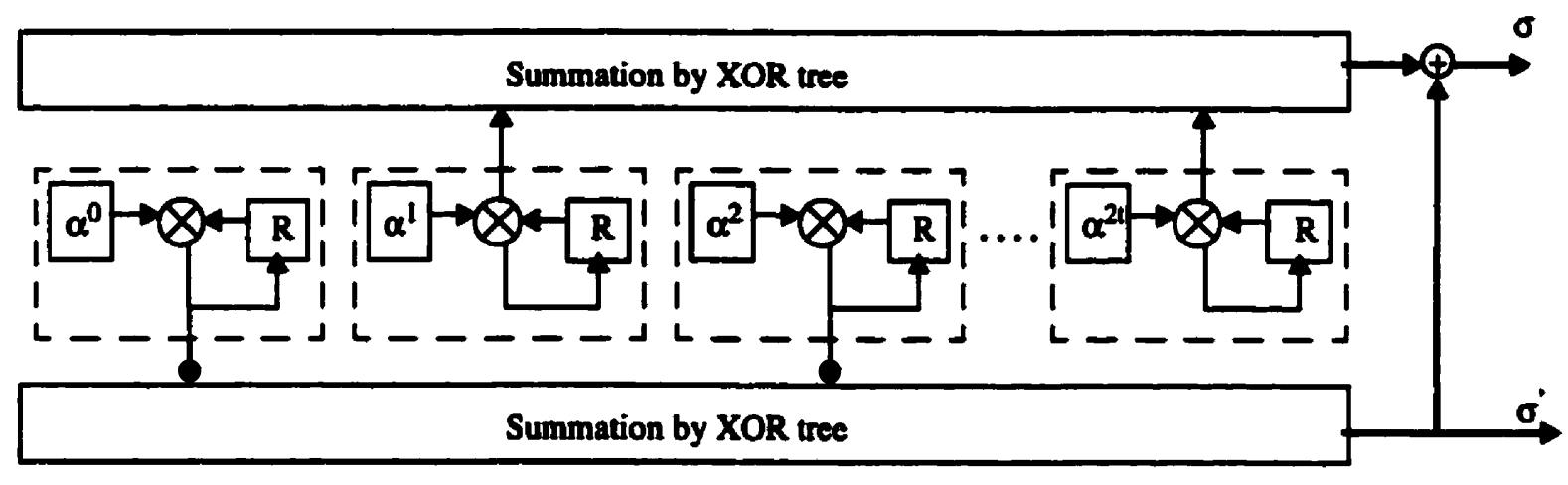

Fig. C.2: Polynomial evaluation for $\sigma$ [46] 


\section{Appendix D}

\section{Hardware for Erasure RS Decoding}


This Appendix presents the $\alpha^{k}$ generation and polynomial expansion blocks introduced in Fig. 4.7. For $\alpha^{k}$ generation, $\alpha^{0}$ could be loaded into a register, and multiplied by $\alpha$ until all values are obtained. Instead of using a general multiplier, we use a GF arithmetic technique from [47] to design a circuit which multiplies a GF element by $\alpha$. Consider a GF $\left(2^{8}\right)$ element represented as

$\beta=b_{0} \alpha^{0}+b_{1} \alpha^{1}+b_{2} \alpha^{2}+b_{3} \alpha^{3}+b_{4} \alpha^{4}+b_{5} \alpha^{5}+b_{6} \alpha^{6}+b_{7} \alpha^{7}$

where $\alpha$ is the primitive element in the field, and $b_{i}(0 \leq i \leq 7)$ are binary digits. Using the fact that $\alpha^{8}=\alpha^{4}+\alpha^{3}+\alpha^{2}+\alpha^{0}$, we can multiply the element by $\alpha$ :

$\alpha \beta=b_{7} \alpha^{0}+b_{0} \alpha^{1}+\left(b_{1}+b_{7}\right) \alpha^{2}+\left(b_{2}+b_{7}\right) \alpha^{3}+\left(b_{3}+b_{7}\right) \alpha^{4}+b_{4} \alpha^{5}+b_{5} \alpha^{6}+b_{6} \alpha^{7}$

which leads to the circuit of Fig. D.1.

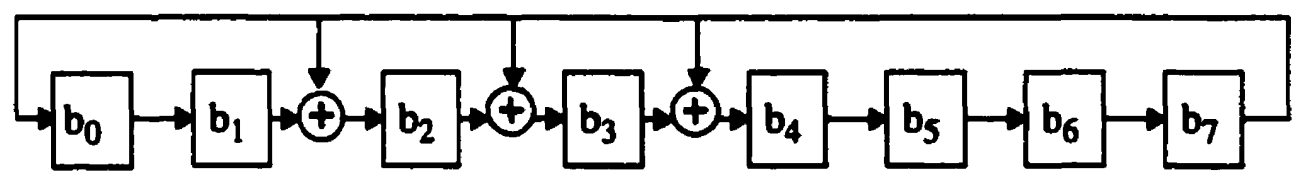

Fig. D.1: $\alpha^{k}$ generation

A counter is also be used in this unit to output the number of erasures, $v$. The number of erasures is forwarded to the MEA to determine when the error locator polynomial has been calculated.

The block labelled "Polynomial expansion 2" can be implemented as in Fig. D.2. The output of the $\alpha^{k}$ generation block are shifted in, and the switches are closed when the inputs are non-zero. 


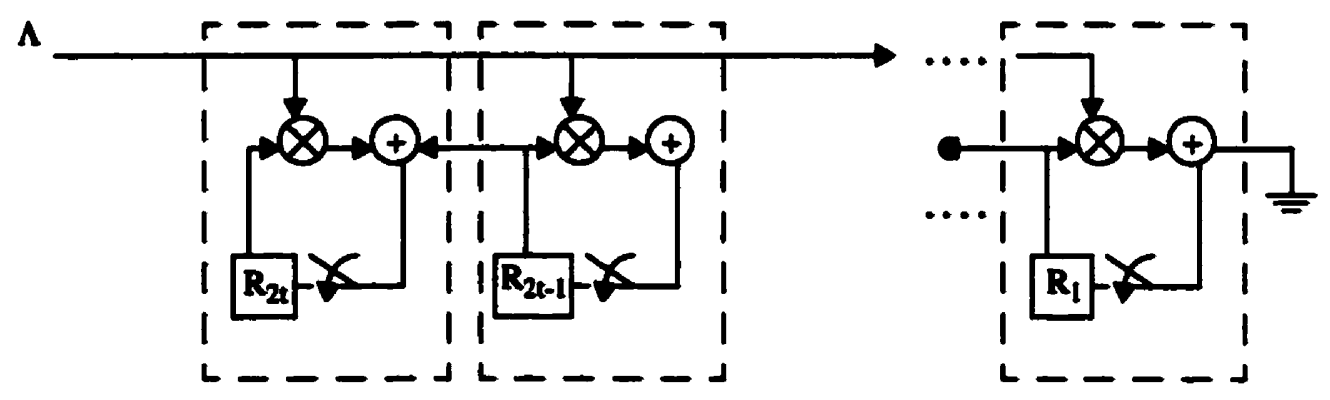

Fig. D.2: Polynomial expansion 2 [45]

The Forney syndromes are calculated by the architecture in Fig. D.3. The standard syndromes are shifted into registers $S_{k}, 1 \leq k \leq 2 t$. The output of the $\alpha^{k}$ generation block are shifted in, and the switches are closed when the inputs are non-zero; otherwise the switches are open.

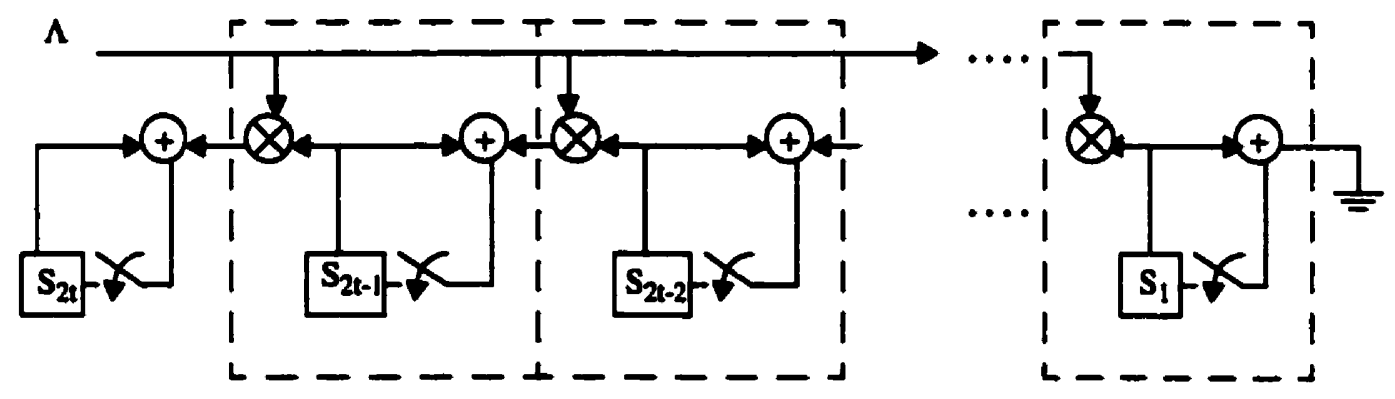

Fig. D.3: Polynomial expansion 1 [45] 
Appendix E

\section{Optimizing the ACS Arithmetic Units}


The exact way in which a design is presented to a synthesis tool greatly affects the synthesized result. Compiling a design in slightly different ways can have non-intuitive effects on the resulting synthesized circuits. Despite providing very high speed constraints, the ACS unit could only be synthesized to run at $62.5 \mathrm{Mbps}$ for the fabricated design. A different synthesis strategy was subsequently found which resulted in an ACS unit which could operate above $140 \mathrm{Mbps}$. This Appendix illustrates this ACS optimization.

The critical path of the VD is in the ACS unit, specifically in the ACS add and subtract units. For the fabricated design, the entire ACS unit was synthesized (all 64 cells) in one compilation, using a set of aggressive synthesis constraints. The synthesis tool did not meet these constraints, limiting the maximum output bit-rate of the implemented VD. Fig. E.1 shows the adder synthesized in each ACS cell, with a ripple-carry delay.

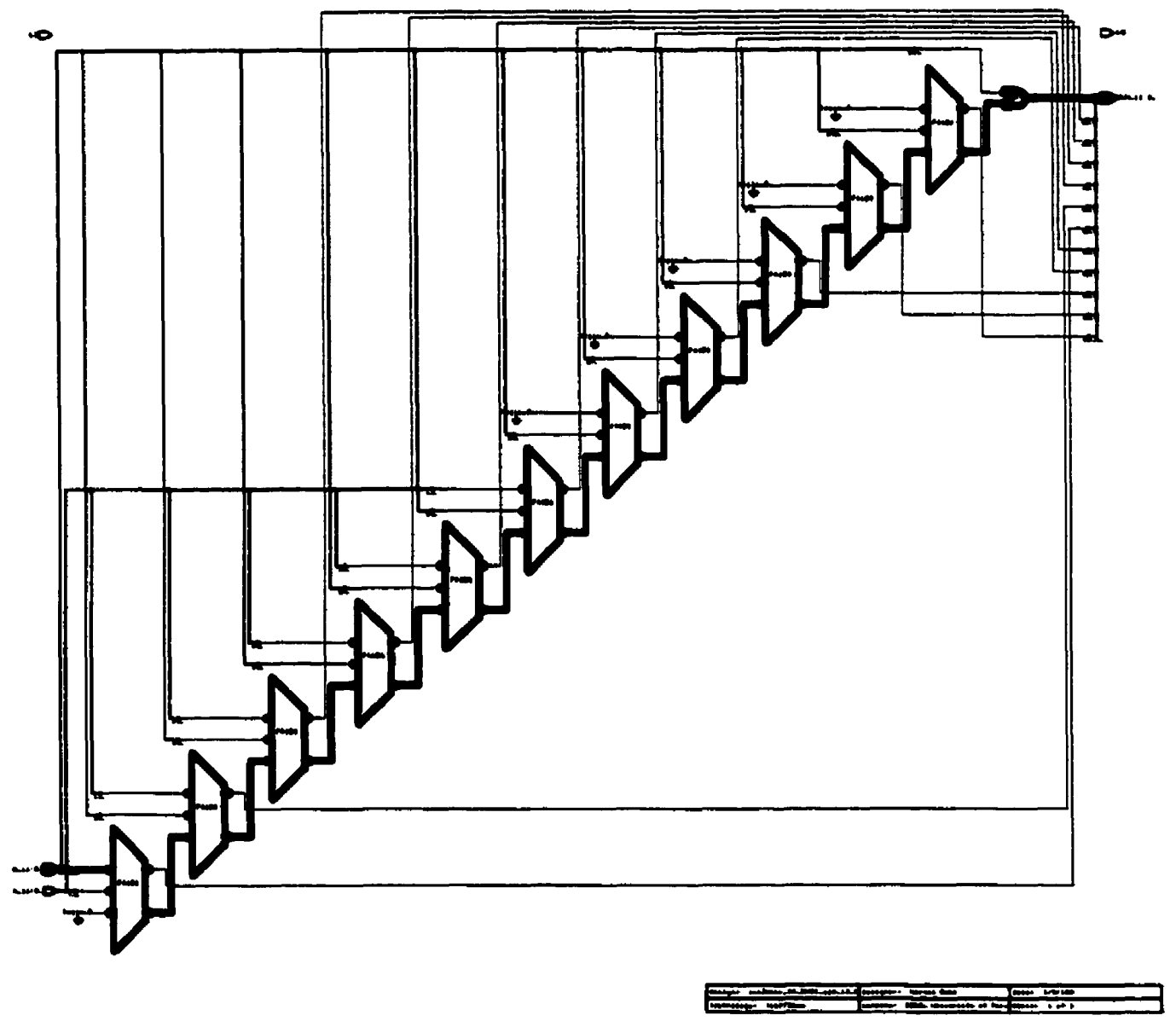

Fig. E.1: 12-bit ACS adder with ripple-carry chain as part of $16 \mathrm{~ns}$ critical path 
The other adder and subtractor in each ACS cell have a similar structure.

Although the VD was implemented with the above structure, it is possible to increase its bit throughput rate. Fig. E.2 shows the results of synthesizing a single ACS cell using the same synthesis constraints as used above. Despite the fact that the same target speed was specified in the synthesis constraints, the ACS arithmetic unit speed is much higher when a single ACS cell is synthesized. The use of this ACS cell would enable the VD to operate at a speed of $140 \mathrm{Mbps}$.

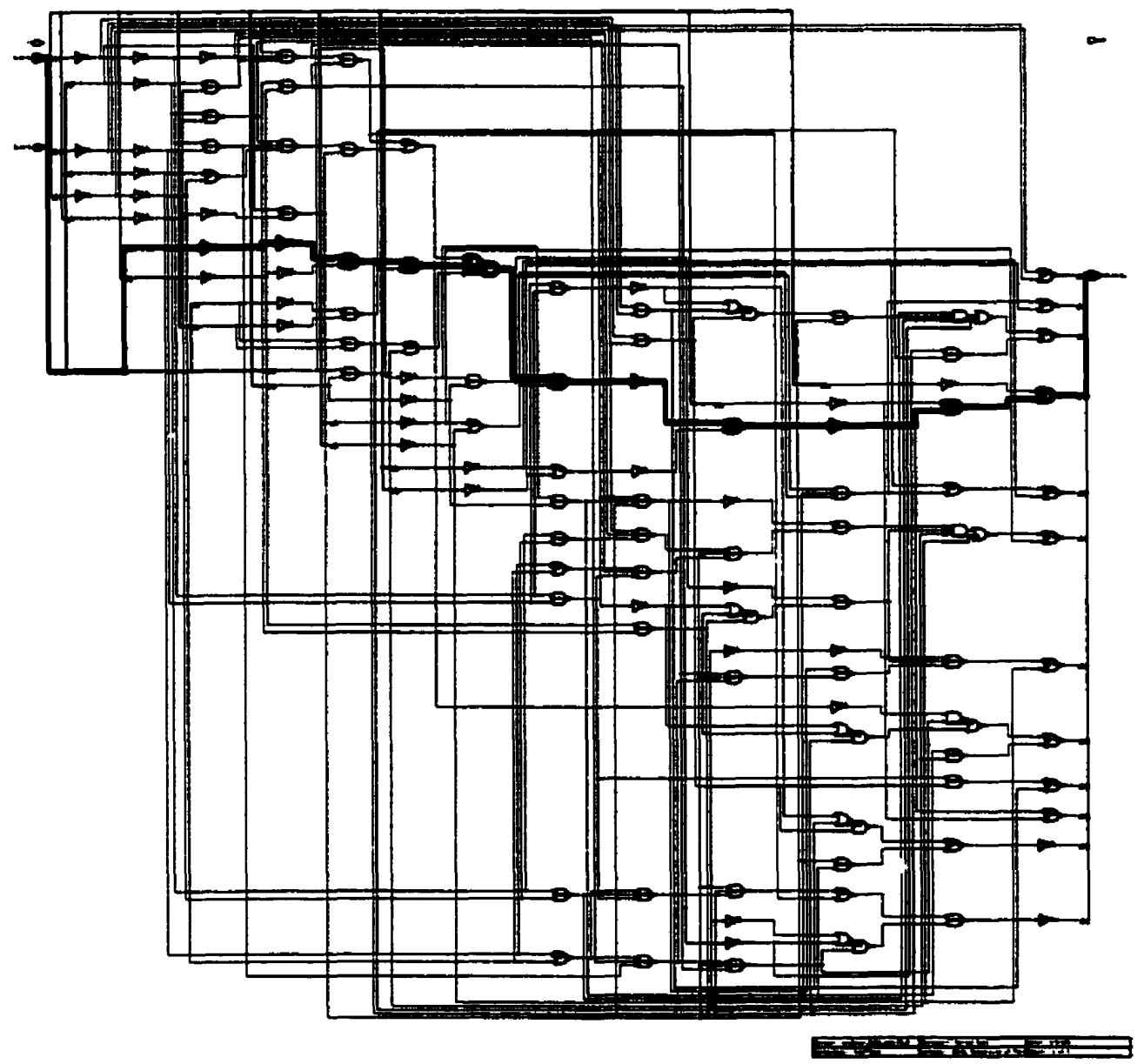

Fig. E.2: 12-bit ACS adder with critical path reduced to $\sim 7 \mathrm{~ns}$ 